Supporting Information

\title{
Controlling Sugar Deoxygenation Products from Biomass by Choice of Fluoroarylborane Catalyst
}

Youngran Seo, Jared M. Lowe and Michel R. Gagné*

Department of Chemistry, The University of North Carolina at Chapel Hill, Chapel Hill, North Carolina 27599, United States

*mgagne@unc.edu

\section{Table of Contents}

$\begin{array}{ll}\text { General Experimental } & \text { S2 }\end{array}$

$\begin{array}{ll}\text { Experimental procedures } & \text { S3 }\end{array}$

$\begin{array}{ll}\text { References for experimental analysis } & \text { S12 }\end{array}$

$\begin{array}{ll}\text { Supplementary figures } & \text { S14 }\end{array}$

${ }^{1} \mathrm{H}$ and ${ }^{13} \mathrm{C}$ NMR Spectra $\quad$ S18

$\begin{array}{ll}\text { Computational details } & \text { S48 }\end{array}$

$\begin{array}{ll}\text { References for computational analysis } & \text { S48 }\end{array}$

$\begin{array}{ll}\text { Optimized coordinates } & \text { S49 }\end{array}$ 


\section{General Experimental}

General Methods: All catalytic reactions were performed in oven $\left(130^{\circ} \mathrm{C}\right)$ and/or flame dried glassware in a nitrogen-filled glovebox. All reactions were performed at ambient temperature (25 ${ }^{\circ} \mathrm{C}, \mathrm{RT}$ ) unless otherwise specified. All workup procedures were performed under air with reagent grade reagents unless otherwise specified. Column chromatography was performed using SilaFlash P60 40-63 $\mu \mathrm{m}$ (230-400 mesh). Thin layer chromatography (TLC) was performed on SiliCycle Silica Gel 60 F254 plates and was visualized with cerium ammonium molybdate (CAM) stain. The numbering and naming used in the article and SI is based on the parent sugar.

All NMR spectra were recorded on a Bruker Avance $600 \mathrm{MHz}$ spectrometer at standard temperature and pressure. All deuterated solvents were used as received from Cambridge Isotope Laboratories, Inc. The residual solvent protons $\left({ }^{1} \mathrm{H}\right)$ or the solvent carbons $\left({ }^{13} \mathrm{C}\right)$ were used as internal standards. ${ }^{2}$ The following abbreviations are used in reporting NMR data: s, singlet; $d$, doublet; t, triplet; dd, doublet of doublets; dt, doublet of triplets; dq, doublet of quartets; td, triplet of doublets; ddd, doublet of doublet of doublets; and m, multiplet. Where necessary, 2D COSY, and HSQC data were used for peak assignment. High Resolution Mass spectra were obtained on Q Exactive ${ }^{\mathrm{TM}}$ HF-X Hybrid Quadrupole-Orbitrap ${ }^{\mathrm{TM}}$ Mass spectrometer. Optical rotation values were measured with a Rudolph Research Analytical (A21102. API/1W) polarimeter.

\section{Chemicals:}

All chemicals were used as received, or otherwise described on how it was treated before use. 2Deoxy-D-glucose, 2-deoxy-D-galactose, D-xylose, and 1-OMe-D-galactose were purchased form

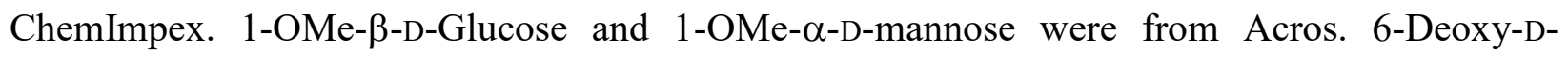
glucose and 6-deoxy-L-galacotse (L-fucose) were purchased from Carbosynth, and 1-OMe- $\beta$-Dxylose was purchased from TCI. $\mathrm{Me}_{2} \mathrm{EtSiH}$ and $\mathrm{Et}_{3} \mathrm{SiH}$ were purchased from Gelest, and degassed via three freeze-pump-thaw cycles and stored over molecular sieves in the glovebox. Pyridine, triethylamine, methanol and dichloromethane $\left(\mathrm{CH}_{2} \mathrm{Cl}_{2}\right)$ were purchased from Fisher. $\mathrm{CH}_{2} \mathrm{Cl}_{2}$ was passed through an alumina column in a solvent purification system and degassed via three freeze-

pump-thaw cycles and stored over molecular sieves in the glovebox prior to use.

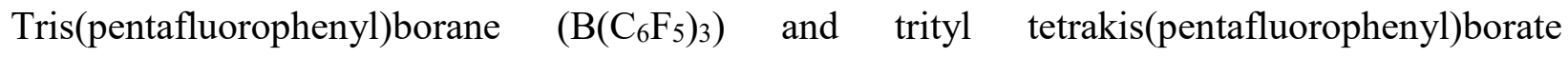
$\left(\left[\mathrm{Ph}_{3} \mathrm{C}\right]\left[\mathrm{B}\left(\mathrm{C}_{6} \mathrm{~F}_{5}\right)_{4}\right]\right)$ were purchased from Strem and used as received. BAr ${ }_{3,5-\mathrm{CF} 3}$ was synthesized 
by following the previous method. ${ }^{1}$ All per-silylated substrates were prepared by following the protocol delineated in previous publications. ${ }^{2,3}$

General procedure for reduction of simple ethers: In a $\mathrm{N}_{2}$-filled glove box, BAr, 3 -CF3 $(3.63 \mathrm{mg}$, $0.006 \mathrm{mmol}, 0.05$ equiv) was placed in a 1 dram vial and dissolved in $0.4 \mathrm{~mL}$ of $\mathrm{CH}_{2} \mathrm{Cl}_{2}$. To the catalyst solution, TESH ( $27 \mu \mathrm{L}, 0.168 \mathrm{mmol}, 1.50$ equiv) was added and mixed until $\mathrm{BAr}_{3,5-\mathrm{CF} 3}$ dissolved. In a separate vial, isochroman (15 mg, $0.112 \mathrm{mmol}, 1.00$ equiv) was diluted with 0.1 $\mathrm{mL}$ of $\mathrm{CH}_{2} \mathrm{Cl}_{2}$. The catalyst and silane mixture was then added to the substrate solution in one portion. The reaction mixture was transferred to an NMR tube and sealed with a septum cap. Then the reaction mixture was monitored by ${ }^{13} \mathrm{C}$ NMR. The in situ monitored spectra are shown in Figure S1 and S2.

General procedure for deoxygenation of biomass derived sugars: In a $\mathrm{N}_{2}$-filled glove box, $\mathrm{BAr}_{3,5-\mathrm{CF} 3}(5.23 \mathrm{mg}, 0.008 \mathrm{mmol}, 0.10$ equiv) was placed in a 1 dram vial and dissolved in $0.4 \mathrm{~mL}$ of $\mathrm{CH}_{2} \mathrm{Cl}_{2}$. To the catalyst solution, was added TESH $(19 \mu \mathrm{L}, 0.121 \mathrm{mmol}, 1.50$ equiv $)$ and mixed. In a separate vial, TES-2-deoxyglucose $(50 \mathrm{mg}, 0.081 \mathrm{mmol}, 1.00$ equiv) was diluted with $0.1 \mathrm{~mL}$ of $\mathrm{CH}_{2} \mathrm{Cl}_{2}$. The catalyst and silane mixture was then added to the substrate solution in one portion. The reaction mixture was transferred to NMR tube and sealed with a septum cap. Once the starting material was consumed, the mixture was transferred to a vial and rinsed three times with $0.5 \mathrm{~mL}$ of $\mathrm{MeOH}$. After concentrating the resulting solution in vacuo, the crude residue was purified by silica gel chromatography $\left(20: 1 \mathrm{CH}_{2} \mathrm{Cl}_{2} / \mathrm{MeOH}\right.$ to $10: 1$ to $\left.5: 1\right)$ to yield 1,2-deoxyglucose (1) as a clear film in 92\% yield (11.0 mg, average over two runs).

For $\mathrm{B}\left(\mathrm{C}_{6} \mathrm{~F}_{5}\right)_{3}$ catalyzed conditions, $\mathrm{B}\left(\mathrm{C}_{6} \mathrm{~F}_{5}\right)_{3}(4.12 \mathrm{mg}, 0.008 \mathrm{mmol}, 0.10$ equiv. $)$ was used instead of $\mathrm{BAr}_{3,5-\mathrm{CF} 3}$ and followed the same procedure as described above. 
General procedure for 1 g-scale reactions: In a $\mathrm{N}_{2}$-filled glove box, $\mathrm{B}\left(\mathrm{C}_{6} \mathrm{~F}_{5}\right)_{3}(41.2 \mathrm{mg}, 0.08$ mmol, 0.05 equiv) was placed in a $20 \mathrm{~mL}$ vial and dissolved in $3.0 \mathrm{~mL}$ of $\mathrm{CH}_{2} \mathrm{Cl}_{2}$. To the catalyst solution, was added TESH (643 $\mu \mathrm{L}, 4.02 \mathrm{mmol}, 2.50$ equiv) and mixed. In a separate $20 \mathrm{~mL}$ vial, TES-2-deoxyglucose (1.0 g, $1.610 \mathrm{mmol}, 1.00$ equiv) was diluted with $8.0 \mathrm{~mL}$ of $\mathrm{CH}_{2} \mathrm{Cl}_{2}$. The catalyst and silane mixture was then added slowly to the substrate solution. The reaction mixture was stirred in the glove box until the gas evolution stopped, and then it was sealed and reacted at room temperature in the glove box. After $45 \mathrm{~h}$, the reaction mixture was transferred to $100 \mathrm{~mL}$ round bottom flask and $15 \mathrm{~mL}$ of $\mathrm{MeOH}$ was added. Then, the resulting solution was concentrated under reduced pressure, and the crude residue was purified by silica gel chromatography (20:1 $\mathrm{CH}_{2} \mathrm{Cl}_{2} / \mathrm{MeOH}$ to $10: 1$ to $\left.6: 1\right)$ to yield 1,2-deoxysorbitol (3) as a white power in $91 \%$ yield (221.0 $\mathrm{mg}$ ).

For $\mathrm{BAr}_{3,5-\mathrm{CF} 3}$ catalyzed conditions, $\mathrm{BAr}_{3,5-\mathrm{CF} 3}(52.3 \mathrm{mg}, 0.080 \mathrm{mmol}, 0.05$ equiv) was used instead of $\mathrm{B}\left(\mathrm{C}_{6} \mathrm{~F}_{5}\right)_{3}$ and followed the same procedure as described above, except that the $\mathrm{BAr} 3,5-\mathrm{CF} 3$ solution was stirred until fully dissolved before it was added to the substrate solution. After the reaction and purification, 1,2-deoxyglucose was obtained in 79\% yield (188 mg).

\section{Procedure for preparing 16 and Si-16 to measure optical purity}

Condition A: In a $\mathrm{N}_{2}$-filled glove box, $\mathrm{B}\left(\mathrm{C}_{6} \mathrm{~F}_{5}\right)_{3}(2.52 \mathrm{mg}, 0.005 \mathrm{mmol}, 0.05$ equiv) was placed in a 1 dram vial and dissolved in $0.4 \mathrm{~mL}$ of $\mathrm{CH}_{2} \mathrm{Cl}_{2}$. To the catalyst solution, TESH $(55 \mu \mathrm{L}, 0.345$ mmol, 3.50 equiv) was added and mixed. In a separate vial, TES-1-OMe-xylose (50 mg, 0.099 mmol, 1.00 equiv) was diluted with $0.1 \mathrm{~mL}$ of $\mathrm{CH}_{2} \mathrm{Cl}_{2}$. The catalyst and silane mixture was then added to the substrate solution in one portion. The reaction mixture was transferred to an NMR 
tube and sealed with a septum cap. After $24 \mathrm{~h}$, the mixture was transferred to a vial and rinsed three times with $0.5 \mathrm{~mL}$ of $\mathrm{MeOH}$. After concentrating the resulting solution in vacuo, the crude residue was purified by silica gel chromatography $\left(20: 1 \mathrm{CH}_{2} \mathrm{Cl}_{2} / \mathrm{MeOH}\right.$ to $10: 1$ to $\left.5: 1\right)$ to yield 1deoxy-xylitol (16) as a clear film in $82 \%$ yield $(11.0 \mathrm{mg})$.

To obtain Si-16 under condition A, the reaction mixture was quenched with TEA $(50 \mu \mathrm{L})$ and then transferred to a vial and rinsed three times with $0.5 \mathrm{~mL}$ of $\mathrm{CH}_{2} \mathrm{Cl}_{2}$. After concentrating the solution under reduced pressure, the crude was purified by silica gel chromatography (50:1 Hexane:ether to $20: 1$ to $10: 1$ ) to yield per-TES-1-deoxy-xylitol (Si-16) as a colorless liquid in 75\% yield (44 $\mathrm{mg})$.

Condition B: In a $\mathrm{N}_{2}$-filled glove box, $\mathrm{BAr}_{3,5-\mathrm{CF} 3}(3.21 \mathrm{mg}, 0.005 \mathrm{mmol}, 0.05$ equiv) was placed in a 1 dram vial and dissolved in $0.4 \mathrm{~mL}$ of $\mathrm{CH}_{2} \mathrm{Cl}_{2}$. To the catalyst solution, TESH $(55 \mu \mathrm{L}, 0.345$ mmol, 3.50 equiv) was added and mixed until $\mathrm{BAr}_{3,5-\mathrm{CF} 3}$ dissolved. In a separate vial, TES-1-OMexylose (50 mg, $0.099 \mathrm{mmol}, 1.00$ equiv) was diluted with $0.1 \mathrm{~mL}$ of $\mathrm{CH}_{2} \mathrm{Cl}_{2}$. The catalyst and silane mixture was then added to the substrate solution in one portion. The reaction mixture was transferred to an NMR tube and sealed with a septum cap. After $24 \mathrm{~h}$, the reaction mixture was quenched with TEA $(50 \mu \mathrm{L})$ and then transferred to a vial and rinsed three times with $0.5 \mathrm{~mL}$ of $\mathrm{CH}_{2} \mathrm{Cl}_{2}$. After concentrating the solution under reduced pressure, the crude was purified by silica gel chromatography (50:1 Hexane:ether to 20:1 to 10:1) to yield per-TES-1,4-anhydroxylitol (Si14) as a colorless liquid in $79 \%$ yield (37 mg). Si-14 was taken into a glove box and used for the subsequent step. In a $\mathrm{N}_{2}$-filled glove box, $\mathrm{B}\left(\mathrm{C}_{6} \mathrm{~F}_{5}\right)_{3}(1.99 \mathrm{mg}, 0.004 \mathrm{mmol}, 0.05$ equiv) was placed in a 1 dram vial and dissolved in $0.4 \mathrm{~mL}$ of $\mathrm{CH}_{2} \mathrm{Cl}_{2}$. To the catalyst solution, TESH $(14 \mu \mathrm{L}, 0.085$ mmol, 1.10 equiv) was added and mixed. In a separate vial, Si-14 (37 mg, $0.078 \mathrm{mmol}, 1.00$ equiv) 
was diluted with $0.1 \mathrm{~mL}$ of $\mathrm{CH}_{2} \mathrm{Cl}_{2}$. The catalyst and silane mixture was then added to the substrate solution in one portion. The reaction mixture was transferred to NMR tube and sealed with a septum cap. After $18 \mathrm{~h}$, the mixture was transferred to a vial and rinsed three times with $0.5 \mathrm{~mL}$ of $\mathrm{MeOH}$. After concentrating the resulting solution in vacuo, the crude residue was purified by silica gel chromatography $\left(20: 1 \mathrm{CH}_{2} \mathrm{Cl}_{2} / \mathrm{MeOH}\right.$ to $10: 1$ to 5:1) to yield 1-deoxy-xylitol (16) as a clear film in $95 \%$ yield $(10.0 \mathrm{mg})$.

To obtain Si-16 under condition B, in a $\mathrm{N}_{2}$-filled glove box, BAr 3,5 -CF3 $(3.21 \mathrm{mg}, 0.005 \mathrm{mmol}, 0.05$ equiv) was placed in a 1 dram vial and dissolved in $0.4 \mathrm{~mL}$ of $\mathrm{CH}_{2} \mathrm{Cl}_{2}$. To the catalyst solution, TESH (55 $\mu \mathrm{L}, 0.345 \mathrm{mmol}, 3.50$ equiv) was added and mixed until $\mathrm{BAr}_{3,5-\mathrm{CF} 3}$ dissolved. In a separate vial, TES-1-OMe-xylose (50 mg, $0.099 \mathrm{mmol}, 1.00$ equiv) was diluted with $0.1 \mathrm{~mL}$ of $\mathrm{CH}_{2} \mathrm{Cl}_{2}$. The catalyst and silane mixture was then added to the substrate solution in one portion. The reaction mixture was transferred to an NMR tube and sealed with a septum cap. After $24 \mathrm{~h}$, $\mathrm{B}\left(\mathrm{C}_{6} \mathrm{~F}_{5}\right)_{3}$ in $0.15 \mathrm{~mL}$ of $\mathrm{CH}_{2} \mathrm{Cl}_{2}$ was added under $\mathrm{N}_{2}$ atmosphere, then the reaction mixture reacted another $24 \mathrm{~h}$ at room temperature. The reaction mixture was quenched with TEA $(50 \mu \mathrm{L})$ and then transferred to a vial and rinsed three times with $0.5 \mathrm{~mL}$ of $\mathrm{CH}_{2} \mathrm{Cl}_{2}$. After concentrating the solution under reduced pressure, the crude was purified by silica gel chromatography (50:1 Hexane:ether to $20: 1$ to $10: 1)$ to yield per-TES-1-deoxy-xylitol (Si-16) as a colorless liquid in $86 \%$ yield (48 $\mathrm{mg})$.

Procedure for 18 under non-reductive conditions: In a $\mathrm{N}_{2}$-filled glove box, TMS-1deoxygalactose (60 mg, $0.133 \mathrm{mmol}, 1.00$ equiv) and $\left[\mathrm{Ph}_{3} \mathrm{C}\right]\left[\mathrm{B}\left(\mathrm{C}_{6} \mathrm{~F}_{5}\right)_{4}\right](24.4 \mathrm{mg}, 0.026 \mathrm{mmol}$, 0.20 equiv) were weighed into a 1 dram vial and diluted with $0.5 \mathrm{~mL}$ of $\mathrm{CH}_{2} \mathrm{Cl}_{2}$. The vial was sealed with a septum cap, and shaken several times to dissolve. To the solution, $\mathrm{Me}_{2} \mathrm{EtSiH}$ (4.4 $\mu \mathrm{L}, 0.033 \mathrm{mmol}, 0.25$ equiv) was added. The reaction mixture was then transferred into an NMR 
tube and sealed with a septum cap. Once the starting material was consumed, the mixture was quenched with TEA $(50 \mu \mathrm{L})$ and transferred to a vial with rinsing three times with $0.5 \mathrm{~mL}$ of $\mathrm{CH}_{2} \mathrm{Cl}_{2}$. After concentrating under reduced pressure, the crude residue was purified by silica gel chromatography (50:1 Hexane:ether to $20: 1$ to $10: 1$ to 5:1) to yield TMS-1-deoxy-3,6anhydrogalactose $(\mathbf{1 8}, 31 \mathrm{mg}, 81 \%)$.

In addition to the line list data that follows, our group has created a database of high-resolution ${ }^{1} \mathrm{H}$ and ${ }^{13} \mathrm{C}$ NMR spectra of biomass derived partial deoxygenation products. Raw FID files are also available for $75+$ compounds at the latest count. These data can be obtained at http://gagnegroup.web.unc.edu/sugars-spectroscopy/sugars.

\section{1,2-Deoxyglucose $(\mathbf{1})^{4}$}

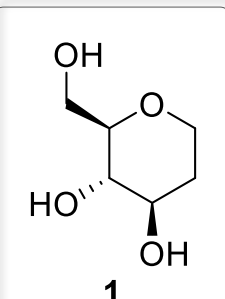

1
${ }^{1} \mathrm{H}$ NMR (600 MHz, $\left.\mathrm{CD}_{3} \mathrm{OD}\right) \delta 3.91(\mathrm{dd}, J=11.6,4.6 \mathrm{~Hz}, 1 \mathrm{H}), 3.84$ (dd, $J=$ $11.7,2.2 \mathrm{~Hz}, 1 \mathrm{H}), 3.63$ (dd, $J=11.7,5.7 \mathrm{~Hz}, 1 \mathrm{H}), 3.54-3.48(\mathrm{~m}, 1 \mathrm{H}), 3.48-$ $3.40(\mathrm{~m}, 1 \mathrm{H}), 3.18-3.09(\mathrm{~m}, 2 \mathrm{H}), 1.90(\mathrm{dd}, J=13.1,5.2 \mathrm{~Hz}, 1 \mathrm{H}), 1.59$ (dt, $J=$ $12.5,4.9 \mathrm{~Hz}, 1 \mathrm{H}) ;{ }^{13} \mathrm{C}\left\{{ }^{1} \mathrm{H}\right\}\left(151 \mathrm{MHz}, \mathrm{CD}_{3} \mathrm{OD}\right) \delta 82.4,74.1,73.6,66.6,63.2$, 35.1; HRMS(EI) Calculated for $\mathrm{C}_{6} \mathrm{H}_{12} \mathrm{O}_{4} \mathrm{Na}[\mathrm{M}+\mathrm{Na}]^{+}: 171.0628$, Found : 171.0634 . 
2-Deoxyglucitol (2)

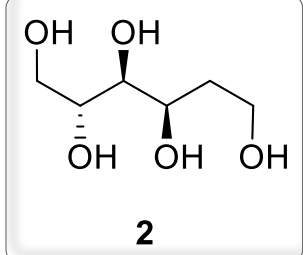

White solid (86\%, $11.5 \mathrm{mg}$, average of 2 runs); ${ }^{1} \mathrm{HNMR}\left(600 \mathrm{MHz}, \mathrm{CD}_{3} \mathrm{OD}\right) \delta$ $4.04-4.00(\mathrm{~m}, 1 \mathrm{H}), 3.79(\mathrm{dd}, J=11.2,3.6 \mathrm{~Hz}, 1 \mathrm{H}), 3.75-3.71(\mathrm{~m}, 2 \mathrm{H}), 3.70$ $-3.67(\mathrm{~m}, 1 \mathrm{H}), 3.62(\mathrm{dd}, J=11.2,6.0 \mathrm{~Hz}, 1 \mathrm{H}), 3.35(\mathrm{dd}, J=8.1,1.9 \mathrm{~Hz}, 1 \mathrm{H})$, $1.89-1.80(\mathrm{~m}, 1 \mathrm{H}), 1.72(\mathrm{ddd}, J=14.0,7.0,4.2 \mathrm{~Hz}, 1 \mathrm{H}) ;{ }^{13} \mathrm{C}\left\{{ }^{1} \mathrm{H}\right\} \mathrm{NMR}(151$ $\left.\mathrm{MHz}, \mathrm{CD}_{3} \mathrm{OD}\right) \delta 73.4,71.7,67.4,63.7,58.8,36.1$; HRMS(EI) calculated for $\mathrm{C}_{6} \mathrm{H}_{14} \mathrm{O}_{5} \mathrm{Na}[\mathrm{M}+\mathrm{Na}]^{+} ; 189.0734$, found: 189.0741 .

1,2-Deoxyglucitol $(3)^{4}$

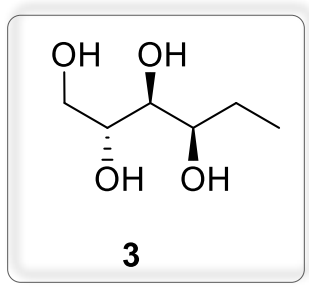

White solid (91\%, $221.0 \mathrm{mg}) ;{ }^{1} \mathrm{H}$ NMR $\left(600 \mathrm{MHz}, \mathrm{CD}_{3} \mathrm{OD}\right) \delta 3.79$ (dd, $J=$ $11.2,3.6 \mathrm{~Hz}, 1 \mathrm{H}), 3.73-3.65(\mathrm{~m}, 2 \mathrm{H}), 3.61(\mathrm{dd}, J=11.2,6.0 \mathrm{~Hz}, 1 \mathrm{H}), 3.38(\mathrm{~d}$, $J=7.9,1 \mathrm{H}), 1.67-1.46(\mathrm{~m}, 2 \mathrm{H}), 0.98(\mathrm{t}, J=7.5 \mathrm{~Hz}, 3 \mathrm{H}) ;{ }^{13} \mathrm{C}\left\{{ }^{1} \mathrm{H}\right\} \mathrm{NMR}(151$ $\left.\mathrm{MHz}, \mathrm{CD}_{3} \mathrm{OD}\right) \delta 72.7,71.8,71.6,63.7,26.1,9.4$; HRMS (EI) calculated for $\mathrm{C}_{6} \mathrm{H}_{14} \mathrm{O}_{4} \mathrm{Na}[\mathrm{M}+\mathrm{Na}]^{+}:$173.0785, found: 173.0791 .

\section{1,2-Deoxygalactose $(\mathbf{4})^{5}$}

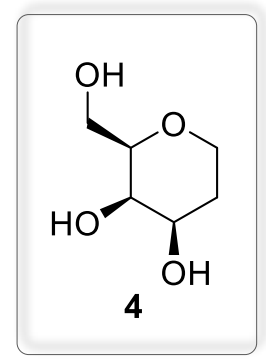

White solid (70\%, $8.3 \mathrm{mg}$, average of 3 runs); ${ }^{1} \mathrm{H}$ NMR (600 MHz, $\left.\mathrm{CD}_{3} \mathrm{OD}\right) \delta$ $3.96(\mathrm{ddd}, J=11.6,5.0,1.6 \mathrm{~Hz}, 1 \mathrm{H}), 3.75-3.69(\mathrm{~m}, 2 \mathrm{H}), 3.68-3.59(\mathrm{~m}, 2 \mathrm{H})$, 3.44 (ddd, $J=12.7,11.6,2.3 \mathrm{~Hz}, 1 \mathrm{H}), 3.33$ (ddd, $J=7.1,5.0,1.2 \mathrm{~Hz}, 1 \mathrm{H}), 1.92$ (qd, $J=12.5,5.1 \mathrm{~Hz}, 1 \mathrm{H}), 1.65-1.56(\mathrm{~m}, 1 \mathrm{H}) ;{ }^{13} \mathrm{C}\left\{{ }^{1} \mathrm{H}\right\} \mathrm{NMR}(151 \mathrm{MHz}$, $\mathrm{CD}_{3} \mathrm{OD}$ ) $\delta$ 80.9, 70.7, 69.8, 67.0, 63.4, 30.0; HRMS (EI) calculated for $\mathrm{C}_{6} \mathrm{H}_{12} \mathrm{O}_{4} \mathrm{Na}[\mathrm{M}+\mathrm{Na}]^{+}:$171.0628, found: 171.0634 .

\section{1,2-Deoxygalactitol (5)}

Clear film (98\%, $238.0 \mathrm{mg}) ;{ }^{1} \mathrm{H}$ NMR (600 MHz, $\left.\mathrm{CD}_{3} \mathrm{OD}\right){ }^{1} \mathrm{H}$ NMR $(600 \mathrm{MHz}$,<smiles>CCC(O)C(O)C(O)CO</smiles>
Methanol- $\left.d_{4}\right) \delta 3.90(\mathrm{td}, J=6.2,1.9 \mathrm{~Hz}, 1 \mathrm{H}), 3.64-3.59(\mathrm{~m}, 2 \mathrm{H}), 3.54(\mathrm{td}, J=$ 8.6, $3.0 \mathrm{~Hz}, 1 \mathrm{H}), 3.34(\mathrm{dd}, J=7.9,1.9 \mathrm{~Hz}, 1 \mathrm{H}), 1.85-1.74(\mathrm{~m}, 1 \mathrm{H}), 1.47-$ $1.35(\mathrm{~m}, 1 \mathrm{H}), 1.00$ (t, $J=7.4 \mathrm{~Hz}, 3 \mathrm{H}) ;{ }^{13} \mathrm{C}\left\{{ }^{1} \mathrm{H}\right\} \mathrm{NMR}\left(151 \mathrm{MHz}, \mathrm{CD}_{3} \mathrm{OD}\right) \delta$ 74.4, 74.1, 72.0, 64.9, 27.4, 10.2; HRMS (EI) calculated for $\mathrm{C}_{6} \mathrm{H}_{14} \mathrm{O}_{4} \mathrm{Na}$ $[\mathrm{M}+\mathrm{Na}]^{+}:$173.0785; found: $173.0792 ;[\alpha]_{\mathrm{D}}^{22}=+4.11(\mathrm{MeOH}, c=0.09)$. 
1-Deoxyglucose $(6)^{6}$

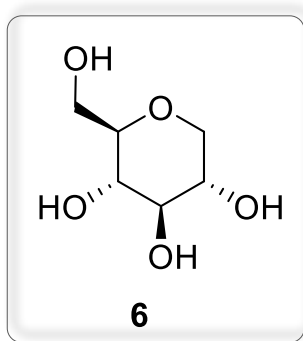

Clear film $\left(86 \%, 13.0 \mathrm{mg}\right.$, average of 2 runs); ${ }^{1} \mathrm{H}$ NMR (600 MHz, $\left.\mathrm{CD}_{3} \mathrm{OD}\right) \delta$ $3.89(\mathrm{dd}, J=11.1,5.4 \mathrm{~Hz}, 1 \mathrm{H}), 3.83(\mathrm{dd}, J=11.9,2.3 \mathrm{~Hz}, 1 \mathrm{H}), 3.61$ (dd, $J=$ $11.8,6.0 \mathrm{~Hz}, 1 \mathrm{H}), 3.45$ (ddd, $J=10.5,8.9,5.4 \mathrm{~Hz}, 1 \mathrm{H}), 3.29$ (t, $J=8.8 \mathrm{~Hz}, 1 \mathrm{H})$, $3.23(\mathrm{t}, J=9.2 \mathrm{~Hz}, 1 \mathrm{H}), 3.18-3.13(\mathrm{~m}, 2 \mathrm{H}) ;{ }^{13} \mathrm{C}\left\{{ }^{1} \mathrm{H}\right\}$ NMR $(151 \mathrm{MHz}$, $\left.\mathrm{CD}_{3} \mathrm{OD}\right) \delta 82.5,79.9,71.8,71.4,70.9,63.0$; HRMS (EI) calculated for $\mathrm{C}_{6} \mathrm{H}_{12} \mathrm{O}_{5} \mathrm{Na}[\mathrm{M}+\mathrm{Na}]^{+}:$187.0577; found: 187.0585 .

1-Deoxygalactose $(7)^{6}$

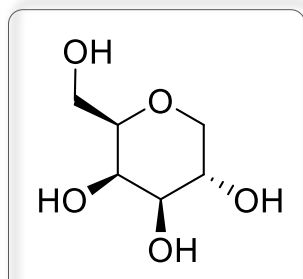

7

Clear film (93\%, $15.0 \mathrm{mg}) ;{ }^{1} \mathrm{H}$ NMR (600 MHz, CD 3 OD) $\delta 3.92$ (dd, $J=11.0$, $5.5 \mathrm{~Hz}, 1 \mathrm{H}), 3.86(\mathrm{dd}, J=3.4,1.1 \mathrm{~Hz}, 1 \mathrm{H}), 3.79$ (ddd, $J=10.4,9.4,5.5 \mathrm{~Hz}$, $1 \mathrm{H}), 3.71(\mathrm{dd}, J=11.4,7.1 \mathrm{~Hz}, 1 \mathrm{H}), 3.65(\mathrm{dd}, J=11.4,5.0 \mathrm{~Hz}, 1 \mathrm{H}), 3.42-3.36$ $(\mathrm{m}, 2 \mathrm{H}), 3.10(\mathrm{t}, J=10.7 \mathrm{~Hz}, 1 \mathrm{H}) ;{ }^{13} \mathrm{C}\left\{{ }^{1} \mathrm{H}\right\} \mathrm{NMR}\left(151 \mathrm{MHz}, \mathrm{CD}_{3} \mathrm{OD}\right) \delta 81.2$, 76.5, 71.2, 70.8, 68.4, 62.9; HRMS (EI) calculated for $\mathrm{C}_{6} \mathrm{H}_{12} \mathrm{O}_{5} \mathrm{Na}[\mathrm{M}+\mathrm{Na}]^{+}$: 187.0577; found: 187.0584 .

1-Deoxygalactitol $(\mathbf{8})^{7,8}$

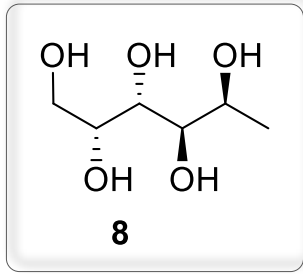

White solid (74\%, $189.0 \mathrm{mg}) ;{ }^{1} \mathrm{H}$ NMR (600 MHz, CD $\left.3 \mathrm{OD}\right) \delta 4.05$ (dq, $J=6.6$, $2.1 \mathrm{~Hz}, 1 \mathrm{H}), 3.91(\mathrm{dt}, J=6.3,1.7 \mathrm{~Hz}, 1 \mathrm{H}), 3.67-3.60(\mathrm{~m}, 3 \mathrm{H}), 3.43(\mathrm{dd}, J=$ 8.8, $2.1 \mathrm{~Hz}, 1 \mathrm{H}), 1.24(\mathrm{~d}, J=6.6 \mathrm{~Hz}, 3 \mathrm{H}) ;{ }^{13} \mathrm{C}\left\{{ }^{1} \mathrm{H}\right\} \mathrm{NMR}\left(151 \mathrm{MHz}, \mathrm{CD}_{3} \mathrm{OD}\right)$ $\delta$ 74.7, 72.1, 71.7, 67.6, 64.9, 19.9; HRMS (EI) calculated for $\mathrm{C}_{6} \mathrm{H}_{14} \mathrm{O}_{5} \mathrm{Na}$ $[\mathrm{M}+\mathrm{Na}]^{+}:$189.0734; found: 189.0740 .

\section{1-Deoxymannose $(9)^{6}$}

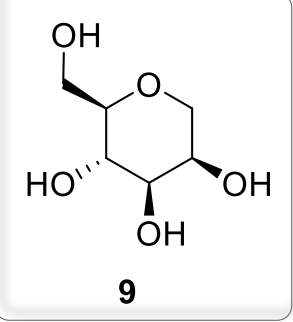

Clear film (79\%, $12.0 \mathrm{mg}) ;{ }^{1} \mathrm{H}$ NMR $\left(600 \mathrm{MHz}, \mathrm{CD}_{3} \mathrm{OD}\right) \delta 3.90$ (dd, $J=12.4$, $2.1 \mathrm{~Hz}, 1 \mathrm{H}), 3.87-3.82(\mathrm{~m}, 2 \mathrm{H}), 3.66(\mathrm{dd}, J=11.7,6.1 \mathrm{~Hz}, 1 \mathrm{H}), 3.59-3.53$ $(\mathrm{m}, 2 \mathrm{H}), 3.47$ (dd, $J=9.4,3.5 \mathrm{~Hz}, 1 \mathrm{H}), 3.14$ (ddd, $J=9.6,6.1,2.4 \mathrm{~Hz}, 1 \mathrm{H})$; ${ }^{13} \mathrm{C}\left\{{ }^{1} \mathrm{H}\right\} \mathrm{NMR}\left(151 \mathrm{MHz}, \mathrm{CD}_{3} \mathrm{OD}\right) \delta 82.6,75.8,71.3,71.0,69.0,63.1$; HRMS (EI) calculated for $\mathrm{C}_{6} \mathrm{H}_{12} \mathrm{O}_{5} \mathrm{Na}[\mathrm{M}+\mathrm{Na}]^{+}:$187.0577; found: 187.0585 . 


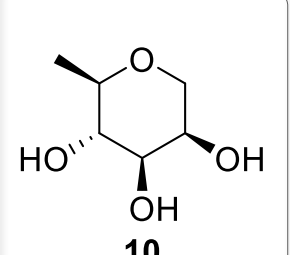

10

Clear film (97\%, $11.0 \mathrm{mg}$, average of 2 runs); ${ }^{1} \mathrm{H}$ NMR (600 MHz, $\left.\mathrm{CD}_{3} \mathrm{OD}\right) \delta$ $3.86-3.80(\mathrm{~m}, 2 \mathrm{H}), 3.55-3.50(\mathrm{~m}, 1 \mathrm{H}), 3.42(\mathrm{dd}, J=9.4,3.3 \mathrm{~Hz}, 1 \mathrm{H}), 3.33$ (t, $J=9.3 \mathrm{~Hz}, 1 \mathrm{H}), 3.15(\mathrm{dq}, J=9.1,6.1 \mathrm{~Hz}, 1 \mathrm{H}), 1.27(\mathrm{~d}, J=6.1 \mathrm{~Hz}, 3 \mathrm{H})$; ${ }^{13} \mathrm{C}\left\{{ }^{1} \mathrm{H}\right\}$ NMR (151 MHz, $\left.\mathrm{CD}_{3} \mathrm{OD}\right) \delta$ 78.1, 75.5, 74.3, 71.3, 71.0, 18.3; HRMS (EI) calculated for $\mathrm{C}_{6} \mathrm{H}_{12} \mathrm{O}_{4} \mathrm{Na}[\mathrm{M}+\mathrm{Na}]^{+}:$171.0628; found: 171.0634 .

\section{1,6-Deoxyglucose $(\mathbf{1 1})^{9}$}

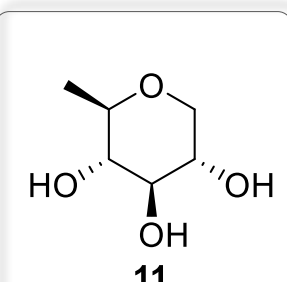

11

Clear film (55\%, $6.5 \mathrm{mg}$, average of 2 runs); $\left.{ }^{1} \mathrm{H} \mathrm{NMR} \mathrm{(600} \mathrm{MHz,} \mathrm{CD}_{3} \mathrm{OD}\right) \delta$ $3.82(\mathrm{dd}, J=11.1,5.4 \mathrm{~Hz}, 1 \mathrm{H}), 3.45(\mathrm{ddd}, J=10.6,9.0,5.4 \mathrm{~Hz}, 1 \mathrm{H}), 3.24(\mathrm{t}, J$ $=9.0 \mathrm{~Hz}, 1 \mathrm{H}), 3.21-3.16(\mathrm{~m}, 1 \mathrm{H}), 3.14(\mathrm{t}, J=10.9 \mathrm{~Hz}, 1 \mathrm{H}), 2.94(\mathrm{t}, J=9.1$ $\mathrm{Hz}, 1 \mathrm{H}), 1.23(\mathrm{~d}, J=6.2 \mathrm{~Hz}, 3 \mathrm{H}) ;{ }^{13} \mathrm{C}\left\{{ }^{1} \mathrm{H}\right\} \mathrm{NMR}\left(151 \mathrm{MHz}, \mathrm{CD}_{3} \mathrm{OD}\right) \delta 79.6$, 78.0, 77.2, 71.6, 70.9, 18.4; HRMS (EI) calculated for $\mathrm{C}_{6} \mathrm{H}_{12} \mathrm{O}_{4} \mathrm{Na}[\mathrm{M}+\mathrm{Na}]^{+}$: 171.0628; found: 171.0636 .

\section{6-Deoxyglucitol (12) $)^{10}$}

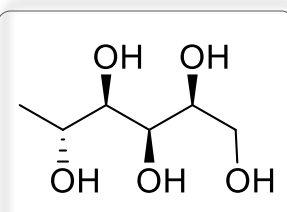

12

Clear film (47\%, $6.0 \mathrm{mg}$, average of 2 runs); ${ }^{1} \mathrm{H}$ NMR (600 MHz, $\left.\mathrm{CD}_{3} \mathrm{OD}\right) \delta$ $3.87-3.78(\mathrm{~m}, 2 \mathrm{H}), 3.76-3.71(\mathrm{~m}, 1 \mathrm{H}), 3.68(\mathrm{dd}, J=11.2,4.9 \mathrm{~Hz}, 1 \mathrm{H}), 3.59$ $(\mathrm{dd}, J=11.2,6.1 \mathrm{~Hz}, 1 \mathrm{H}), 3.40(\mathrm{dd}, J=7.3,2.4 \mathrm{~Hz}, 1 \mathrm{H}), 1.23(\mathrm{~d}, J=6.3 \mathrm{~Hz}$, 3H).; ${ }^{13} \mathrm{C}\left\{{ }^{1} \mathrm{H}\right\}$ NMR (151 MHz, $\left.\mathrm{CD}_{3} \mathrm{OD}\right) \delta 77.3,75.0,70.8,68.7,64.2,20.0$; HRMS (EI) calculated for $\mathrm{C}_{6} \mathrm{H}_{14} \mathrm{O}_{5} \mathrm{Na}[\mathrm{M}+\mathrm{Na}]^{+}:$189.0734; found: 189.0741 .

6-Deoxy-1,4-anhydro-L-galactitol (13)

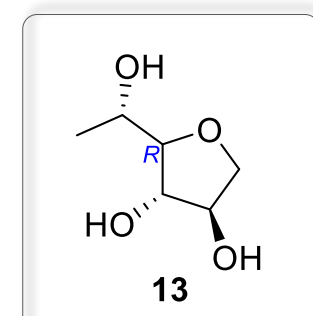

Clear film (85\%, $248.0 \mathrm{mg}) ;{ }^{1} \mathrm{H}$ NMR $\left(600 \mathrm{MHz}, \mathrm{CD}_{3} \mathrm{OD}\right) \delta 4.03$ (dt, $J=4.3$, $2.2 \mathrm{~Hz}, 1 \mathrm{H}), 3.97-3.90(\mathrm{~m}, 2 \mathrm{H}), 3.85(\mathrm{qd}, J=6.5,5.3 \mathrm{~Hz}, 1 \mathrm{H}), 3.78$ (dd, $J=$ 9.6, $2.2 \mathrm{~Hz}, 1 \mathrm{H}), 3.49$ (dd, $J=5.3,3.6 \mathrm{~Hz}, 1 \mathrm{H}), 1.22(\mathrm{~d}, J=6.5 \mathrm{~Hz}, 3 \mathrm{H})$; ${ }^{13} \mathrm{C}\left\{{ }^{1} \mathrm{H}\right\}$ NMR $\left(151 \mathrm{MHz}, \mathrm{CD}_{3} \mathrm{OD}\right) \delta 91.1,80.4,78.9,74.4,68.8,19.8$; HRMS (EI) calculated for $\mathrm{C}_{6} \mathrm{H}_{12} \mathrm{O}_{4} \mathrm{Na}[\mathrm{M}+\mathrm{Na}]^{+}$: 171.0628; found: 171.0634 ; $[\alpha]_{\mathrm{D}}^{23}=$ $+17.11(\mathrm{MeOH}, c=0.11)$. 


\section{1,4-Anhydroxylitol (14) $)^{11}$}

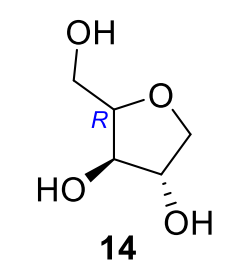

Clear film (84\%, $9.3 \mathrm{mg}$, average of 3 runs); ${ }^{1} \mathrm{H} \mathrm{NMR}\left(600 \mathrm{MHz}, \mathrm{CD}_{3} \mathrm{OD}\right) \delta$ $4.13(\mathrm{dt}, J=4.3,1.3 \mathrm{~Hz}, 1 \mathrm{H}), 4.12-4.09(\mathrm{~m}, 1 \mathrm{H}), 4.05(\mathrm{dd}, J=3.5,1.3 \mathrm{~Hz}$, 1H), 4.03 (ddd, $J=6.4,5.0,3.4 \mathrm{~Hz}, 1 \mathrm{H}), 3.79$ (dd, $J=11.5,5.0 \mathrm{~Hz}, 1 \mathrm{H}), 3.72$ $(\mathrm{dd}, J=11.5,6.3 \mathrm{~Hz}, 1 \mathrm{H}), 3.64(\mathrm{dd}, J=9.3,1.2 \mathrm{~Hz}, 1 \mathrm{H}) ;{ }^{13} \mathrm{C}\left\{{ }^{1} \mathrm{H}\right\}$ NMR $(151$ $\left.\mathrm{MHz}, \mathrm{CD}_{3} \mathrm{OD}\right) \delta 82.3,78.6,78.1,74.3,61.6$; HRMS (EI) calculated for $\mathrm{C}_{5} \mathrm{H}_{10} \mathrm{O}_{4} \mathrm{Na}[\mathrm{M}+\mathrm{Na}]^{+}:$157.0472; found: 157.0478 .

\section{Xylitol (15) ${ }^{4}$}

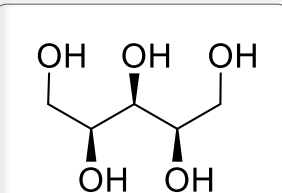

15

Clear film (88\%, $11.0 \mathrm{mg}$, average of 2 runs); ${ }^{1} \mathrm{H}$ NMR $\left(600 \mathrm{MHz}, \mathrm{CD}_{3} \mathrm{OD}\right) \delta$ $3.77-3.73(\mathrm{~m}, 2 \mathrm{H}), 3.69-3.59(\mathrm{~m}, 5 \mathrm{H}) ;{ }^{13} \mathrm{C}\left\{{ }^{1} \mathrm{H}\right\}\left(151 \mathrm{MHz}, \mathrm{CD}_{3} \mathrm{OD}\right) \delta 73.9$, 71.9, 64.3; HRMS (EI) calculated for $\mathrm{C}_{5} \mathrm{H}_{12} \mathrm{O}_{5} \mathrm{Na}[\mathrm{M}+\mathrm{Na}]^{+}: 175.0577$; found: 175.0584 .

\section{1-Deoxy-xylitol (16) $)^{12}$}

Clear film (97\%, $13.0 \mathrm{mg}$, average of 2 runs); ${ }^{1} \mathrm{H}$ NMR (600 MHz, $\left.\mathrm{CD}_{3} \mathrm{OD}\right) \delta$

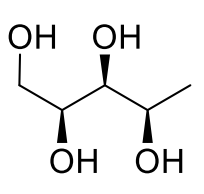

16 3.87 (qd, $J=6.4,5.1 \mathrm{~Hz}, 1 \mathrm{H}), 3.69$ (ddd, $J=6.2,5.3,3.1 \mathrm{~Hz}, 1 \mathrm{H}), 3.65(\mathrm{dd}, J$ $=11.0,5.3 \mathrm{~Hz}, 1 \mathrm{H}), 3.60(\mathrm{dd}, J=11.0,6.2 \mathrm{~Hz}, 1 \mathrm{H}), 3.35(\mathrm{dd}, J=5.2,3.1 \mathrm{~Hz}$, $1 \mathrm{H}), 1.20(\mathrm{~d}, J=6.4 \mathrm{~Hz}, 3 \mathrm{H}) ;{ }^{13} \mathrm{C}\left\{{ }^{1} \mathrm{H}\right\} \mathrm{NMR}\left(151 \mathrm{MHz}, \mathrm{CD}_{3} \mathrm{OD}\right) \delta 75.9,73.6$, 69.6, 64.5, 19.6; HRMS (EI) calculated for $\mathrm{C}_{5} \mathrm{H}_{12} \mathrm{O}_{4} \mathrm{Na}[\mathrm{M}+\mathrm{Na}]^{+}$: 159.0628; found: $159.0633 ;[\alpha]_{\mathrm{D}}^{24}=-3.48(\mathrm{MeOH}, c=0.11)$.

\section{1,6-Deoxygalactose $(\mathbf{1 7})^{6}$}

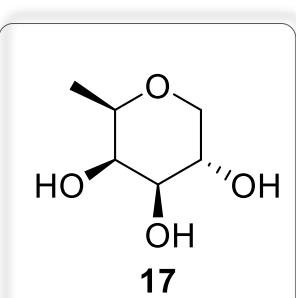

17

White solid (75\%, $11.5 \mathrm{mg}$, average of 2 runs); ${ }^{1} \mathrm{H}$ NMR (600 MHz, $\left.\mathrm{CD}_{3} \mathrm{OD}\right)$ $\delta 3.85$ (dd, $J=11.0,5.5 \mathrm{~Hz}, 1 \mathrm{H}), 3.74$ (ddd, $J=10.4,9.4,5.5 \mathrm{~Hz}, 1 \mathrm{H}), 3.63$ (dd, $J=3.4,1.1 \mathrm{~Hz}, 1 \mathrm{H}), 3.51(\mathrm{qd}, J=6.5,1.2 \mathrm{~Hz}, 1 \mathrm{H}), 3.38(\mathrm{dd}, J=9.4,3.4$ $\mathrm{Hz}, 1 \mathrm{H}), 3.08(\mathrm{t}, J=10.7 \mathrm{~Hz}, 1 \mathrm{H}), 1.22(\mathrm{~d}, J=6.5 \mathrm{~Hz}, 3 \mathrm{H}) ;{ }^{13} \mathrm{C}\left\{{ }^{1} \mathrm{H}\right\} \mathrm{NMR}(151$ $\left.\mathrm{MHz}, \mathrm{CD}_{3} \mathrm{OD}\right) \delta 76.7,76.5,73.4,71.2,68.1,17.1$; HRMS (EI) calculated for $\mathrm{C}_{6} \mathrm{H}_{12} \mathrm{O}_{4} \mathrm{Na}[\mathrm{M}+\mathrm{Na}]^{+}:$171.0628; found: 171.0634 . 
2,4-Bis-trimethylsilyl-1-deoxy-3,6-anhydrogalactose $(\mathbf{1 8})^{13}$

\begin{tabular}{|c|c|}
\hline & Clear film $(81 \%, 31.0 \mathrm{mg}) ;{ }^{1} \mathrm{H}$ NMR $\left(600 \mathrm{MHz}, \mathrm{CD}_{2} \mathrm{Cl}_{2}\right)$ \\
\hline & $\begin{array}{l}1 \mathrm{H}), 4.08(\mathrm{dd}, J=2.5 \mathrm{~Hz}, 1 \mathrm{H}), 4.03(\mathrm{~d}, J=10.0 \mathrm{~Hz}, 1 \mathrm{H}), 3.98(\mathrm{~d}, J=5.5 \mathrm{~Hz}, \\
1 \mathrm{H}), 3.94(\mathrm{dd}, J=10.0,3.1 \mathrm{~Hz}, 1 \mathrm{H}), 3.87(\mathrm{dd}, J=5.5,2.6 \mathrm{~Hz}, 1 \mathrm{H}), 3.77(\mathrm{dd}, J\end{array}$ \\
\hline $\mathrm{Me}_{3} \mathrm{SiO}$ & $=12.6,2.7 \mathrm{~Hz}, 1 \mathrm{H}), 0.15(\mathrm{~s}, 9 \mathrm{H}), 0.12(\mathrm{~s}, 9 \mathrm{H}) ;{ }^{13} \mathrm{C}\left\{{ }^{1} \mathrm{H}\right\} \mathrm{NMR}(151 \mathrm{MHz}$, \\
\hline & $\left.\mathrm{CD}_{2} \mathrm{Cl}_{2}\right) \delta 82.7,79.6,72.6,71.7,68.9,66.5,0.2,0.1 ; \mathrm{HRMS}(\mathrm{EI})$ calculated for \\
\hline & $\mathrm{Si}_{2} \mathrm{Na}[\mathrm{M}+\mathrm{Na}]^{+}: 313.1262$; found: 313.1273 \\
\hline
\end{tabular}

\section{References}

(1) Herrington, T. J.; Thom, A. J. W.; White, A. J. P.; Ashley, A. E. Novel $\mathrm{H}_{2}$ Activation by a Tris[3,5-Bis(Trifluoromethyl)Phenyl] Borane Frustrated Lewis Pair. Dalt. Trans. 2012, 41, 9019-9022.

(2) Bhat, A. S.; Gervay-Hague, J. Efficient Syntheses of $\beta$-Cyanosugars Using Glycosyl Iodides Derived from Per-O-Silylated Mono- and Disaccharides. Org. Lett. 2001, 3, 2081-2084.

(3) Seo, Y.; Gagné, M. R. Positional Selectivity in the Hydrosilylative Partial Deoxygenation of Disaccharides by Boron Catalysts. ACS Catal. 2018, 8, 81-85.

(4) Zhang, J.; Park, S.; Chang, S. Selective C-O Bond Cleavage of Sugars with Hydrosilanes Catalyzed by Piers' Borane Generated In Situ. Angew. Chemie - Int. Ed. 2017, 56, 1375713761 .

(5) Bhunia, A.; Schwardt, O.; Gäthje, H.; Gao, G. P.; Kelm, S.; Benie, A. J.; Hricovini, M.; Peters, T.; Ernst, B. Consistent Bioactive Conformation of the Neu5Aca(2-->3)Gal Epitope upon Lectin Binding. ChemBioChem 2008, 9, 2941-2945.

(6) Ohshima, Y.; Uchiyama, T.; Miyairi, S.; Ogawa, K.; Shishikura, K. An Efficient Method for the Preparation of 1,5-Anhydroalditol from Unprotected Carbohydrates via Glycopyranosyl Iodide. Tetrahedron Lett. 2016, 57, 5294-5296.

(7) Wiesler, W. T.; Nakanishi, K. Relative and Absolute Configurational Assignments of Acyclic Polyols by Circular Dichroism. 1. Rationale for a Simple Procedure Based on the Exciton Chirality Method. J. Am. Chem. Soc. 1989, 111, 9205-9213.

(8) Yoshihara, A.; Haraguchi, S.; Gullapalli, P.; Rao, D.; Morimoto, K.; Takata, G.; Jones, N.; Jenkinson, S. F.; Wormald, M. R.; Dwek, R. A.; et al. Isomerization of Deoxyhexoses: Green Bioproduction of 1-Deoxy-D-Tagatose from 1-Fucose and of 6-Deoxy-D-Tagatose from D-Fucose Using Enterobacter Agglomerans Strain 221e. Tetrahedron Asymmetry 2008, 19, 739-745.

(9) Murali, R.; Nagarajan, M. Synthesis of Partially Protected 1,5-Anhydroalditols by Hydroboration of Glycals. Carbohydr. Res. 1996, 280, 351-355. 
(10) Liu, Z.; Yoshihara, A.; Jenkinson, S. F.; Wormald, M. R.; Estévez, R. J.; Fleet, G. W. J.; Izumori, K. Triacetonide of Glucoheptonic Acid in the Scalable Syntheses of D-Gulose, 6Deoxy-D-Gulose, 1-Glucose, 6-Deoxy-1-Glucose, and Related Sugars. Org. Lett. 2016, 18, 4112-4115.

(11) Barbaro, P.; Liguori, F.; Moreno-Marrodan, C. Selective Direct Conversion of $\mathrm{C}_{5}$ and $\mathrm{C}_{6}$ Sugars to High Added-Value Chemicals by a Bifunctional, Single Catalytic Body. Green Chem. 2016, 18, 2935-2940.

(12) Takai, K.; Heathcock, C. H. Acyclic Stereoselection. 32. Synthesis and Characterization of the Diastereomeric (4S)-Pentane-1,2,3,4-Tetrols. J. Org. Chem. 1985, 50, 3247-3251.

(13) Houseknecht, J. B.; Lowary, T. L. Oligofuranosides Containing Conformationally Restricted Residues: Synthesis and Conformational Analysis. J. Org. Chem. 2002, 67, 4150-4164. 

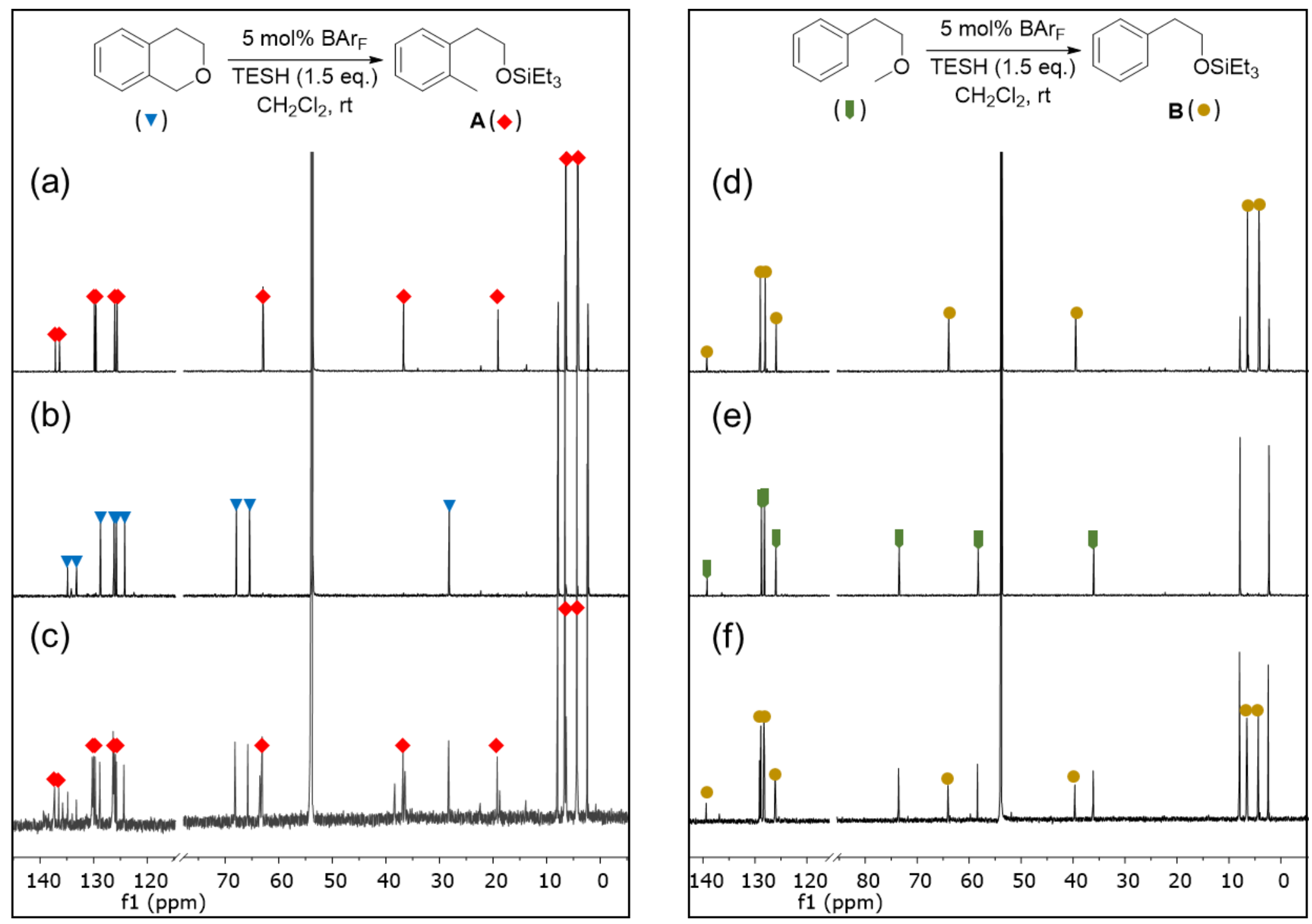

Figure S1. ${ }^{13} \mathrm{C}$ NMR spectra during the reaction of isochroman (Left) (a) with $\mathrm{B}\left(\mathrm{C}_{6} \mathrm{~F}_{5}\right)_{3}$ after $1 \mathrm{~h}$; (b) with $\mathrm{BAr}_{3,5-\mathrm{CF} 3}, 1 \mathrm{~h}$ later and (c) $48 \mathrm{~h}$ later; (Right) methoxyethylbenzene (d) with $\mathrm{B}\left(\mathrm{C}_{6} \mathrm{~F}_{5}\right)_{3}$ after $1 \mathrm{~h}$; (e) with $\mathrm{BAr}_{3,5-\mathrm{CF} 3}, 1 \mathrm{~h}$ later and (f) $48 \mathrm{~h}$ later. 

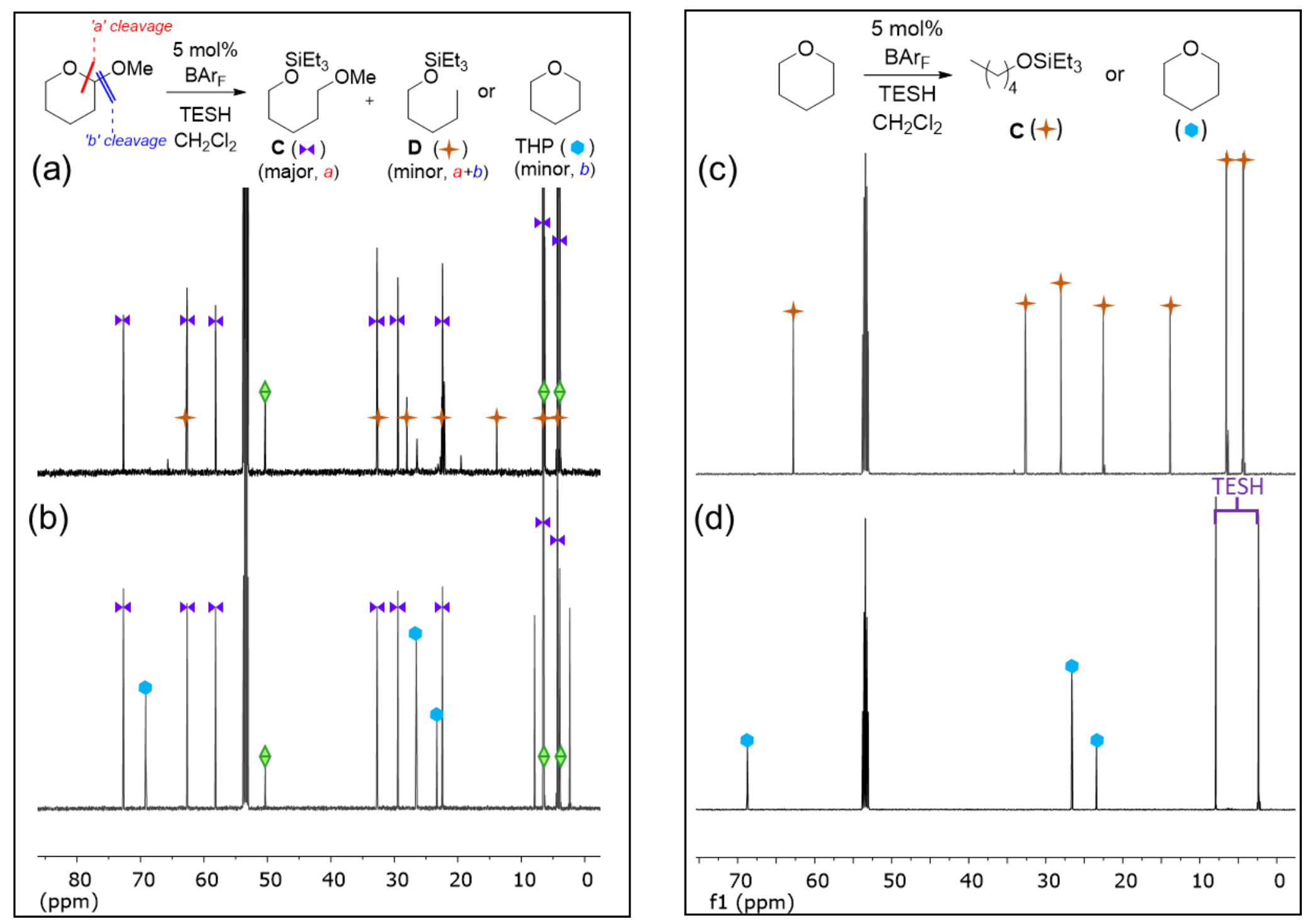

Figure S2. ${ }^{13} \mathrm{C}$ NMR spectra during the reaction of (Left) 2-methoxytetrahydropyran (a) with $\mathrm{B}\left(\mathrm{C}_{6} \mathrm{~F}_{5}\right)_{3}, 5$ h; (b) with $\mathrm{BAr} \mathrm{r}_{3,5-\mathrm{CF} 3} 5 \mathrm{~h}$;

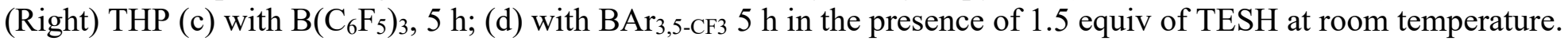




$$
\mathrm{Si}=\mathrm{SiMe}_{3} \quad \mathrm{Si}=\mathrm{SiEt}_{3}
$$

(a) $\mathrm{R}_{3} \mathrm{Si}$-1-deoxyglucose (Si-6)

(b) $\mathrm{R}_{3} \mathrm{Si}$-1-deoxygalactose (Si-7)
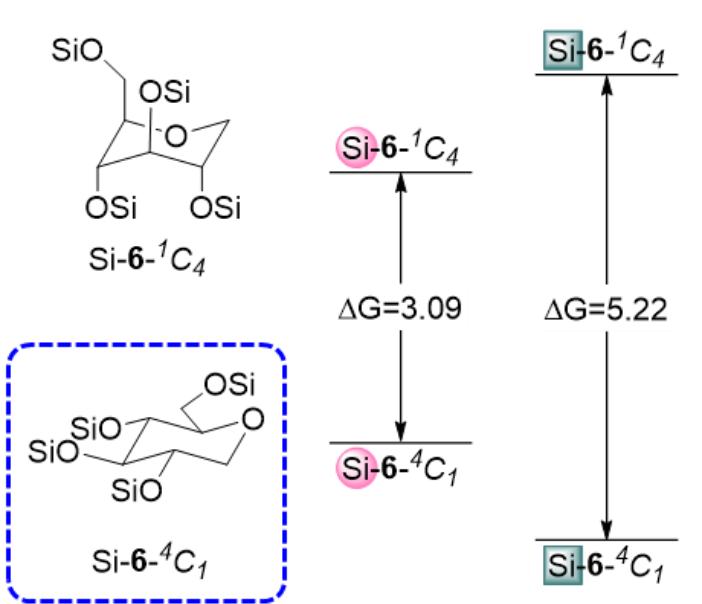

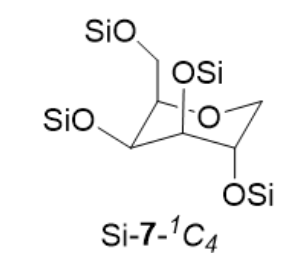

$$
\frac{S_{i-7-}-{ }^{1} C_{4}}{\Delta G=0.46}
$$
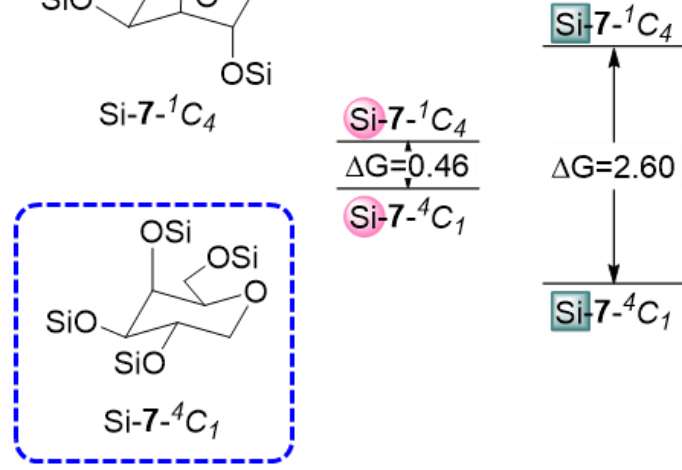

(c) $\mathrm{R}_{3} \mathrm{Si}$-1-deoxymannose (Si-8)

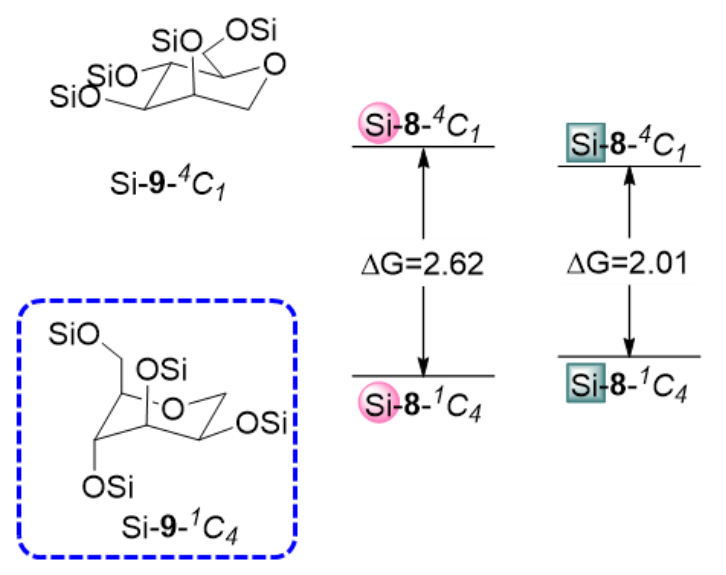

Figure S3. Free energy comparisons (kcal/mol) of two chair conformations $\left({ }^{1} C_{4}\right.$ and $\left.{ }^{4} C_{1}\right)$ of persilylated 1-deoxysugars; (a) $\mathrm{R}_{3} \mathrm{Si}$-1deoxyglucose (Si-6), (b) $\mathrm{R}_{3} \mathrm{Si}$-1-deoxygalactose (Si-7) and (c) $\mathrm{R}_{3} \mathrm{Si}$-1-deoxymannose (Si-8). The free energies were calculated by DFT methods (see computational details on $\mathrm{S} 46$ ); (M06-2X/6-31+G(d,p), $\mathrm{CPCM} \mathrm{CH}_{2} \mathrm{Cl}_{2}$. 


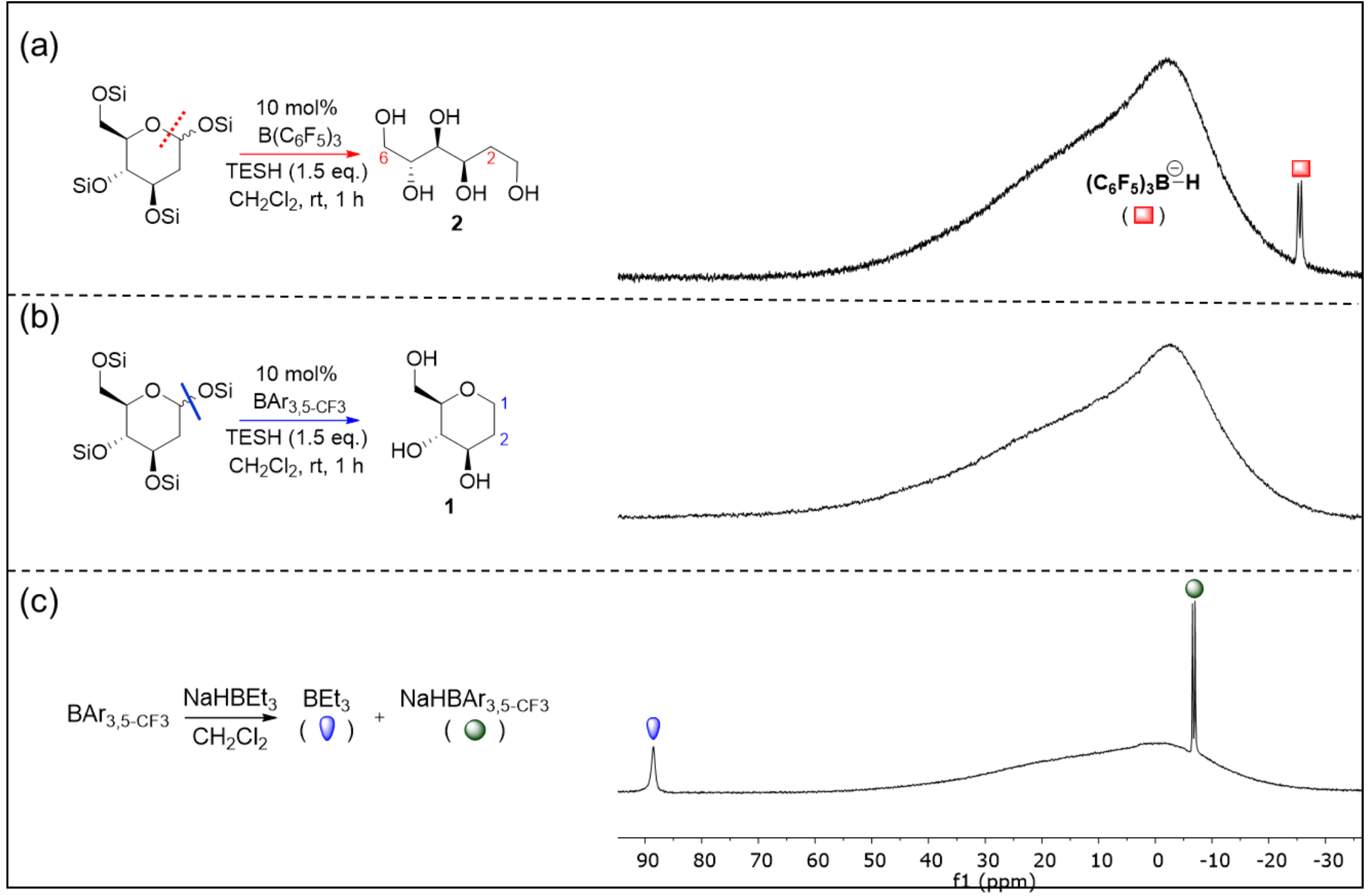

Figure S4. In situ ${ }^{11} \mathrm{~B}$ NMR spectra of each reaction shown in Figure (a) (c). 
${ }^{1} \mathrm{H}$ NMR $\left(600 \mathrm{MHz}, \mathrm{CD}_{3} \mathrm{OD}\right)$

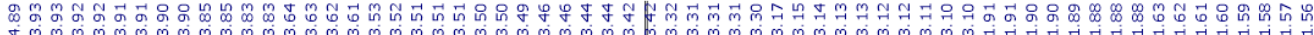
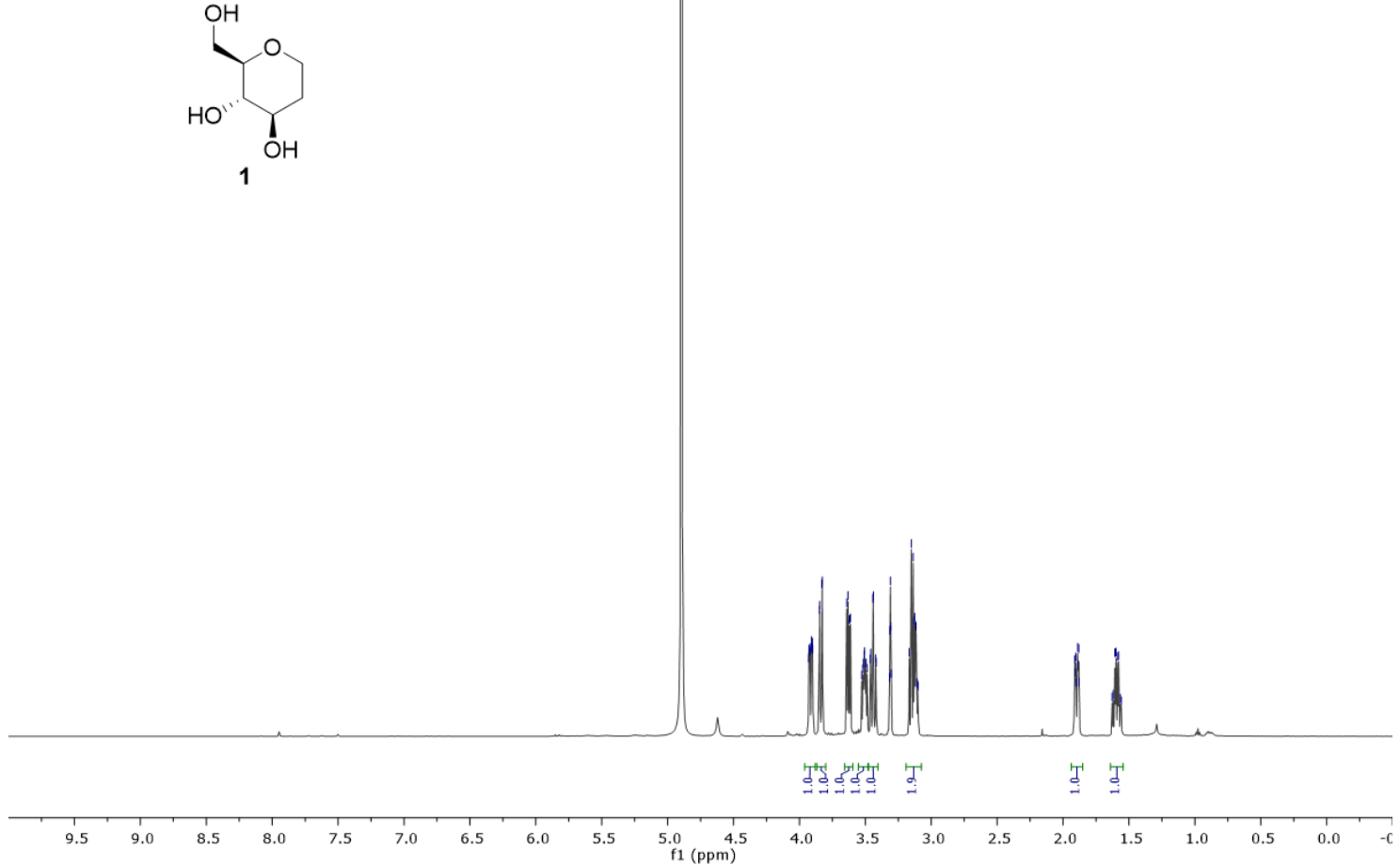

${ }^{13} \mathrm{C}$ NMR $\left(151 \mathrm{MHz}, \mathrm{CD}_{3} \mathrm{OD}\right)$

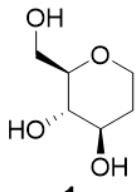

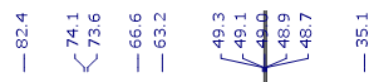

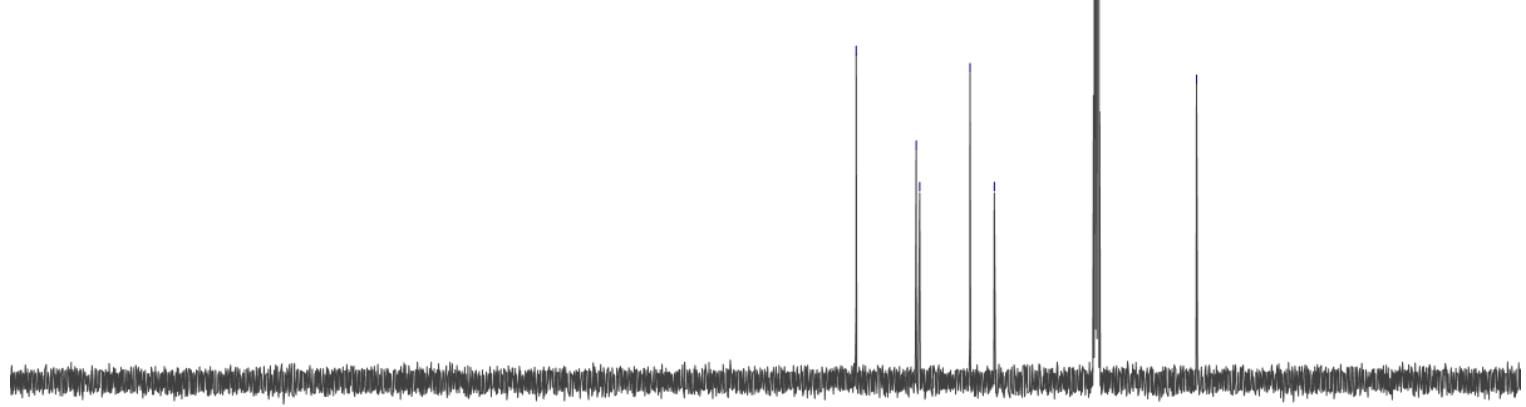

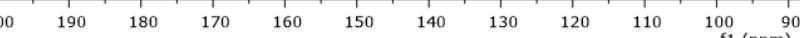


${ }^{1} \mathrm{H}$ NMR $\left(600 \mathrm{MHz}, \mathrm{CD}_{3} \mathrm{OD}\right)$

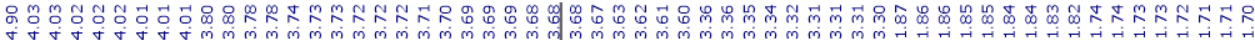<smiles>OCCC(O)C(O)CO</smiles>
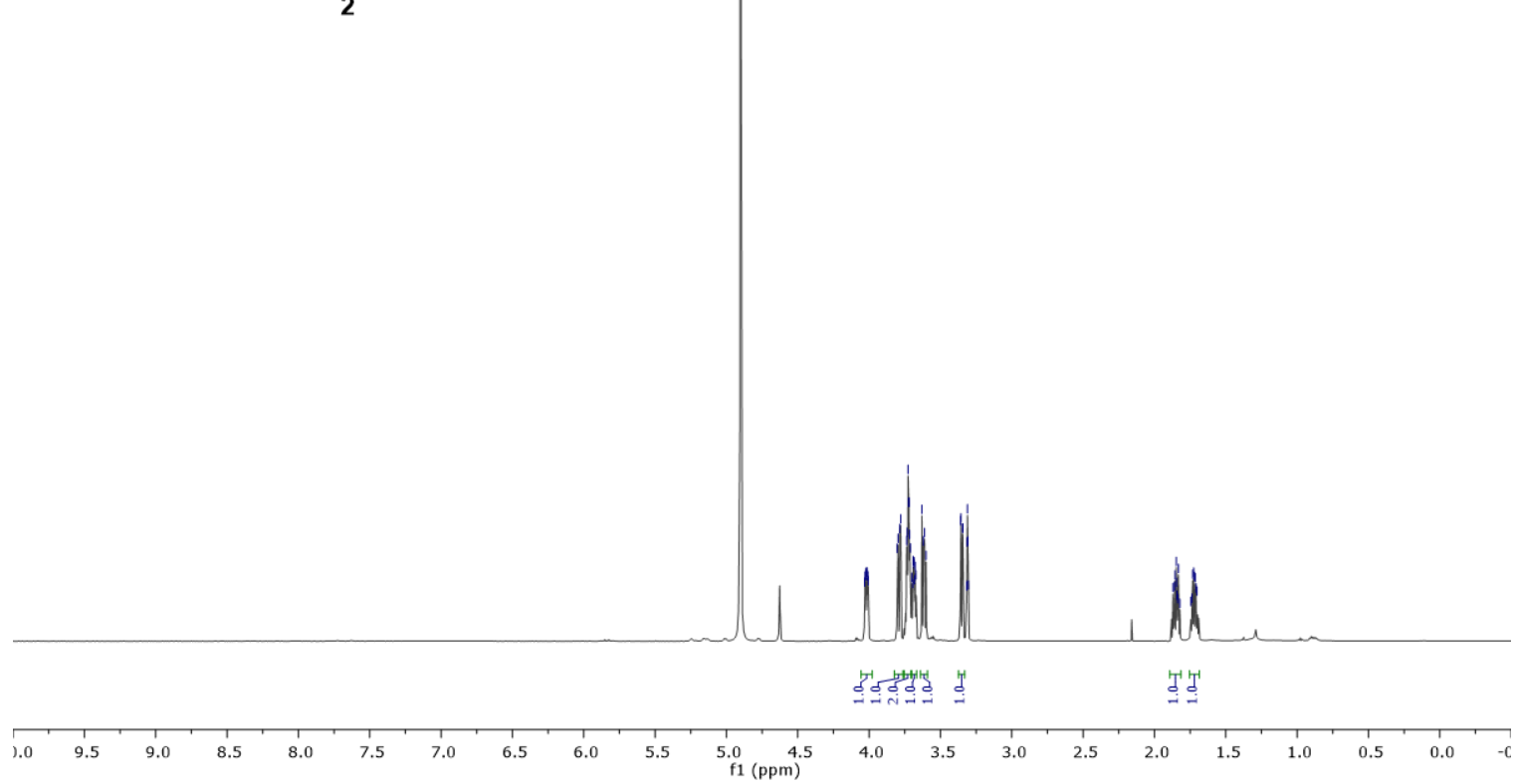

${ }^{13} \mathrm{C}$ NMR $\left(151 \mathrm{MHz}, \mathrm{CD}_{3} \mathrm{OD}\right)$<smiles>OCCC(O)C(O)CO</smiles>
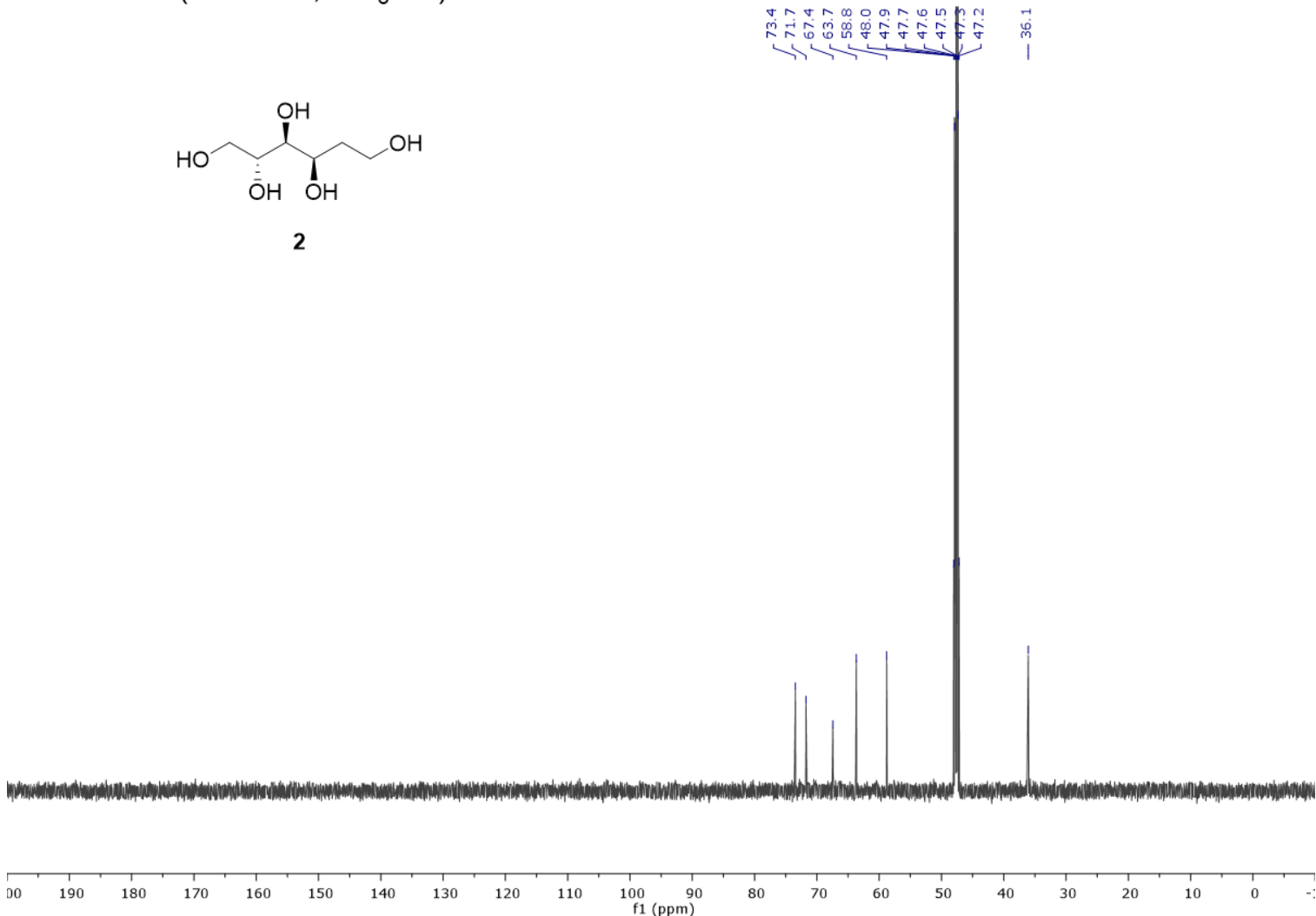
${ }^{1} \mathrm{H}$ NMR $\left(600 \mathrm{MHz}, \mathrm{CD}_{3} \mathrm{OD}\right)$

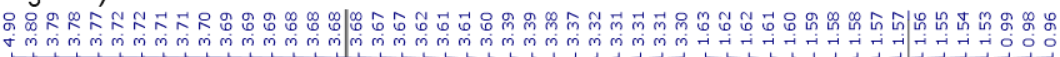

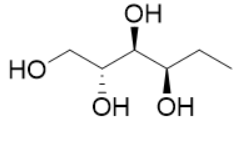

3

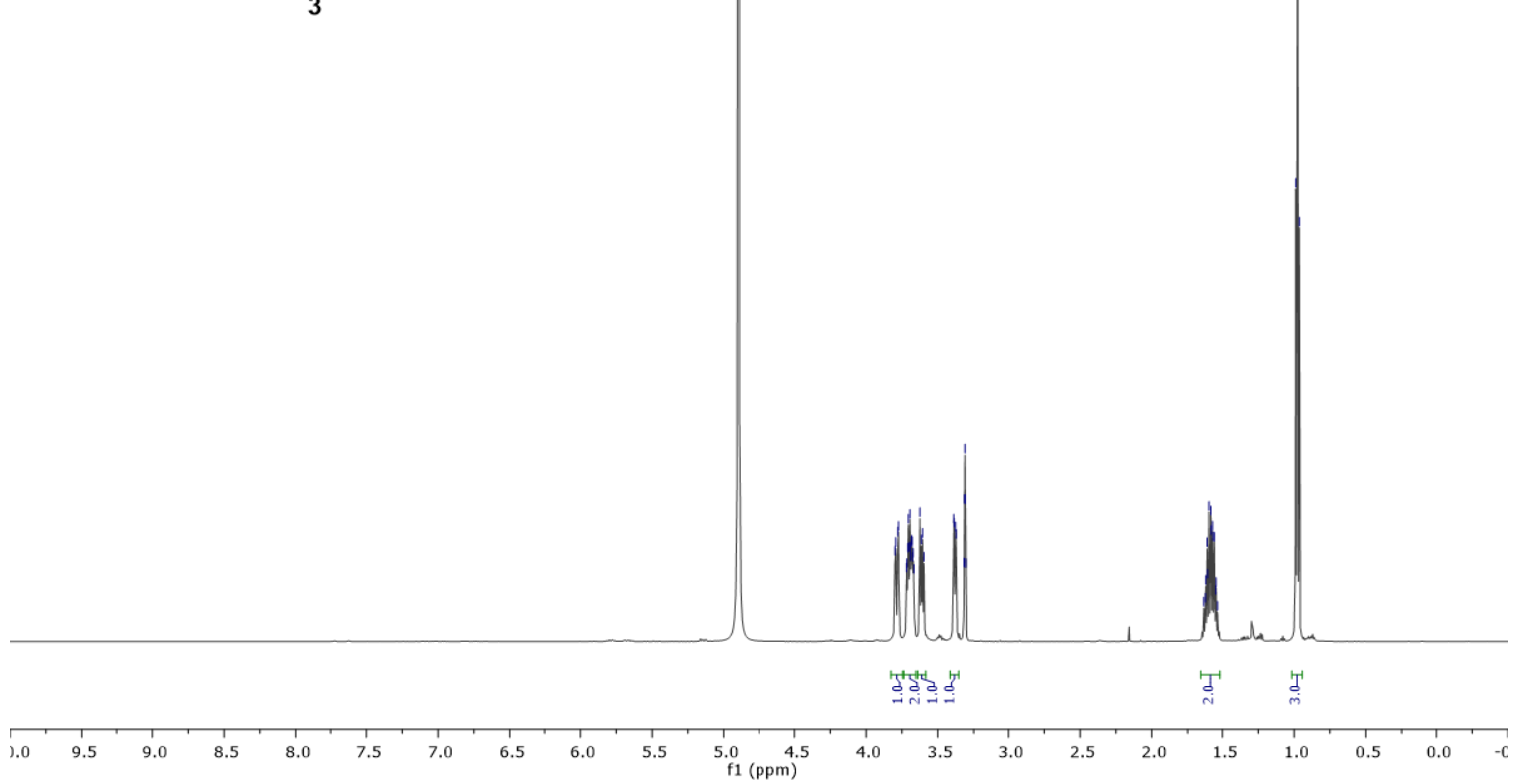

${ }^{13} \mathrm{C}$ NMR $\left(151 \mathrm{MHz}, \mathrm{CD}_{3} \mathrm{OD}\right)$

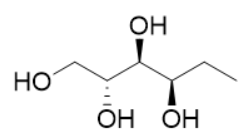

3 
${ }^{1} \mathrm{H}$ NMR $\left(600 \mathrm{MHz}, \mathrm{CD}_{3} \mathrm{OD}\right)$

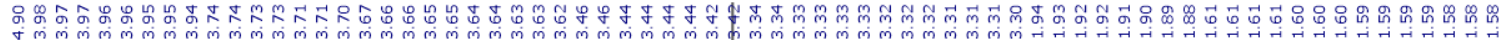
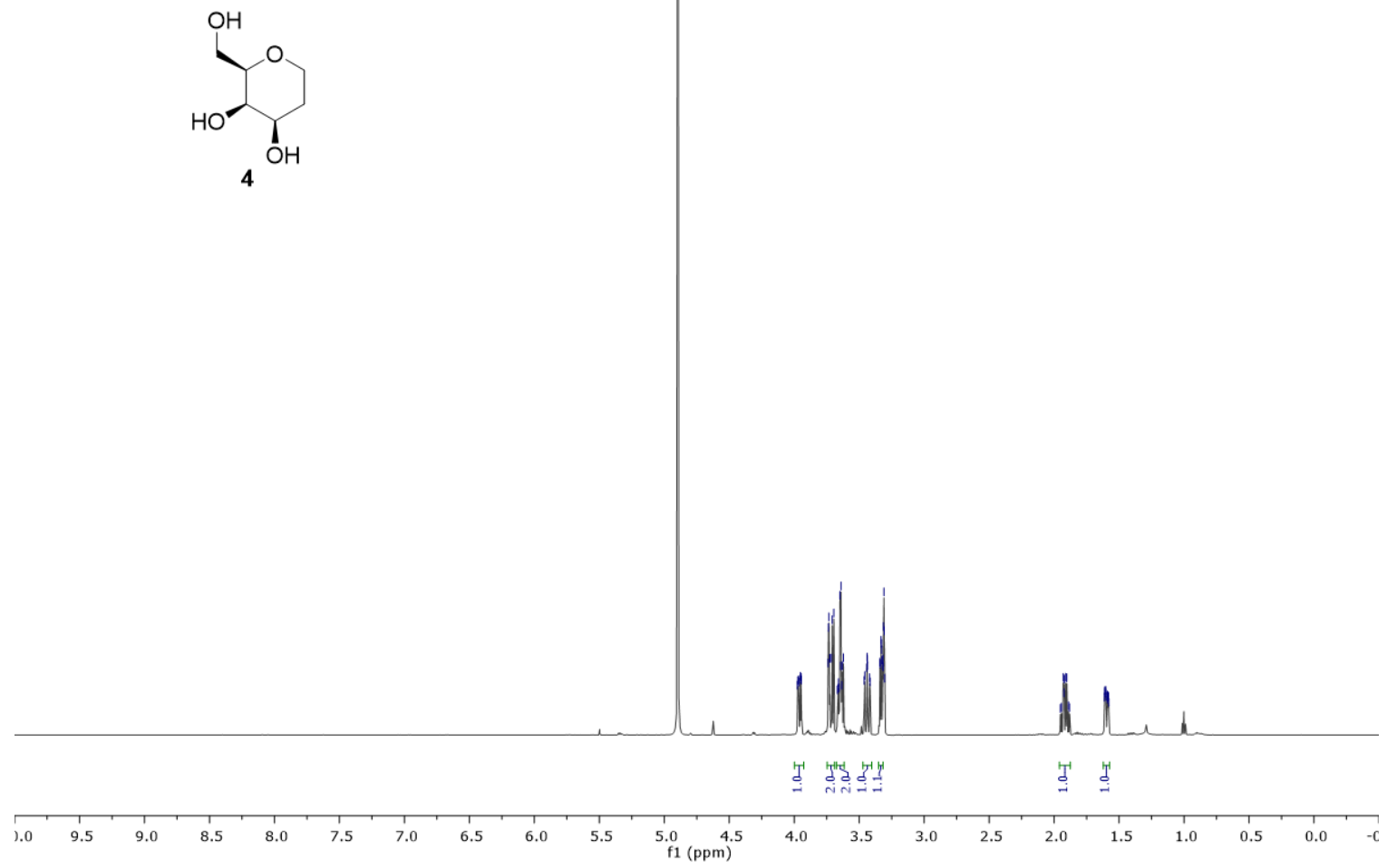

${ }^{13} \mathrm{C}$ NMR $\left(151 \mathrm{MHz}, \mathrm{CD}_{3} \mathrm{OD}\right)$
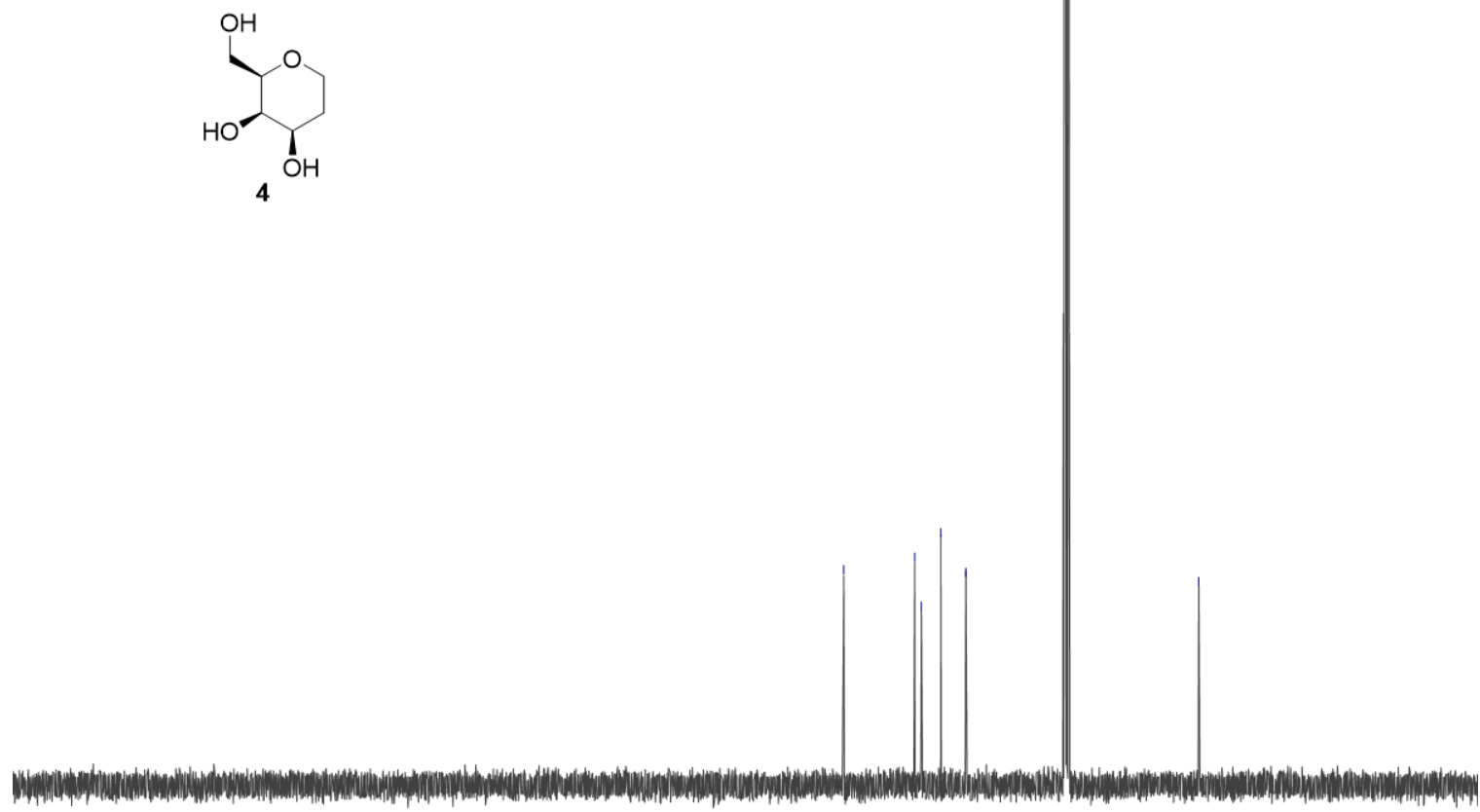
${ }^{1} \mathrm{H}$ NMR $\left(600 \mathrm{MHz}, \mathrm{CD}_{3} \mathrm{OD}\right)$

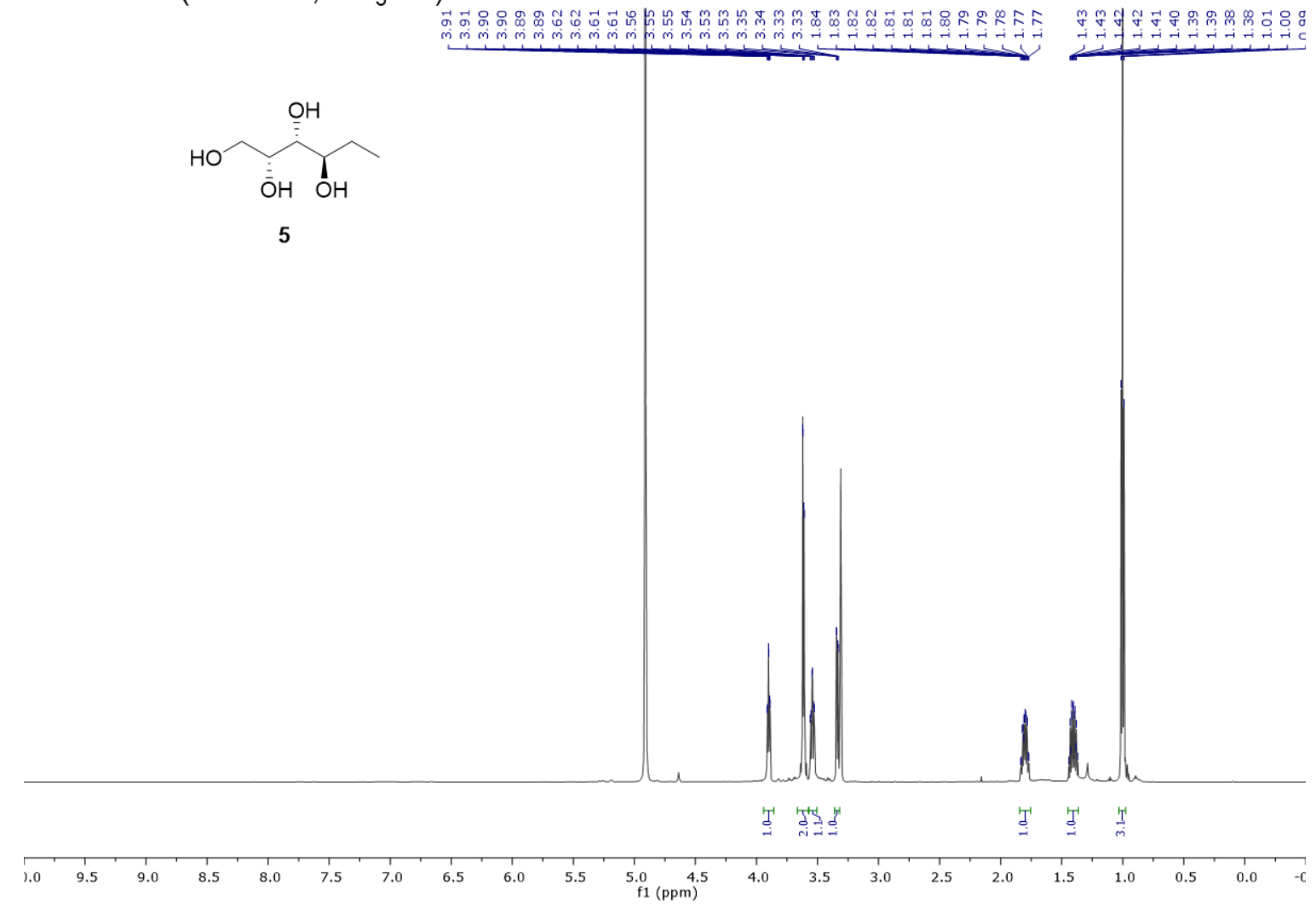

${ }^{13} \mathrm{C}$ NMR $\left(151 \mathrm{MHz}, \mathrm{CD}_{3} \mathrm{OD}\right)$

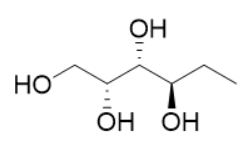

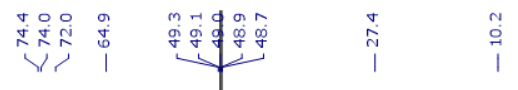

5 
2D NMR (COSY, $\left.\mathrm{CD}_{3} \mathrm{OD}\right)$

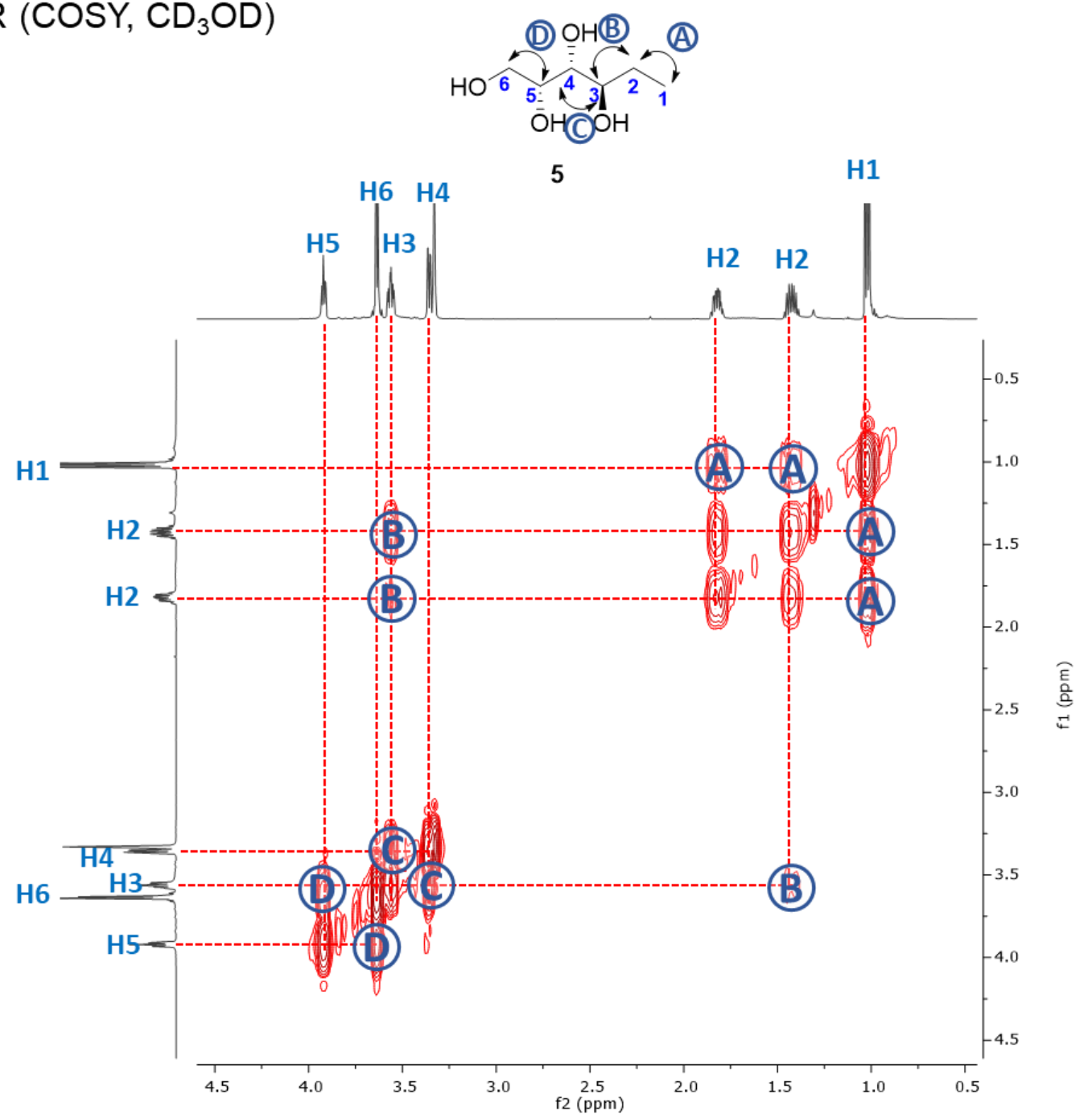




\section{D NMR (HSQC, $\left.\mathrm{CD}_{3} \mathrm{OD}\right)$}

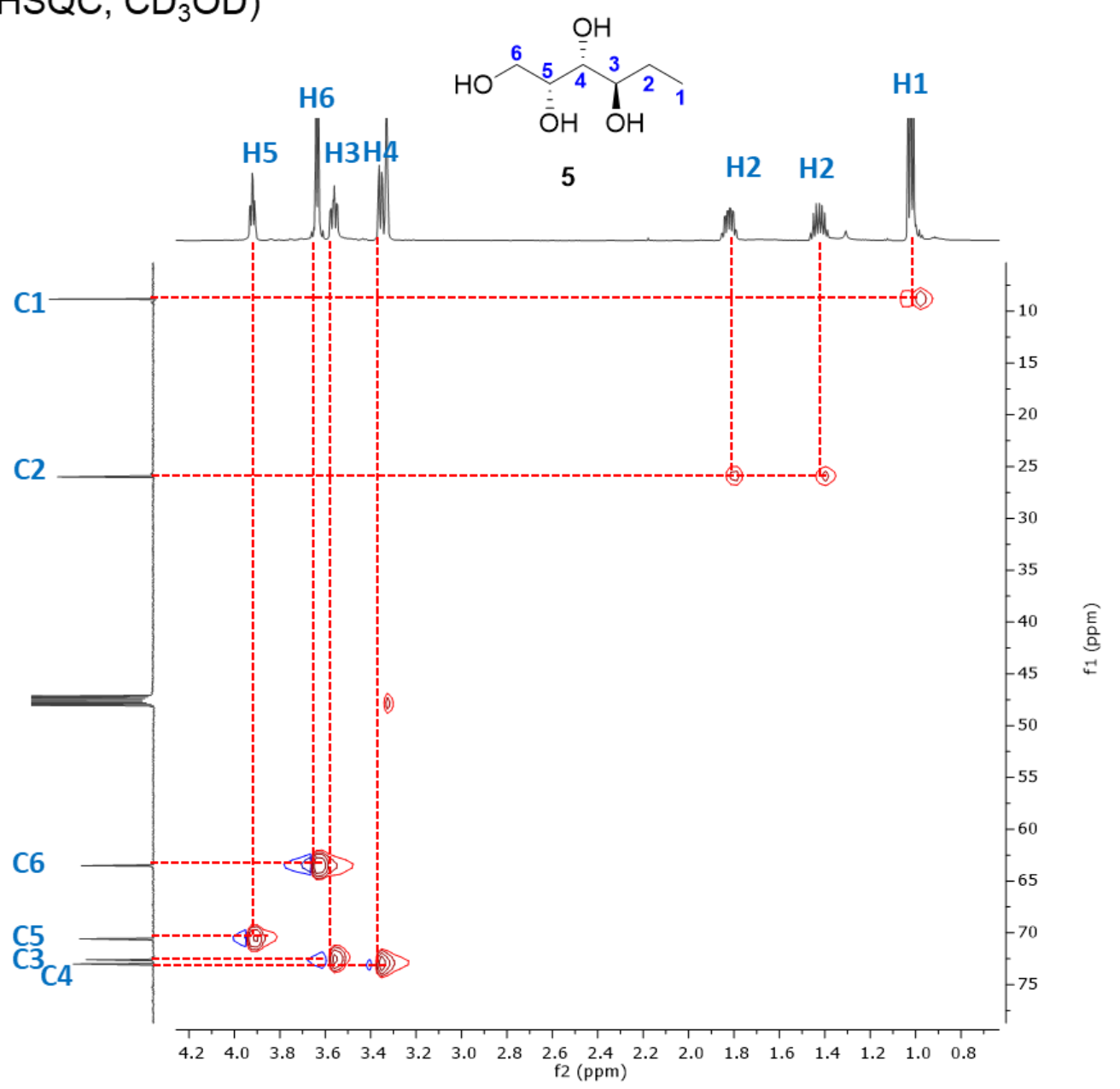


2D NMR (HMBC, $\left.\mathrm{CD}_{3} \mathrm{OD}\right)$

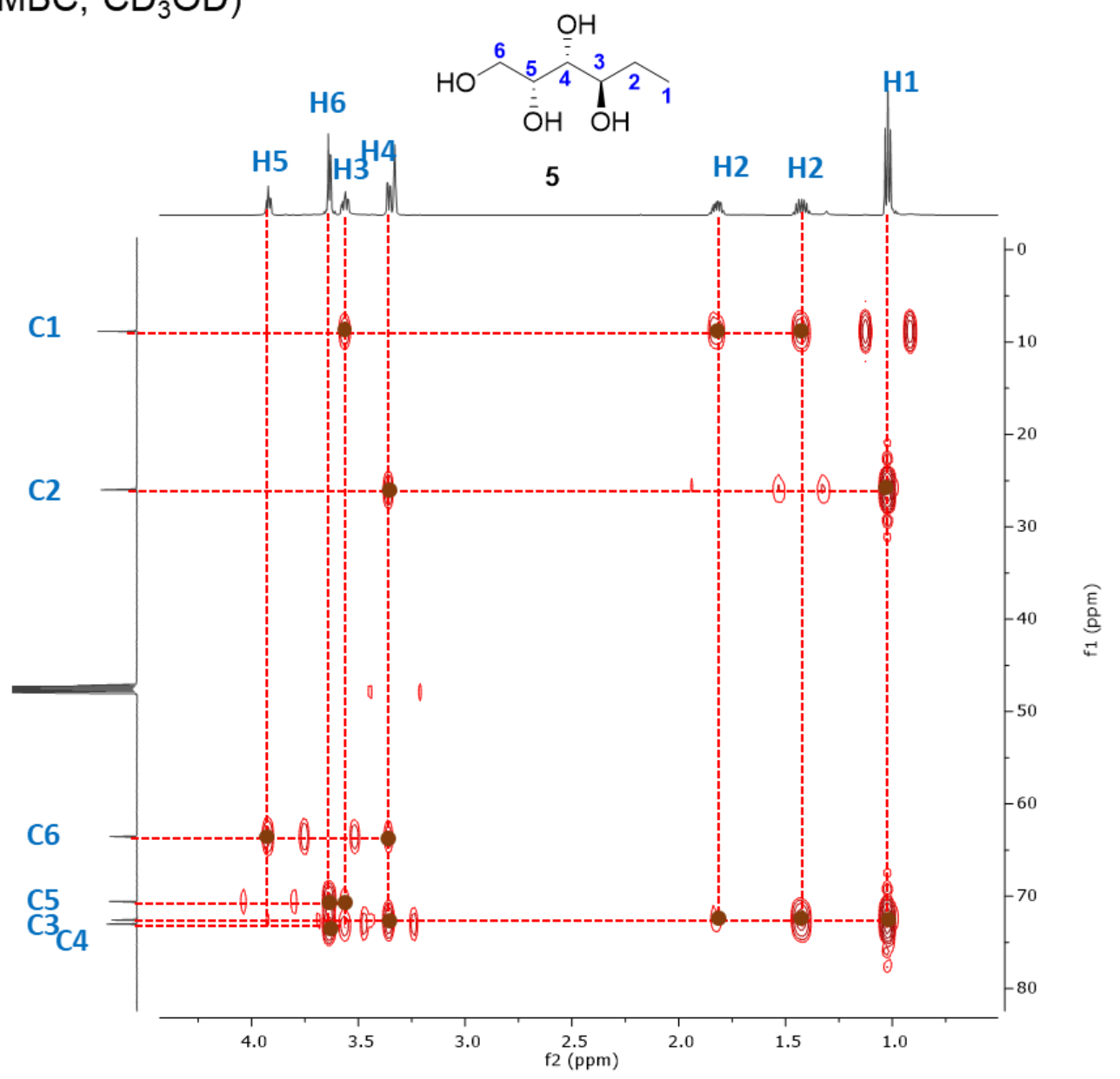


${ }^{1} \mathrm{H}$ NMR $\left(600 \mathrm{MHz}, \mathrm{CD}_{3} \mathrm{OD}\right)$

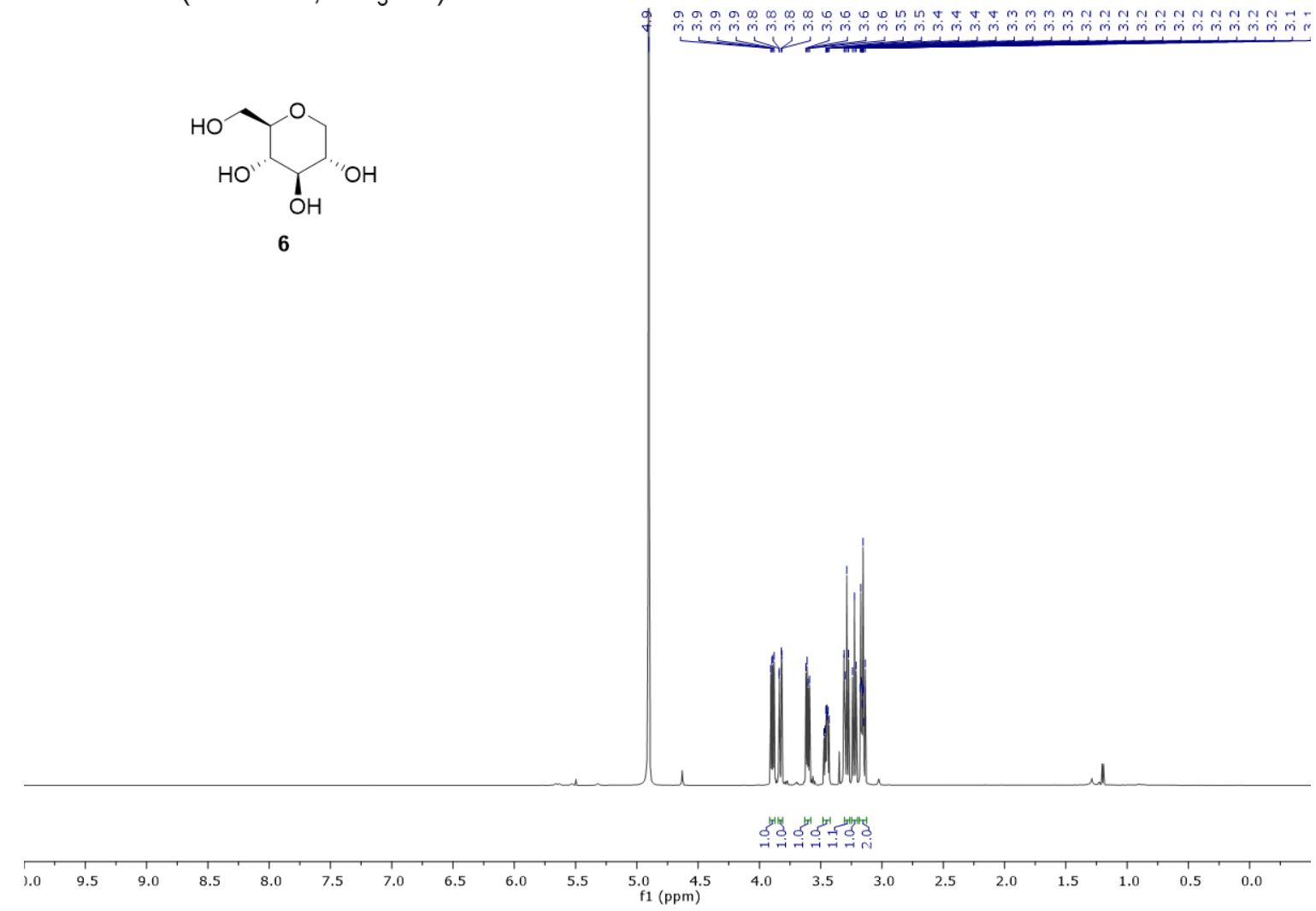

${ }^{13} \mathrm{C}$ NMR $\left(151 \mathrm{MHz}, \mathrm{CD}_{3} \mathrm{OD}\right)$
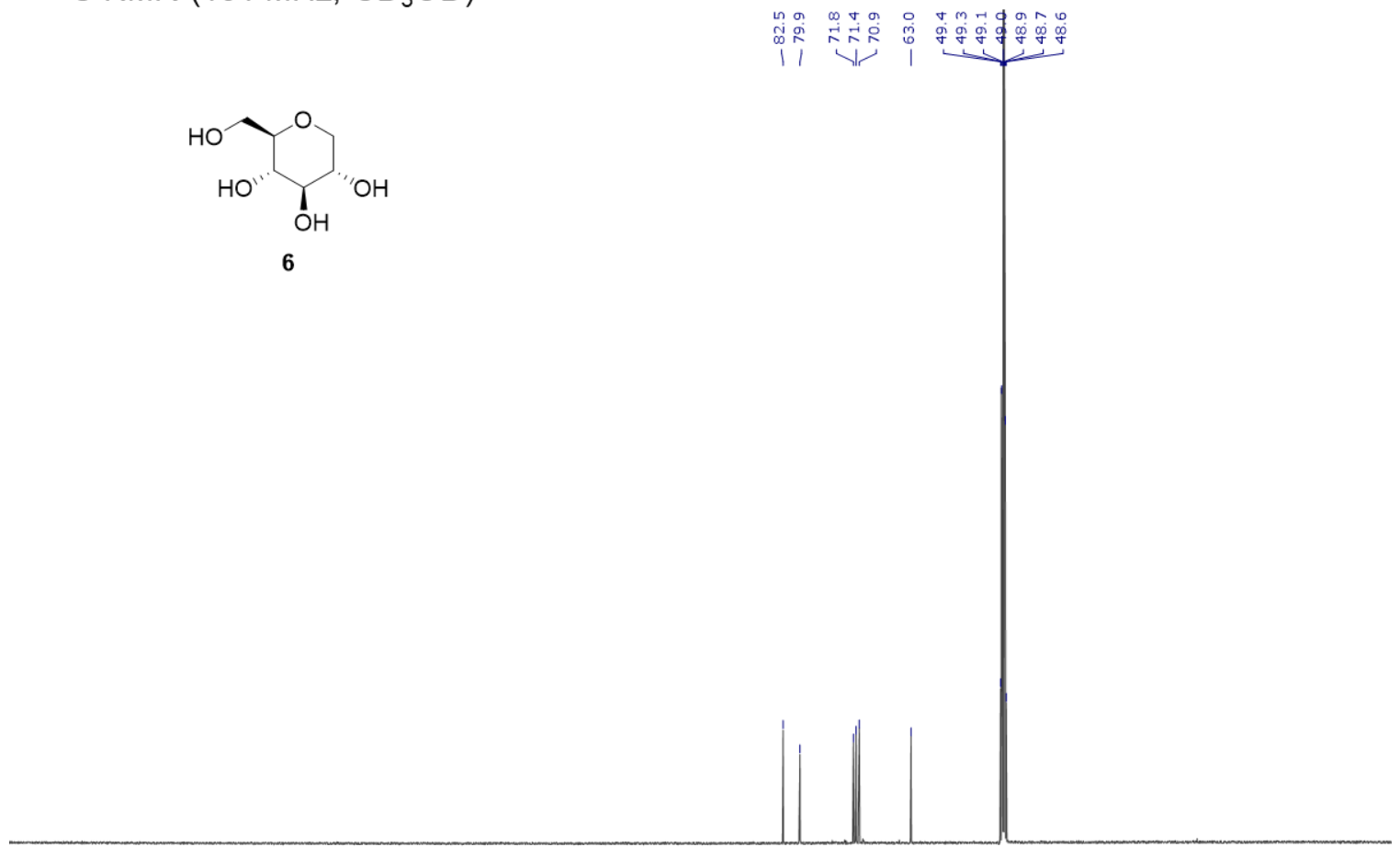
${ }^{1} \mathrm{H}$ NMR $\left(600 \mathrm{MHz}, \mathrm{CD}_{3} \mathrm{OD}\right)$

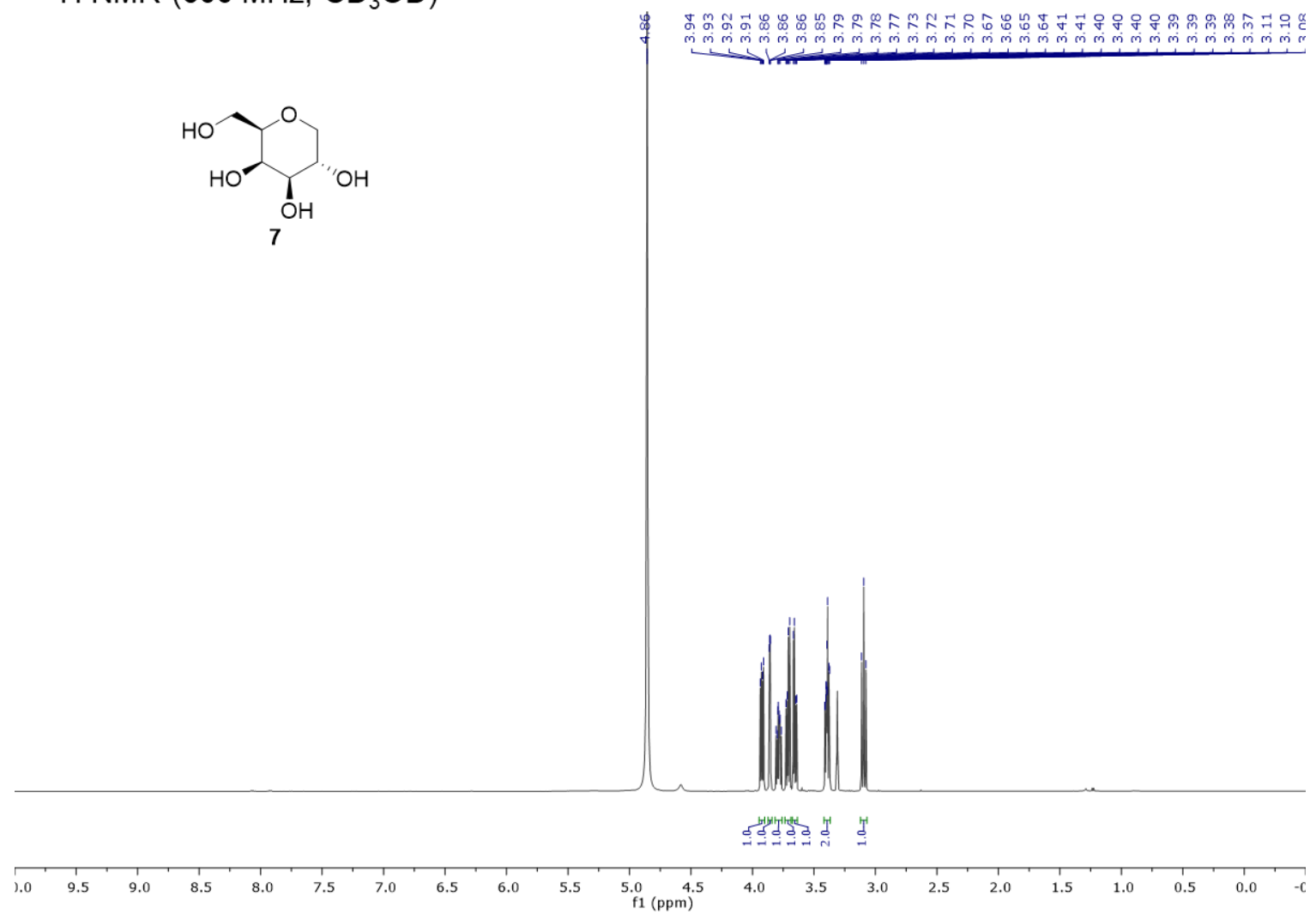

${ }^{13} \mathrm{C}$ NMR $\left(151 \mathrm{MHz}, \mathrm{CD}_{3} \mathrm{OD}\right)$
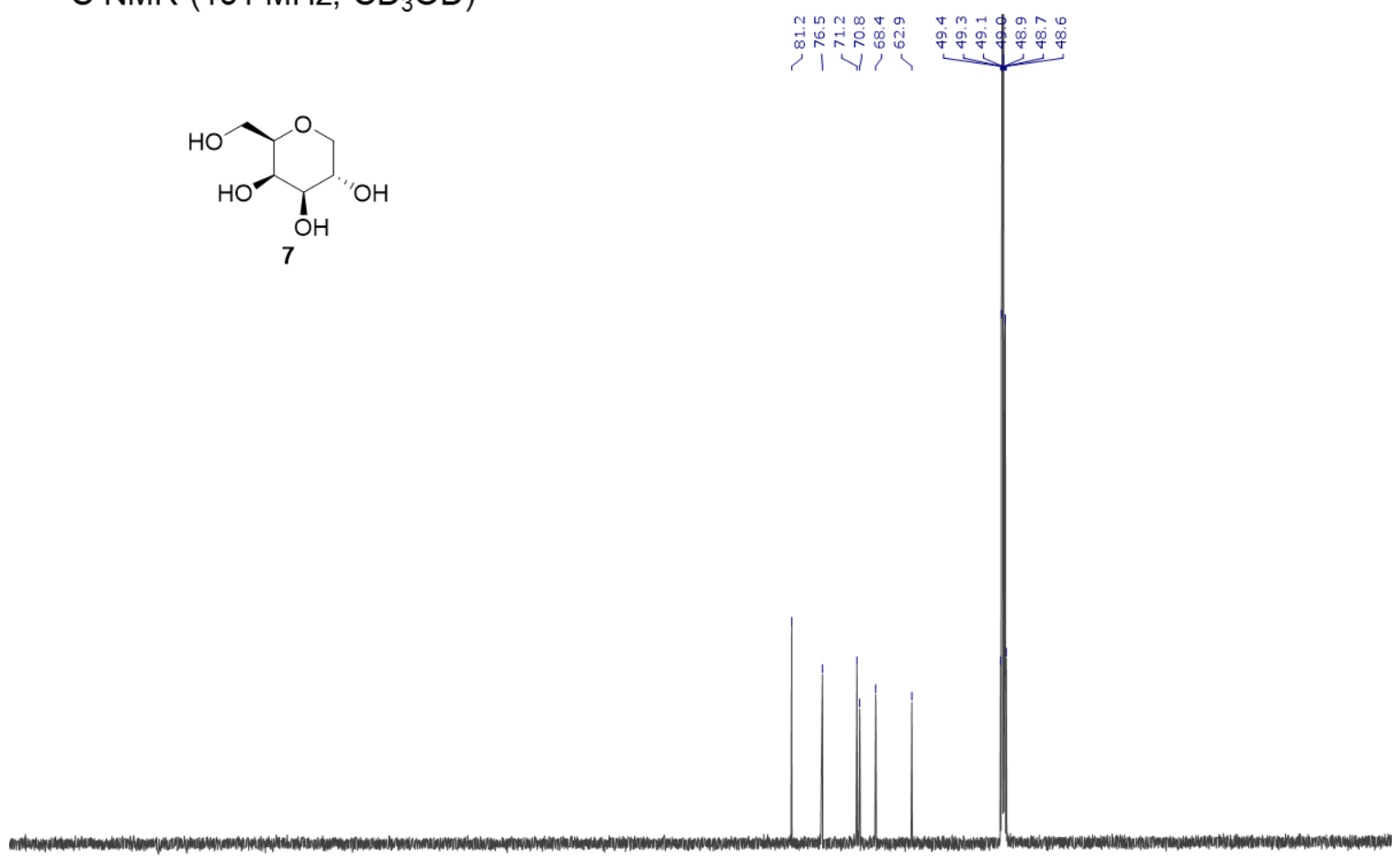

$\begin{array}{llllllllll}190 & 180 & 170 & 160 & 150 & 140 & 130 & 120 & 110 & 100 \\ \mathrm{f} 1(\mathrm{ppm}) & 90\end{array}$ 
${ }^{1} \mathrm{H}$ NMR $\left(600 \mathrm{MHz}, \mathrm{CD}_{3} \mathrm{OD}\right)$

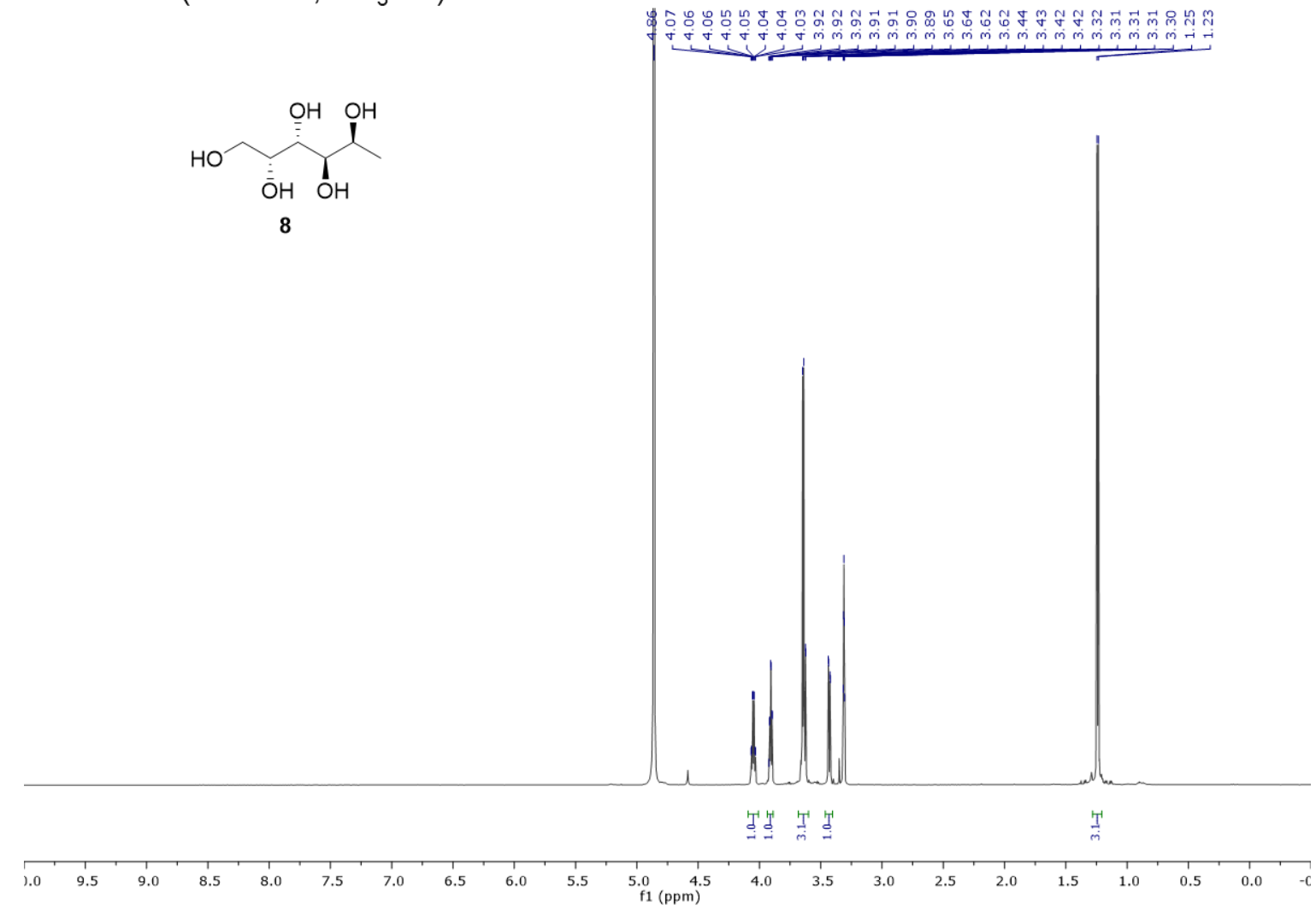

${ }^{13} \mathrm{C}$ NMR $\left(151 \mathrm{MHz}, \mathrm{CD}_{3} \mathrm{OD}\right)$
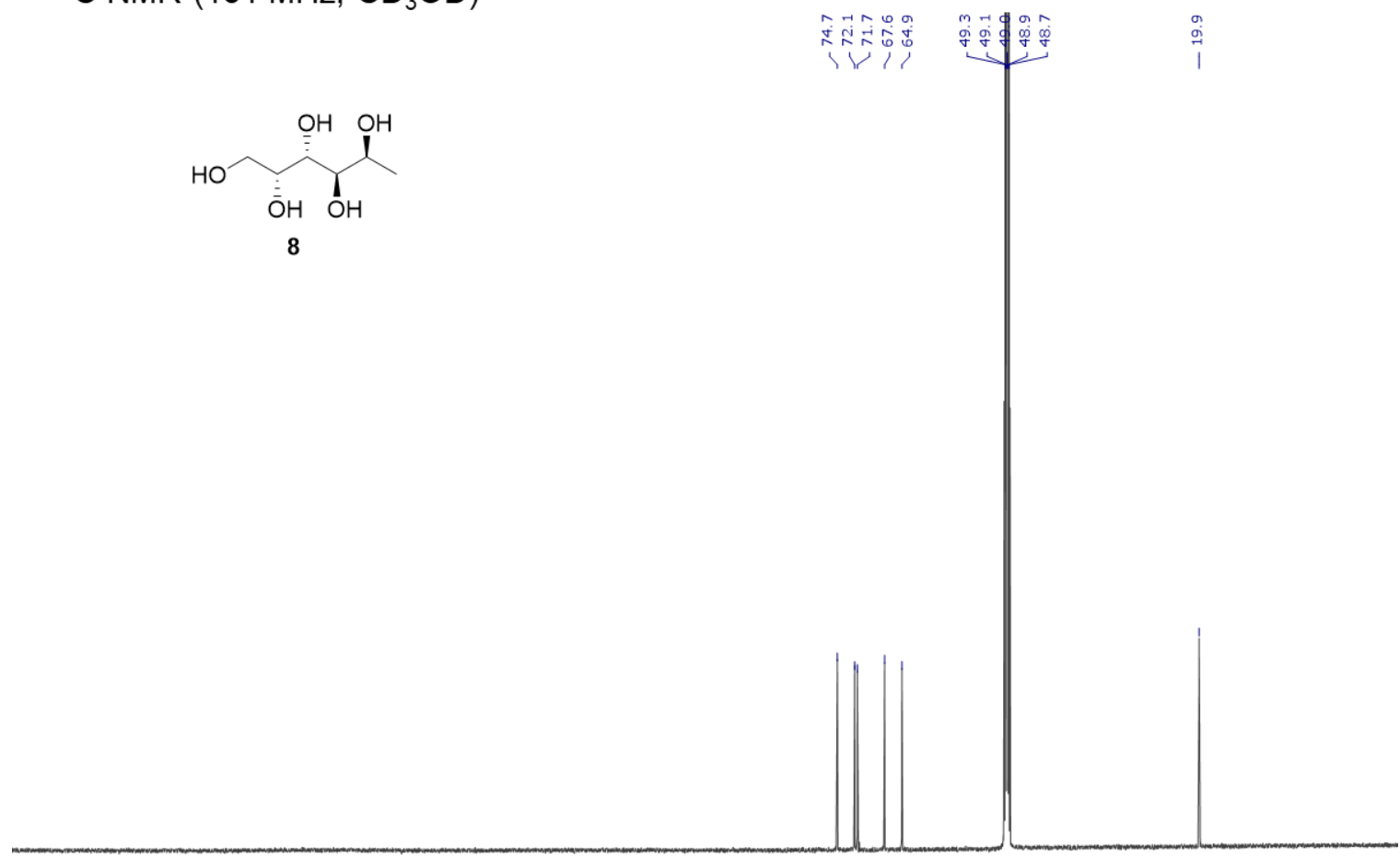

$\begin{array}{llllllllll}190 & 180 & 170 & 160 & 150 & 140 & 130 & 120 & 110 & 100 \\ \mathrm{f} 1(\mathrm{ppm}) & 90\end{array}$ 
${ }^{1} \mathrm{H}$ NMR $\left(600 \mathrm{MHz}, \mathrm{CD}_{3} \mathrm{OD}\right)$

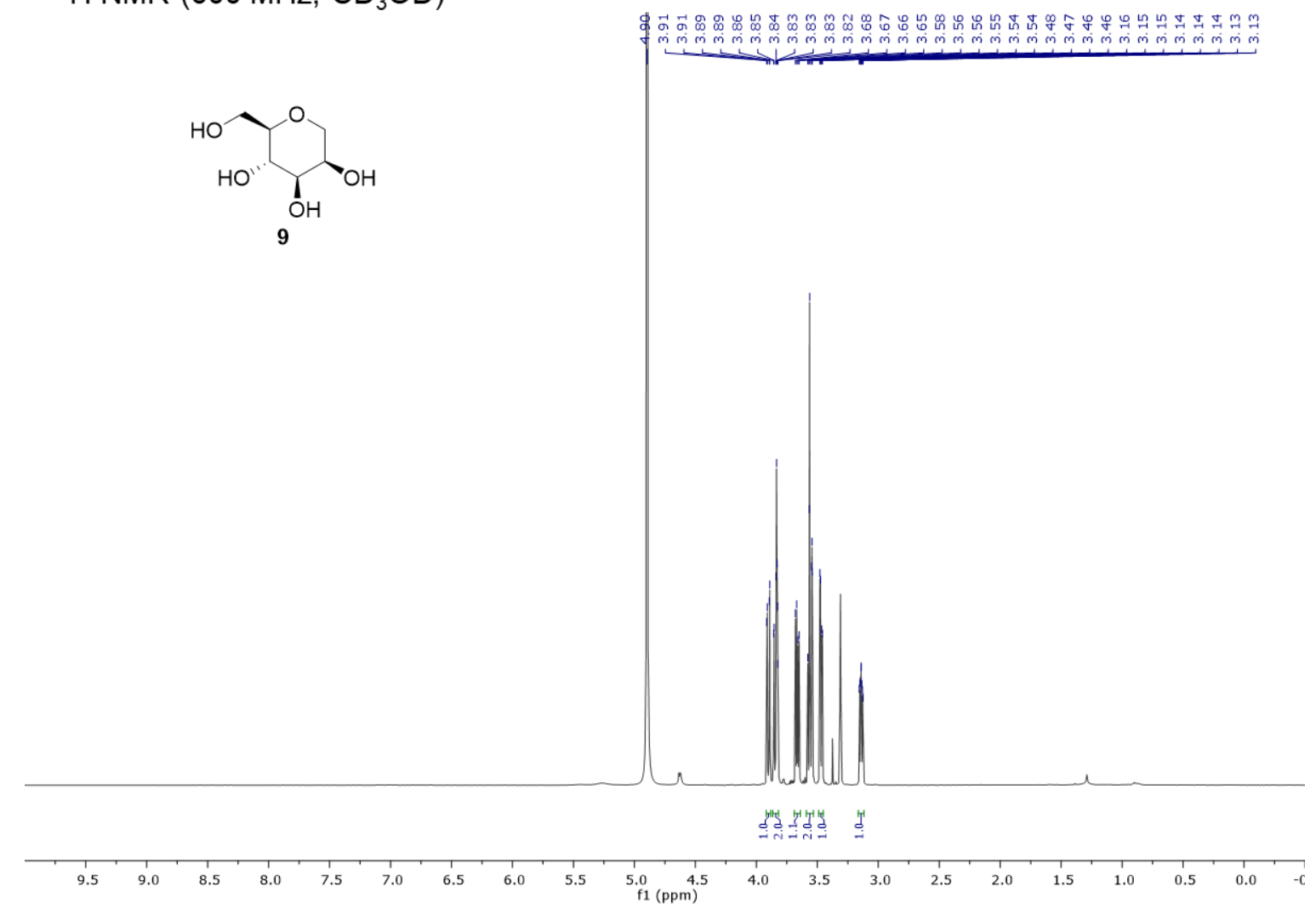

${ }^{13} \mathrm{C}$ NMR $\left(151 \mathrm{MHz}, \mathrm{CD}_{3} \mathrm{OD}\right)$
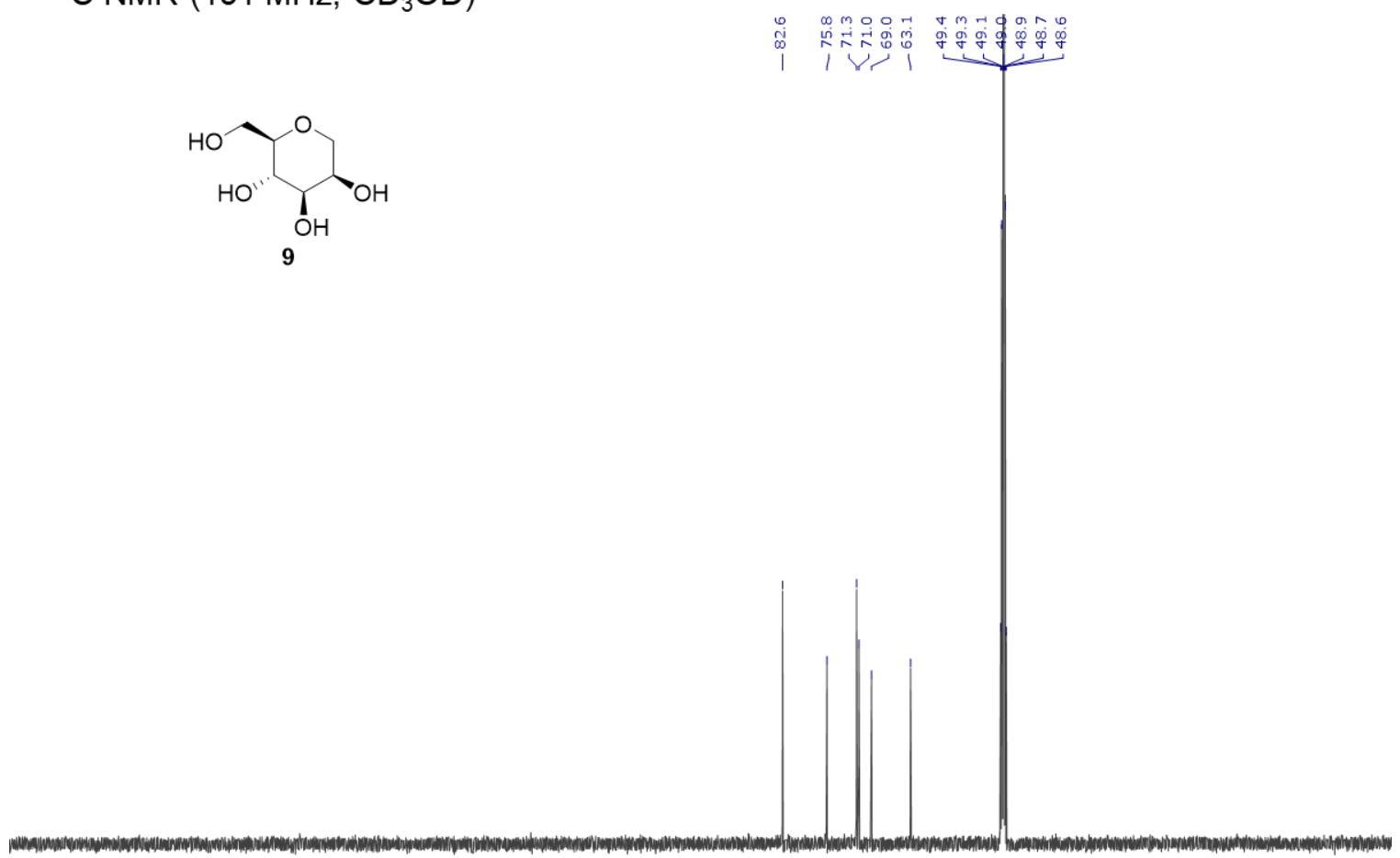

\begin{tabular}{lllllllllllllllllllllllllll}
\hline & 190 & 180 & 170 & 160 & 150 & 140 & 130 & 120 & 110 & 100 & 90 & 80 & 70 & 60 & 50 & 40 & 30 & 20 & 10 & 0 & -1
\end{tabular} 
${ }^{1} \mathrm{H}$ NMR $\left(600 \mathrm{MHz}, \mathrm{CD}_{3} \mathrm{OD}\right)$

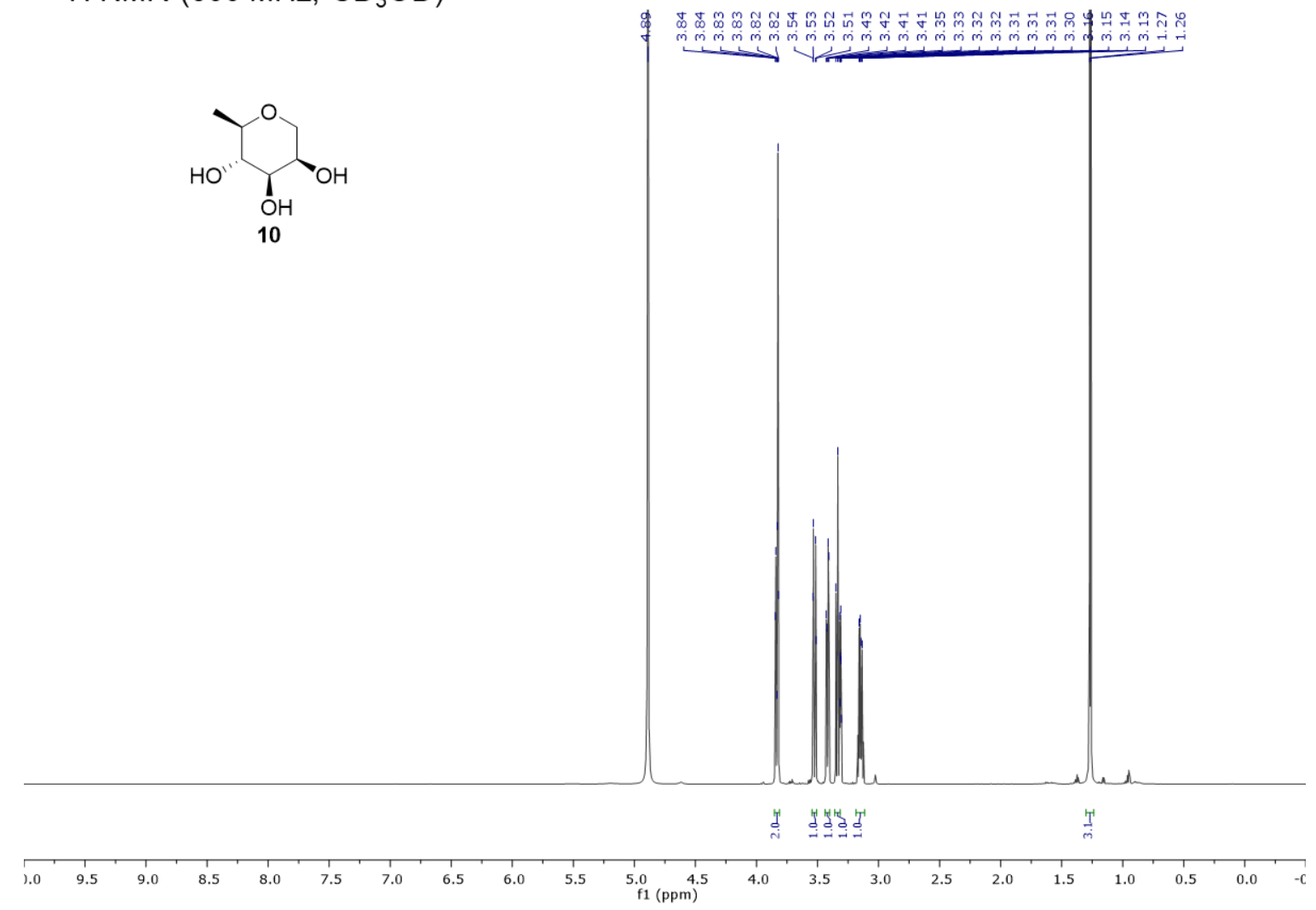

${ }^{13} \mathrm{C}$ NMR $\left(151 \mathrm{MHz}, \mathrm{CD}_{3} \mathrm{OD}\right)$
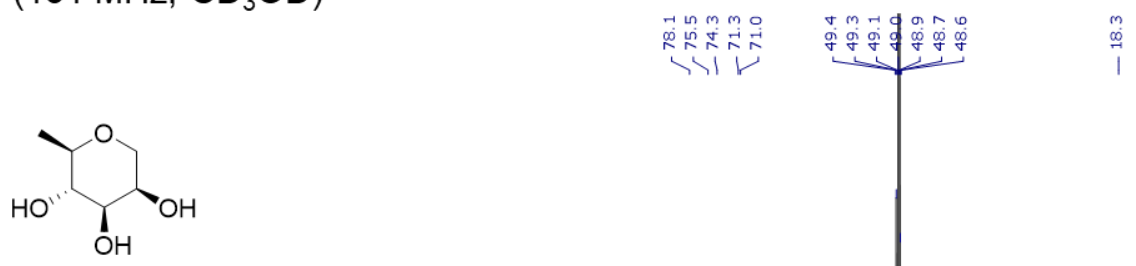

10

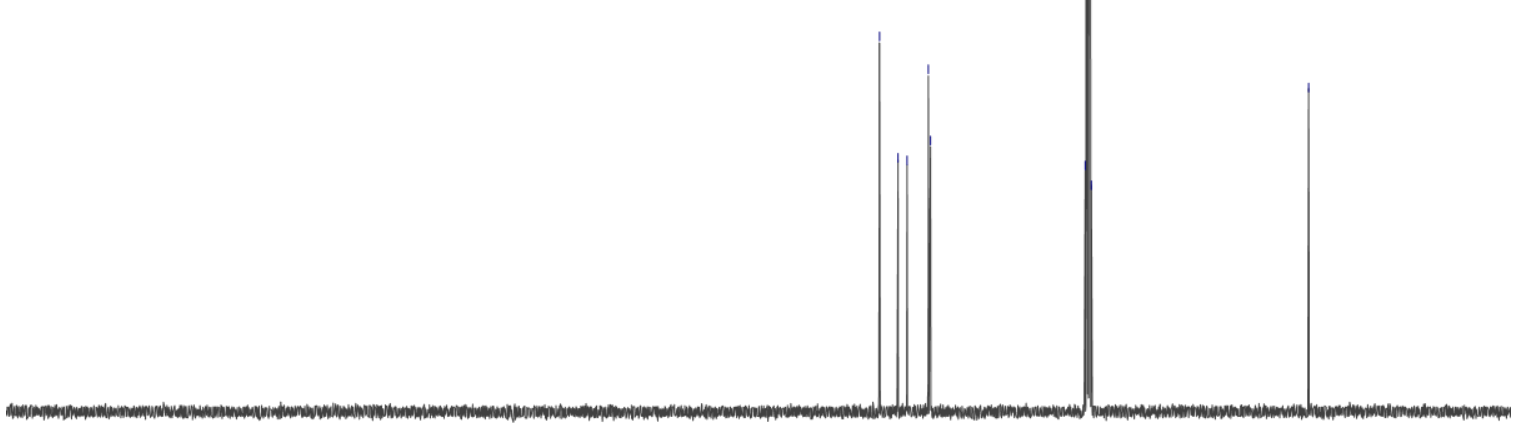


${ }^{1} \mathrm{H}$ NMR $\left(600 \mathrm{MHz}, \mathrm{CD}_{3} \mathrm{OD}\right)$

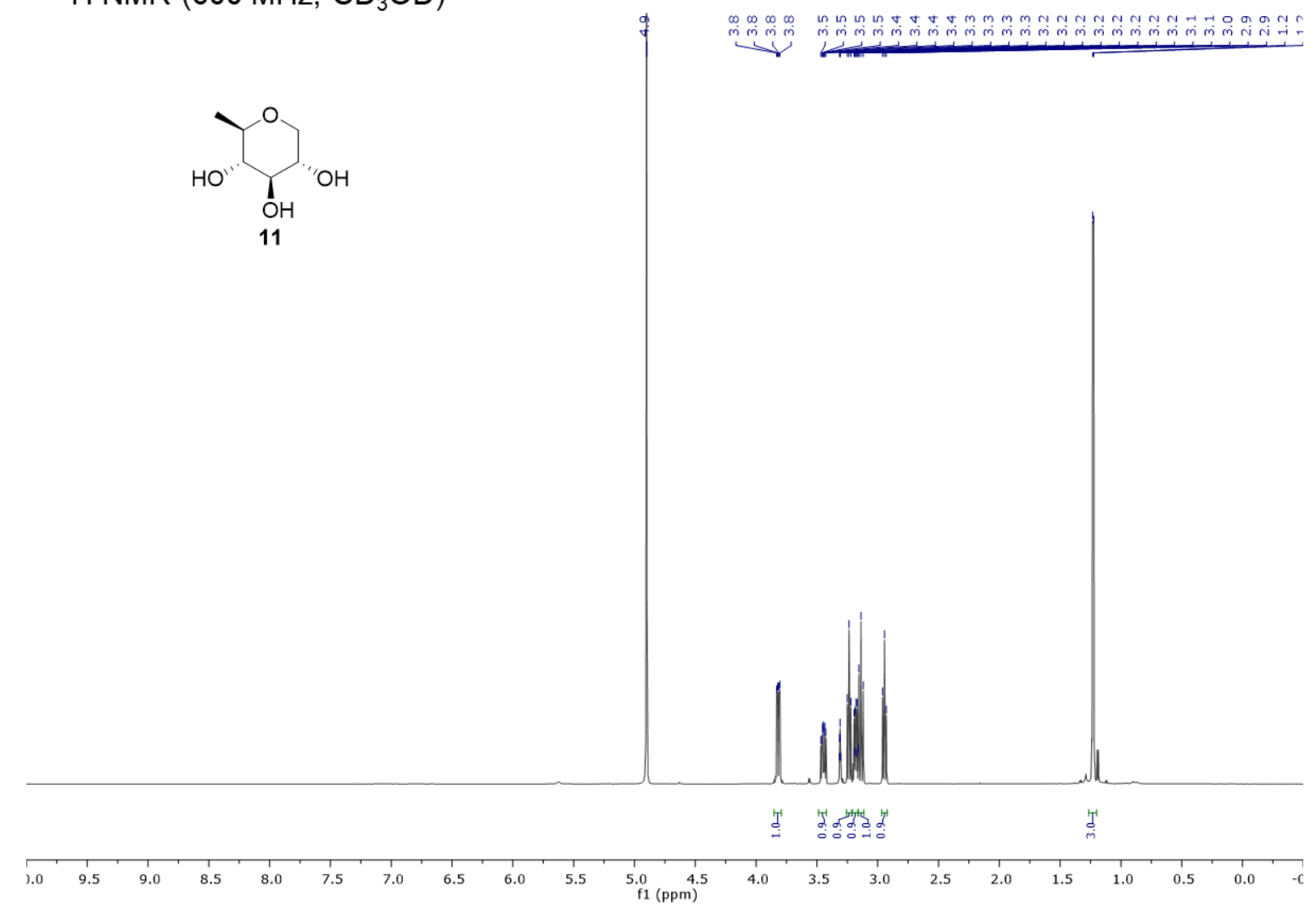

${ }^{13} \mathrm{C}$ NMR $\left(151 \mathrm{MHz}, \mathrm{CD}_{3} \mathrm{OD}\right)$
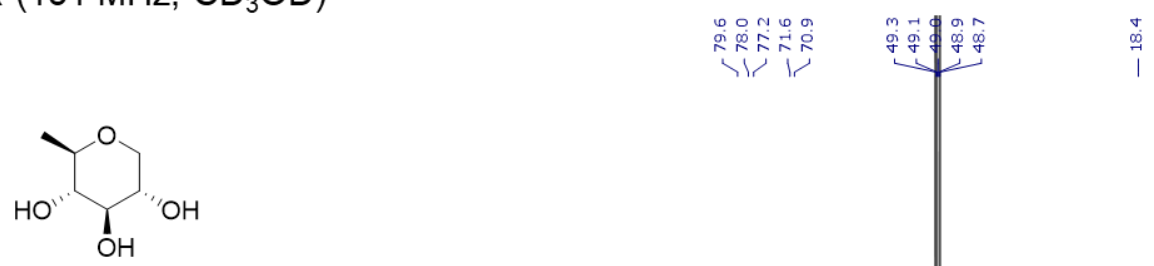

11

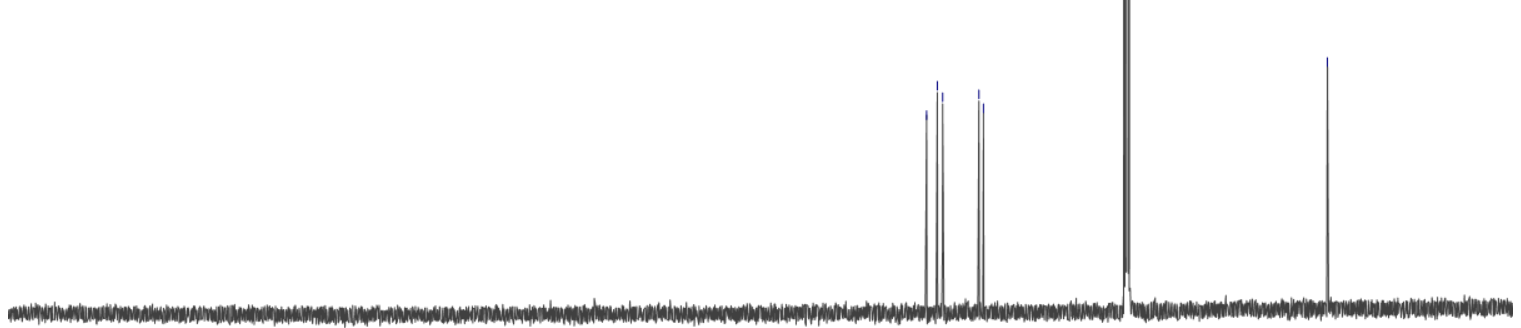

$\begin{array}{lllllllllllll}20 & 210 & 200 & 190 & 180 & 170 & 160 & 150 & 140 & 130 & 120 & 110 & 100\end{array}$ 
${ }^{1} \mathrm{H}$ NMR $\left(600 \mathrm{MHz}, \mathrm{CD}_{3} \mathrm{OD}\right)$

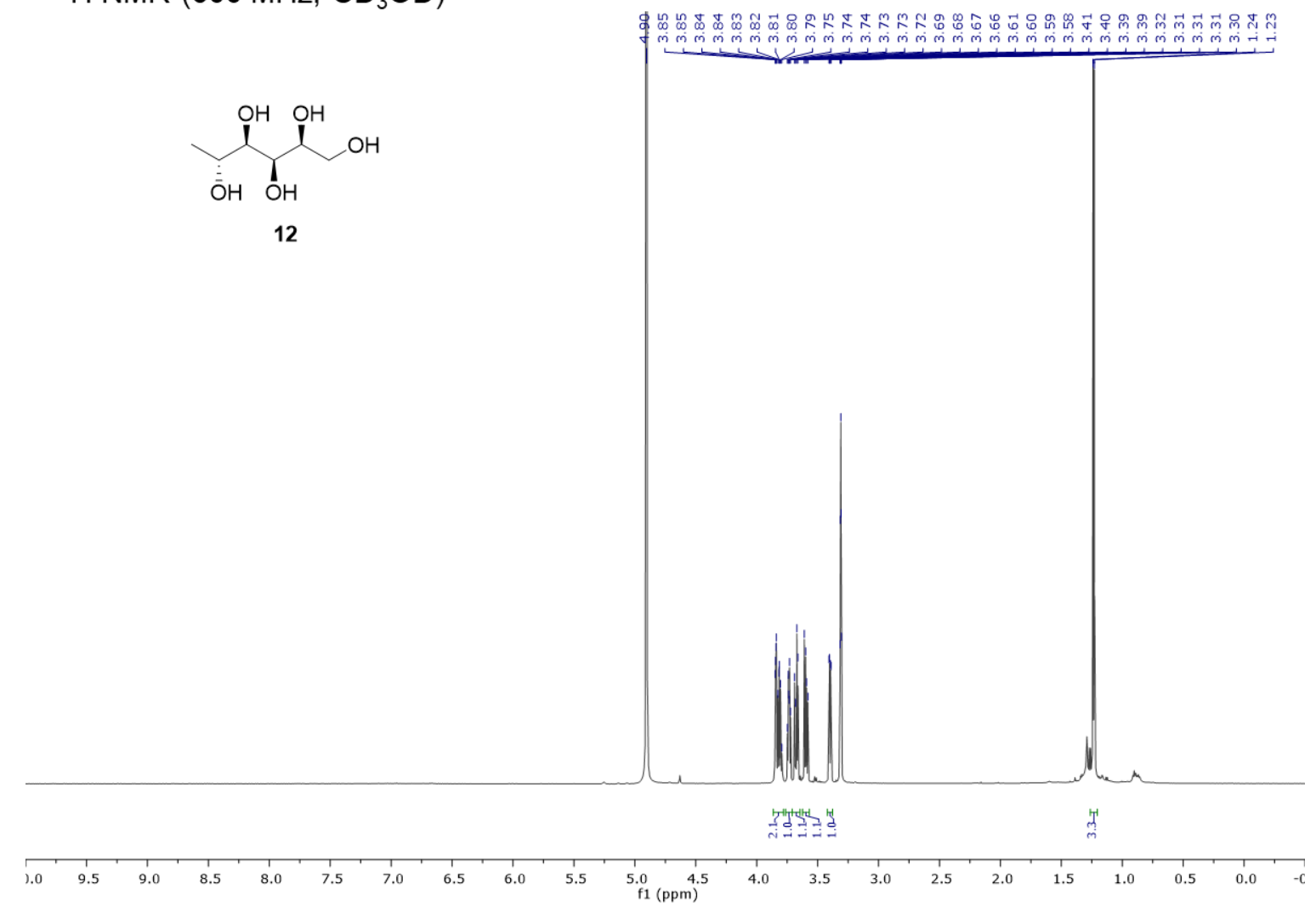

${ }^{13} \mathrm{C}$ NMR $\left(151 \mathrm{MHz}, \mathrm{CD}_{3} \mathrm{OD}\right)$
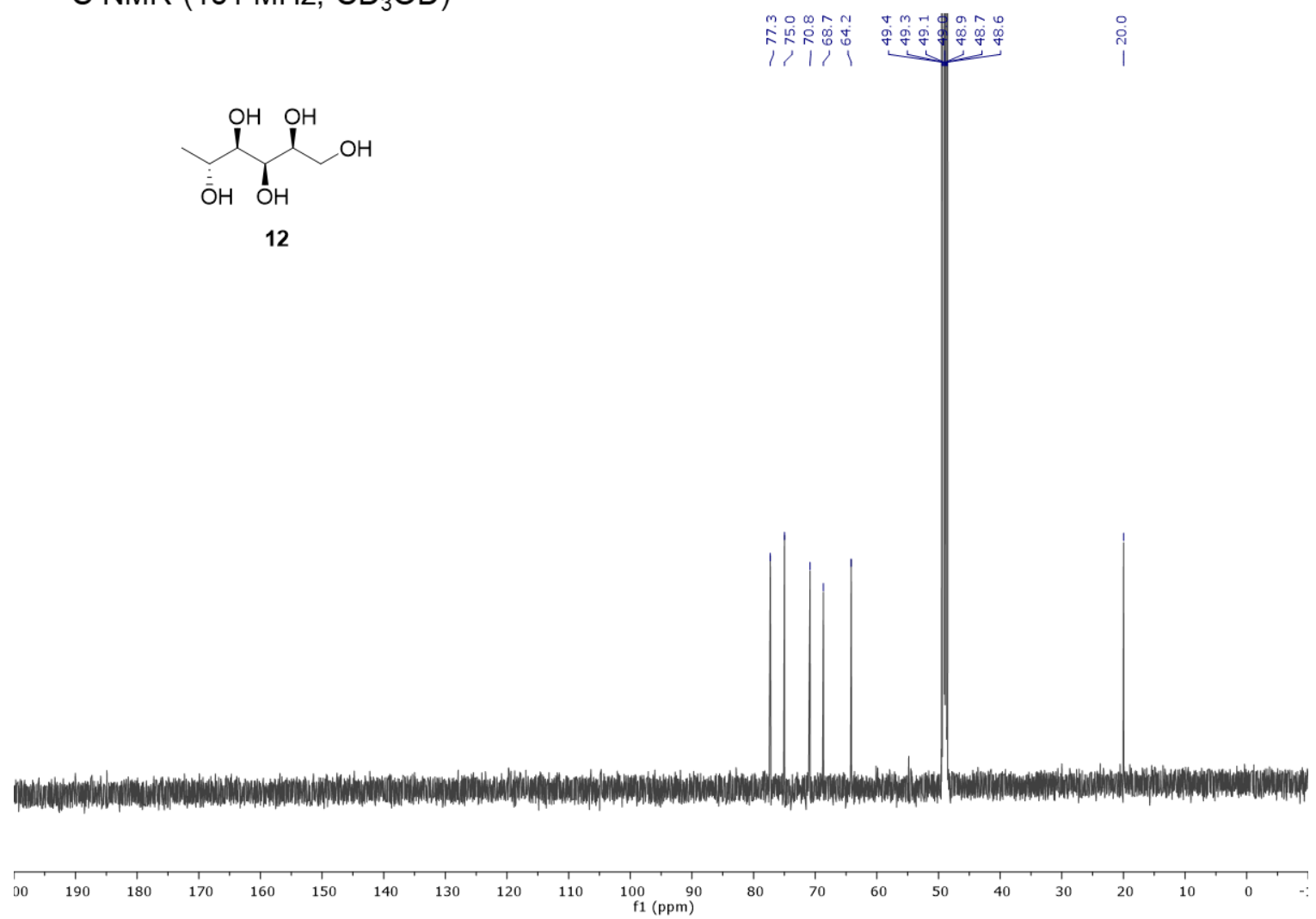
${ }^{1} \mathrm{H}$ NMR $\left(600 \mathrm{MHz}, \mathrm{CD}_{3} \mathrm{OD}\right)$

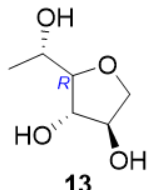

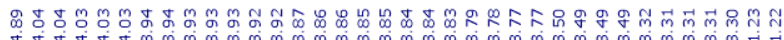

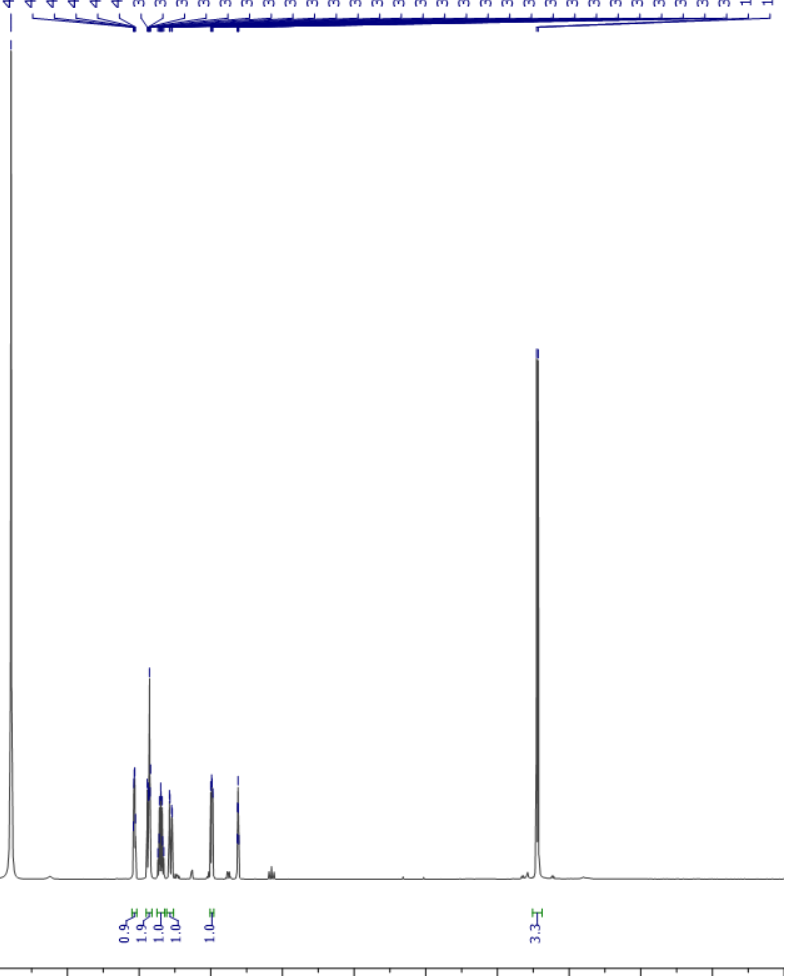

\begin{tabular}{rrrrrrrrrrr}
\hline .0 & 9.5 & 9.0 & 8.5 & 8.0 & 7.5 & 7.0 & 6.5 & 6.0 & 5.5 & $5.0 \quad 4$. \\
& & & & & & & & & & \\
$f 1(\mathrm{ppm})$
\end{tabular}

${ }^{13} \mathrm{C}$ NMR (151 MHz, $\left.\mathrm{CD}_{3} \mathrm{OD}\right)$
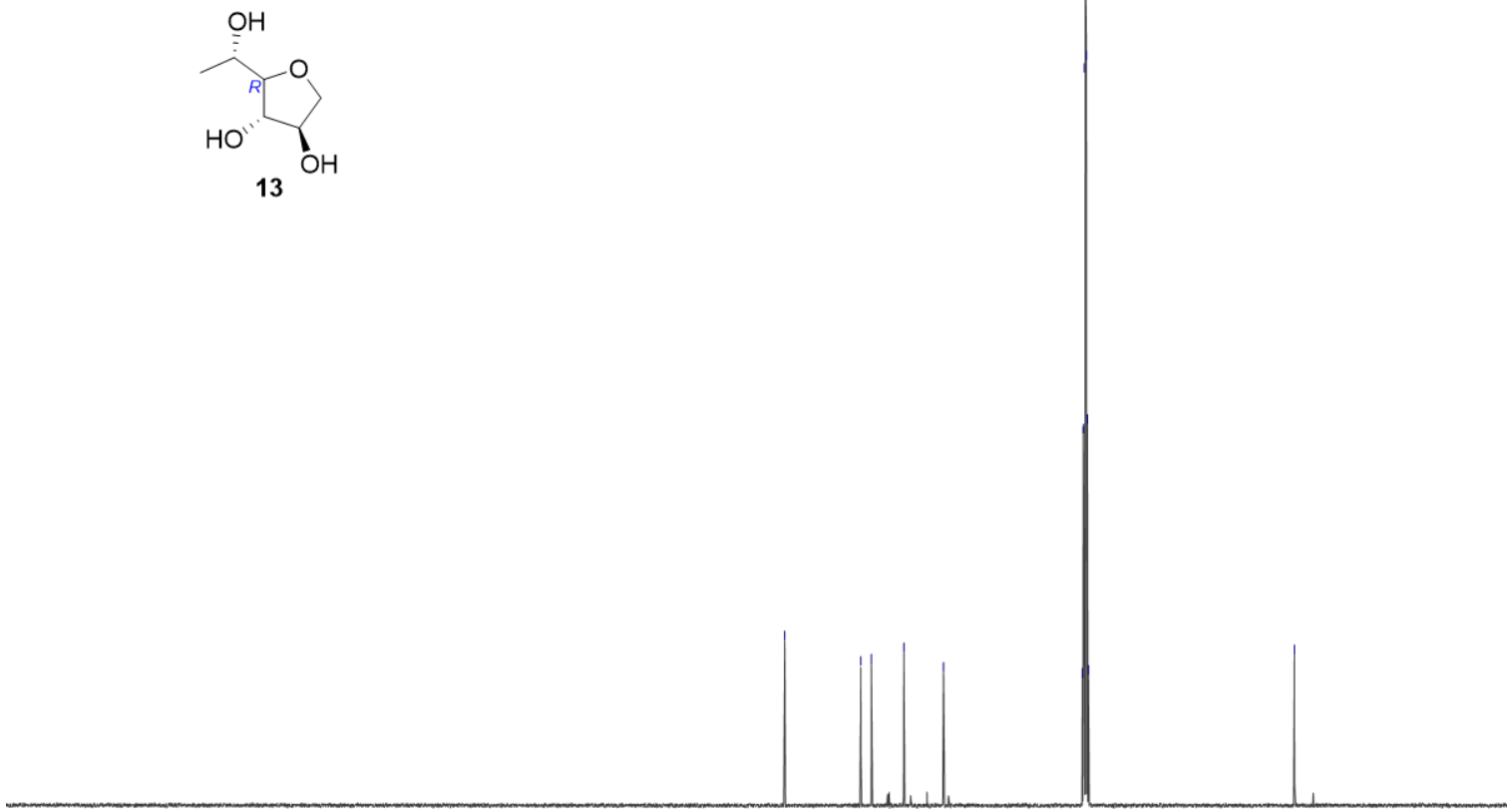

$\begin{array}{lllllllllll}00 & 190 & 180 & 170 & 160 & 150 & 140 & 130 & 120 & 110 & \begin{array}{c}100 \\ \mathrm{f} 1(\mathrm{ppm})\end{array}\end{array}$ 


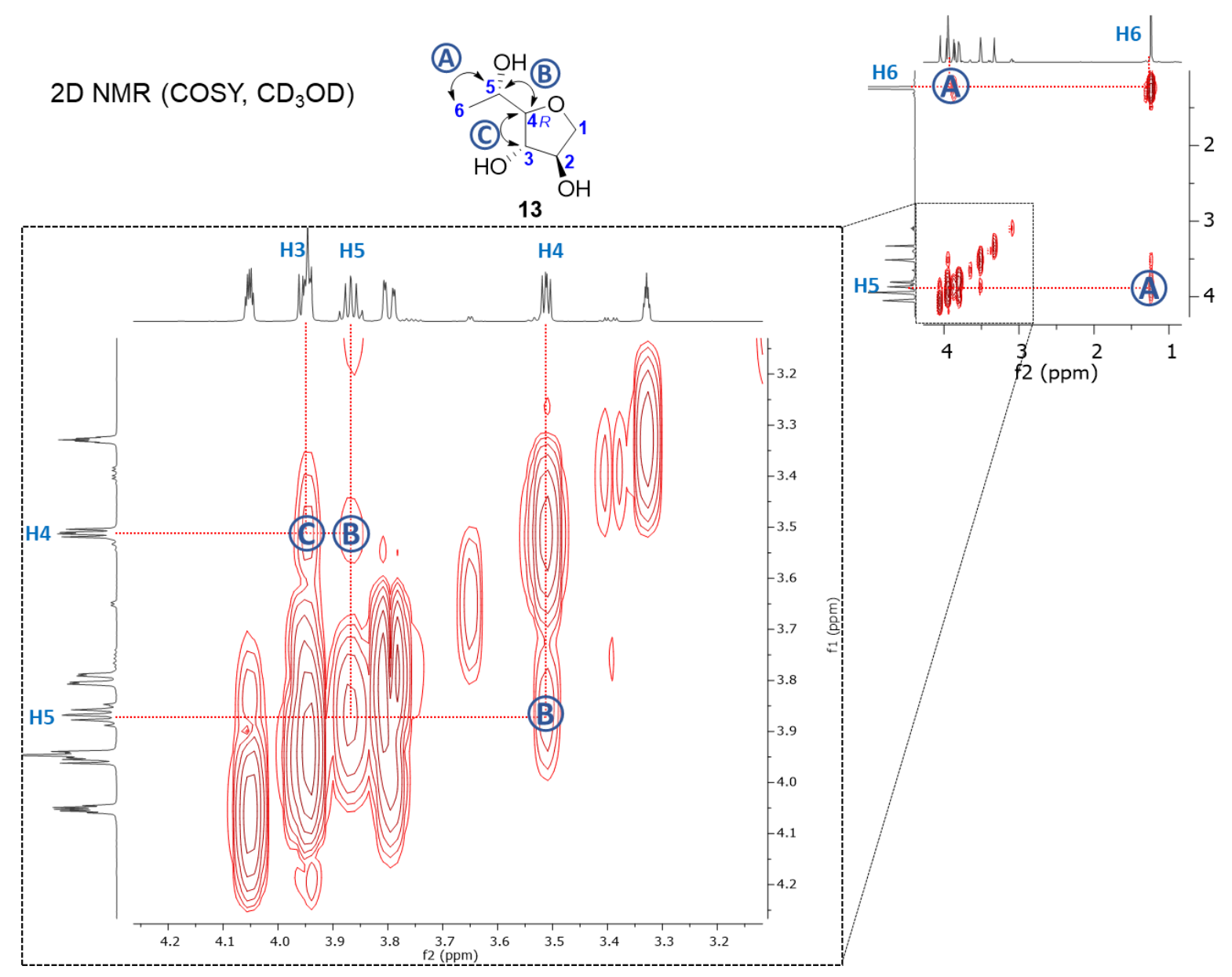




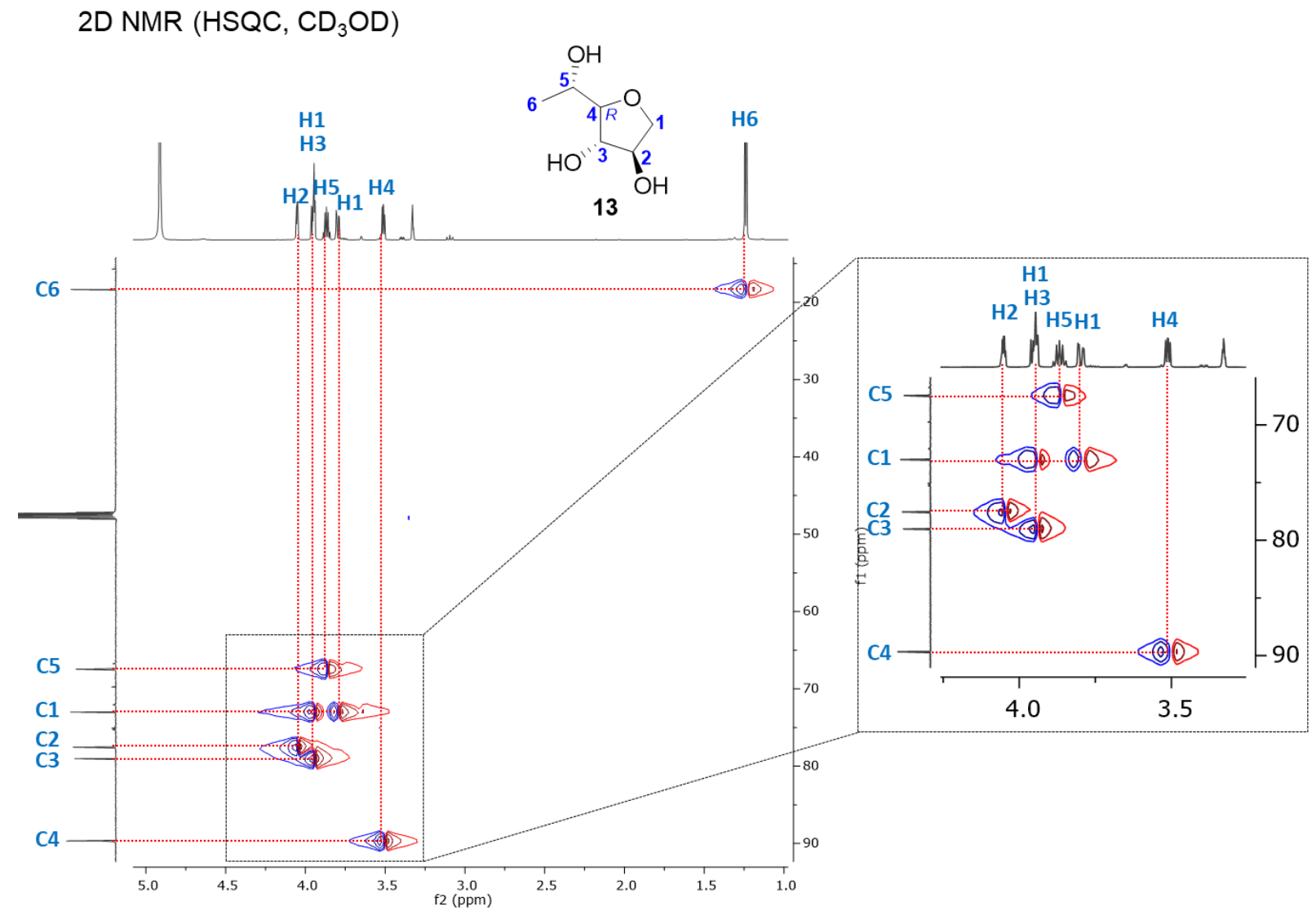


2D NMR ( $\left.\mathrm{HMBC}, \mathrm{CD}_{3} \mathrm{OD}\right)$

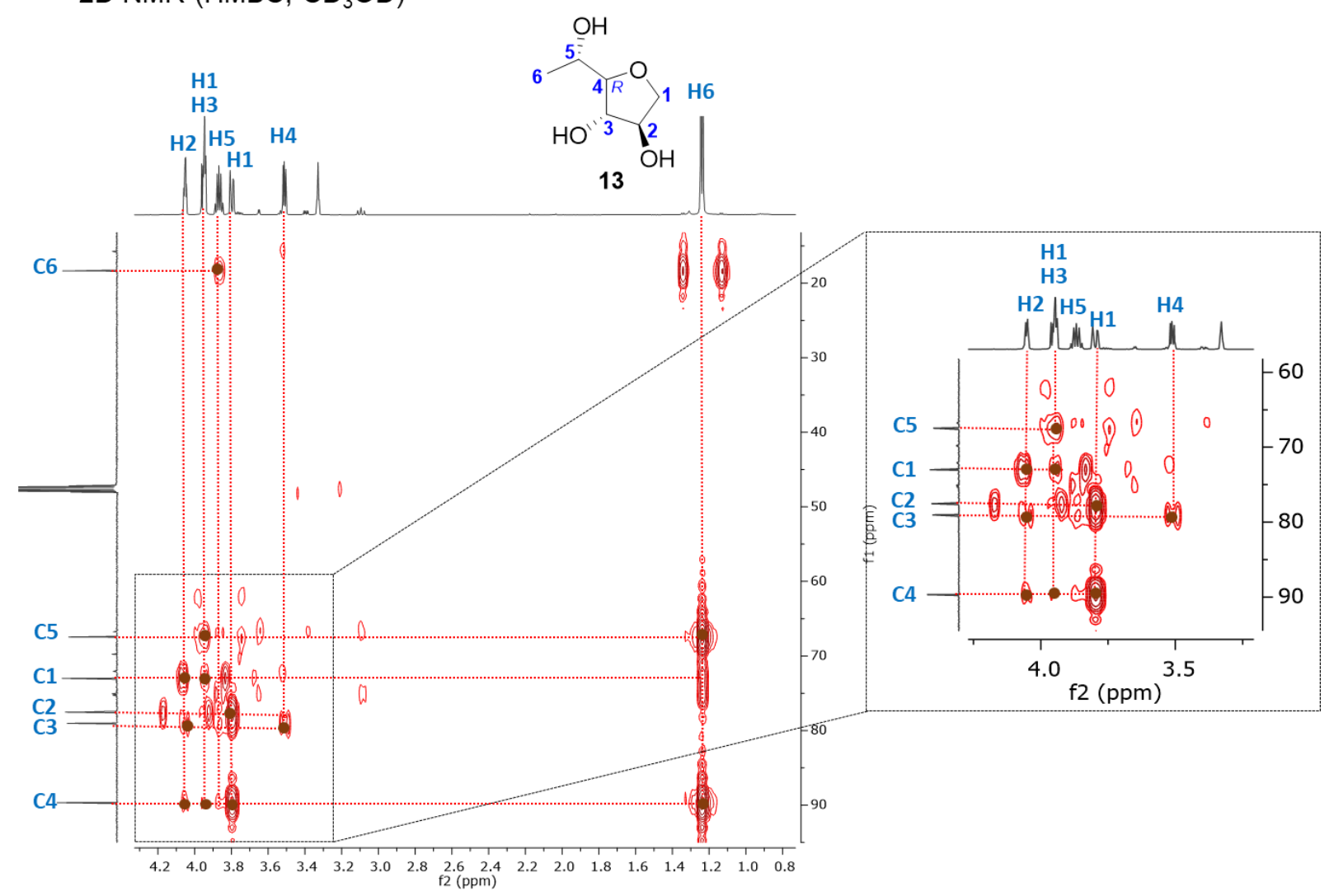


${ }^{1} \mathrm{H}$ NMR $\left(600 \mathrm{MHz}, \mathrm{CD}_{3} \mathrm{OD}\right)$

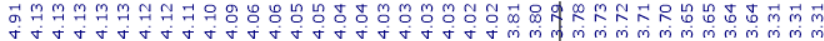

प्य

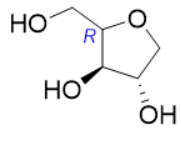

14

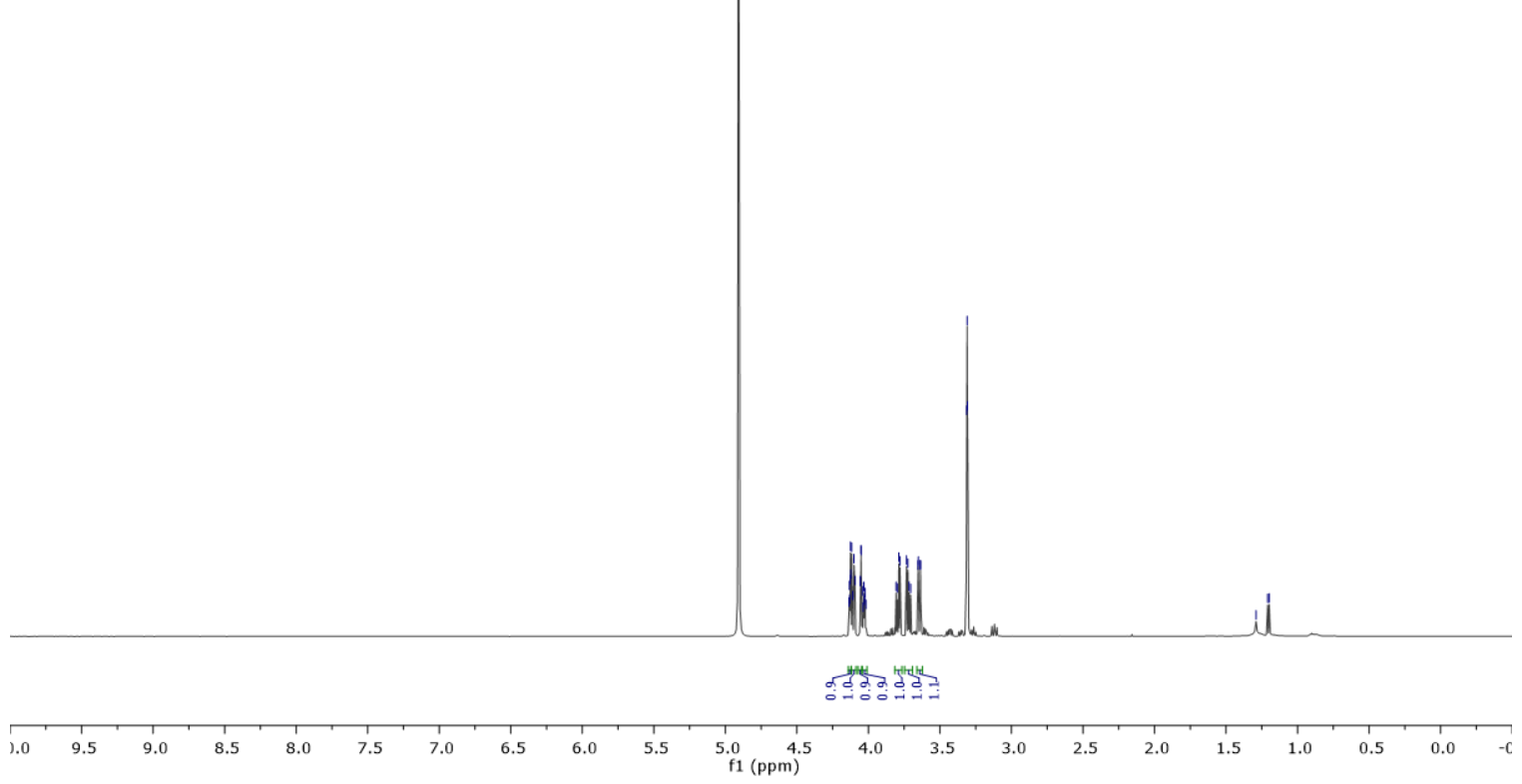

${ }^{13} \mathrm{C}$ NMR $\left(151 \mathrm{MHz}, \mathrm{CD}_{3} \mathrm{OD}\right)$
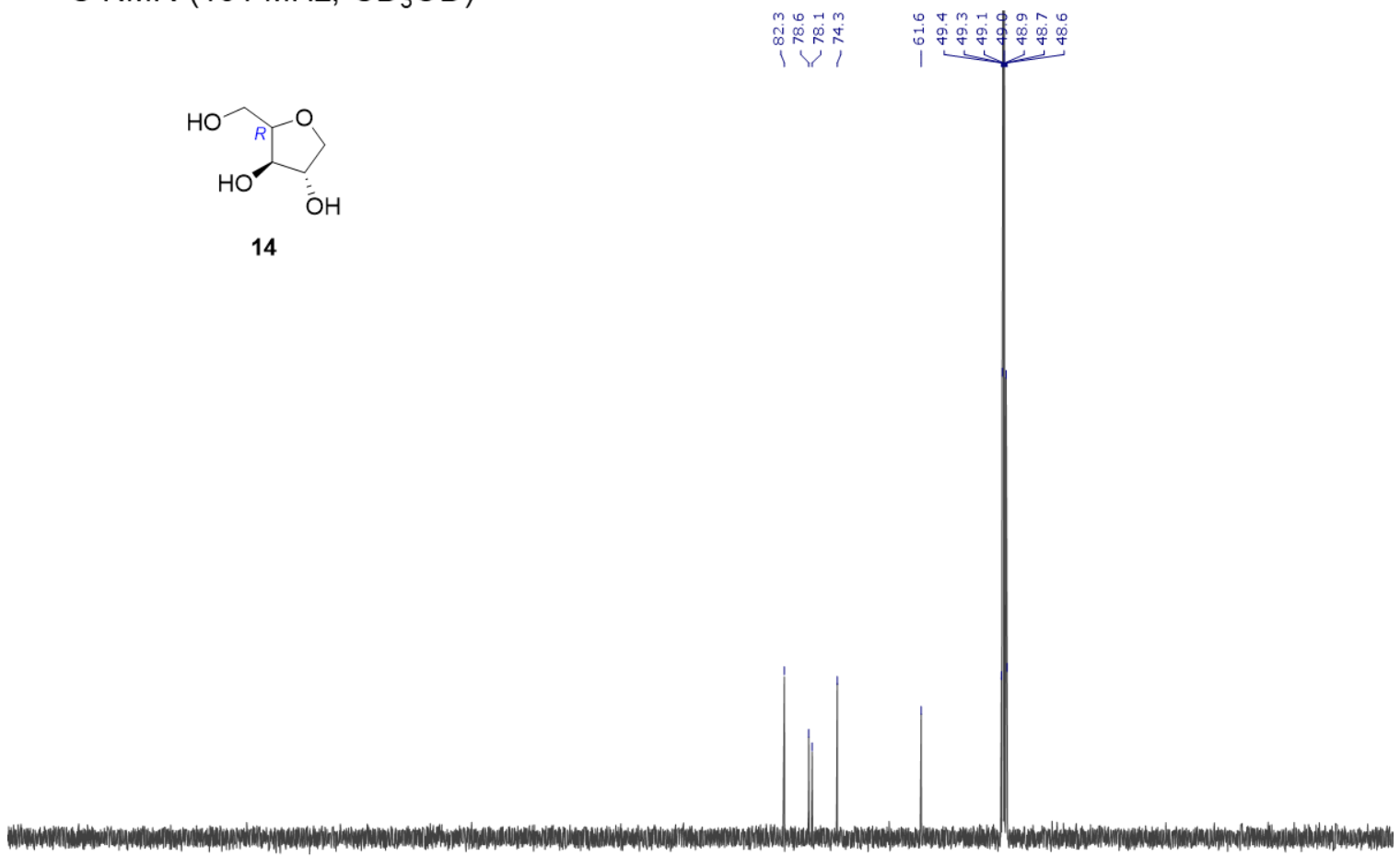

$\begin{array}{llllllllll}190 & 180 & 170 & 160 & 150 & 140 & 130 & 120 & 110 & 100 \\ \mathrm{f} 1(\mathrm{ppm}) & 90\end{array}$ 


\section{D NMR (COSY, $\left.\mathrm{CD}_{3} \mathrm{OD}\right)$}

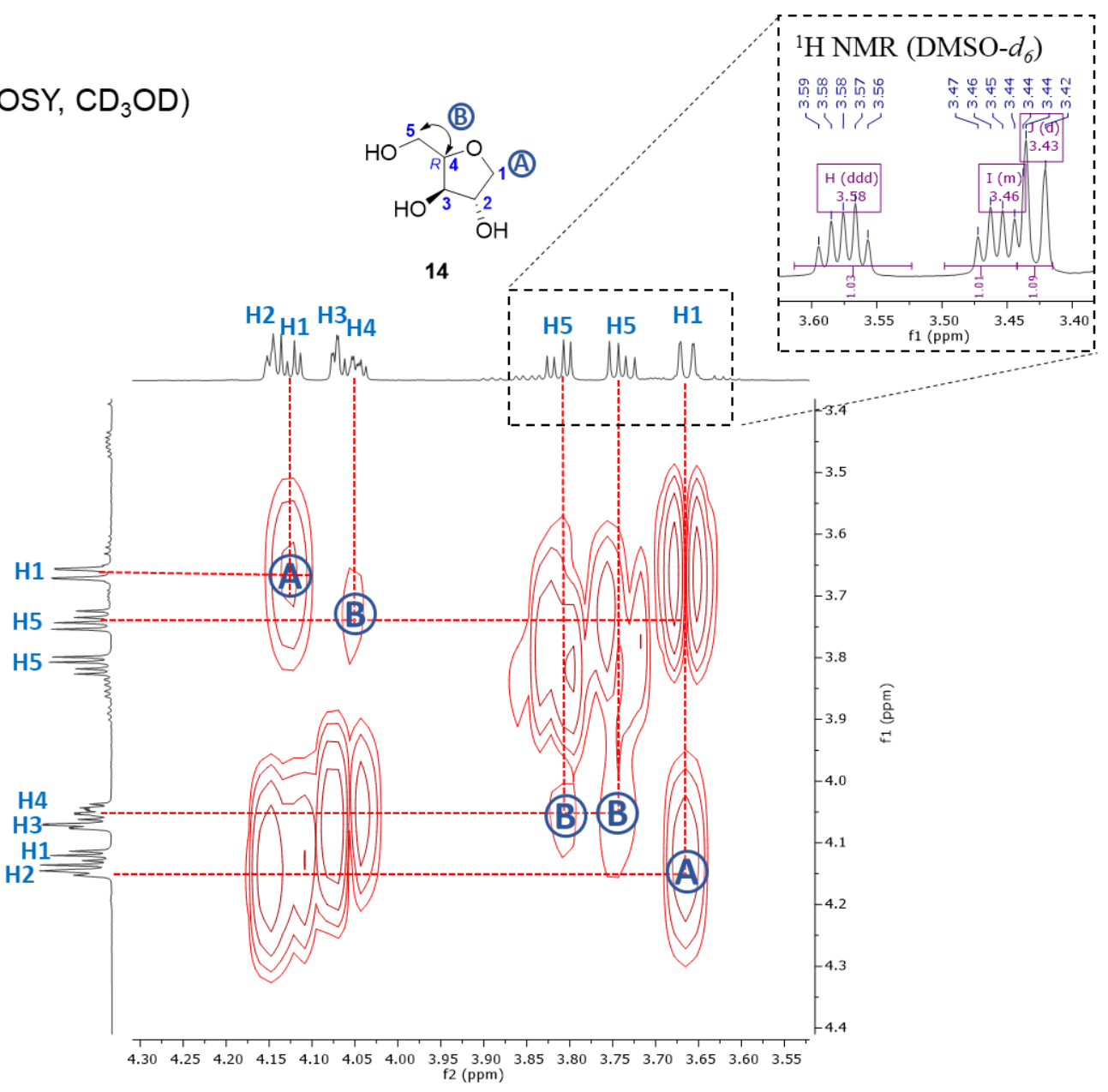


2D NMR (HSQC, $\left.\mathrm{CD}_{3} \mathrm{OD}\right)$

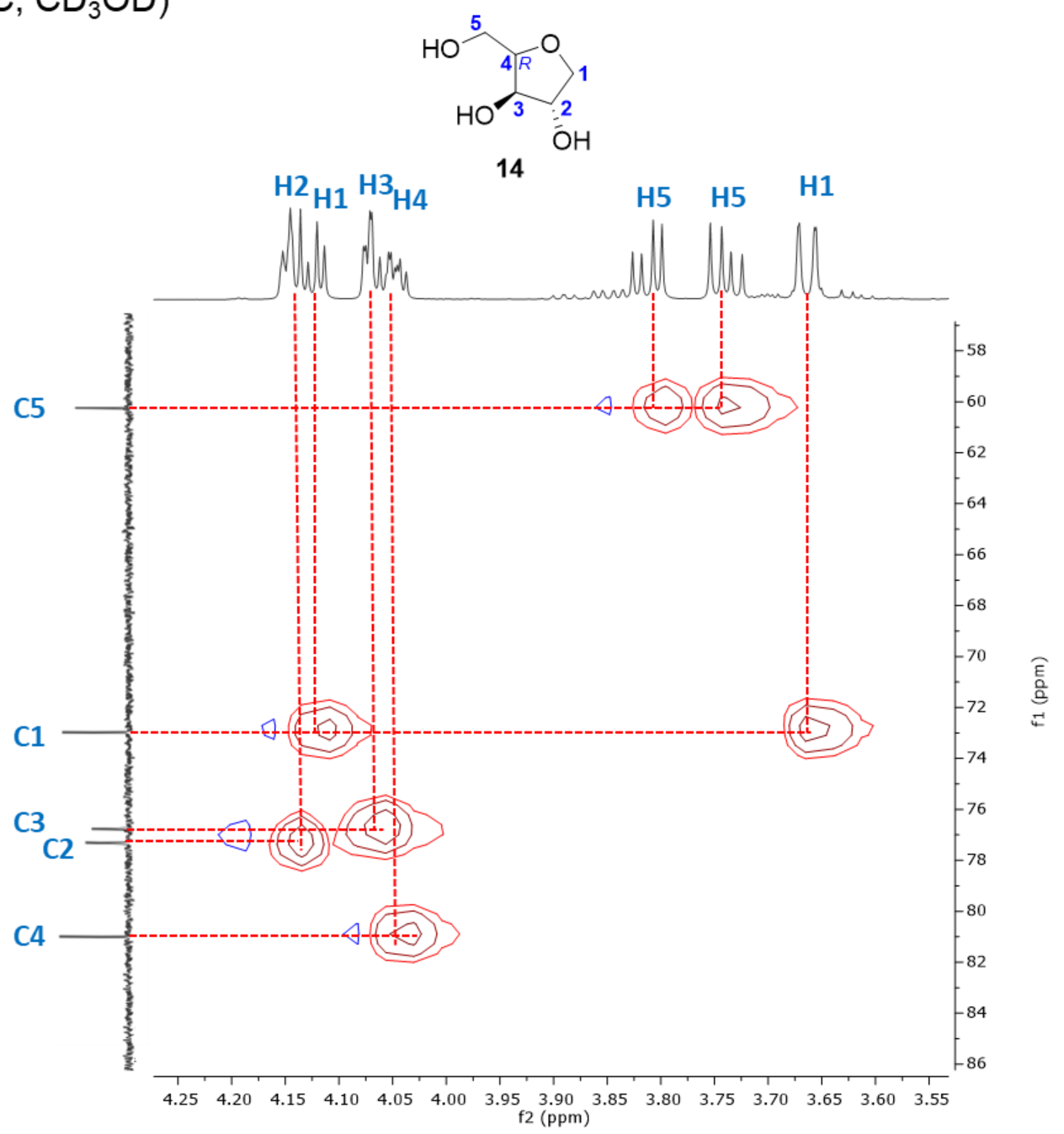


2D NMR (HMBC, $\left.\mathrm{CD}_{3} \mathrm{OD}\right)$

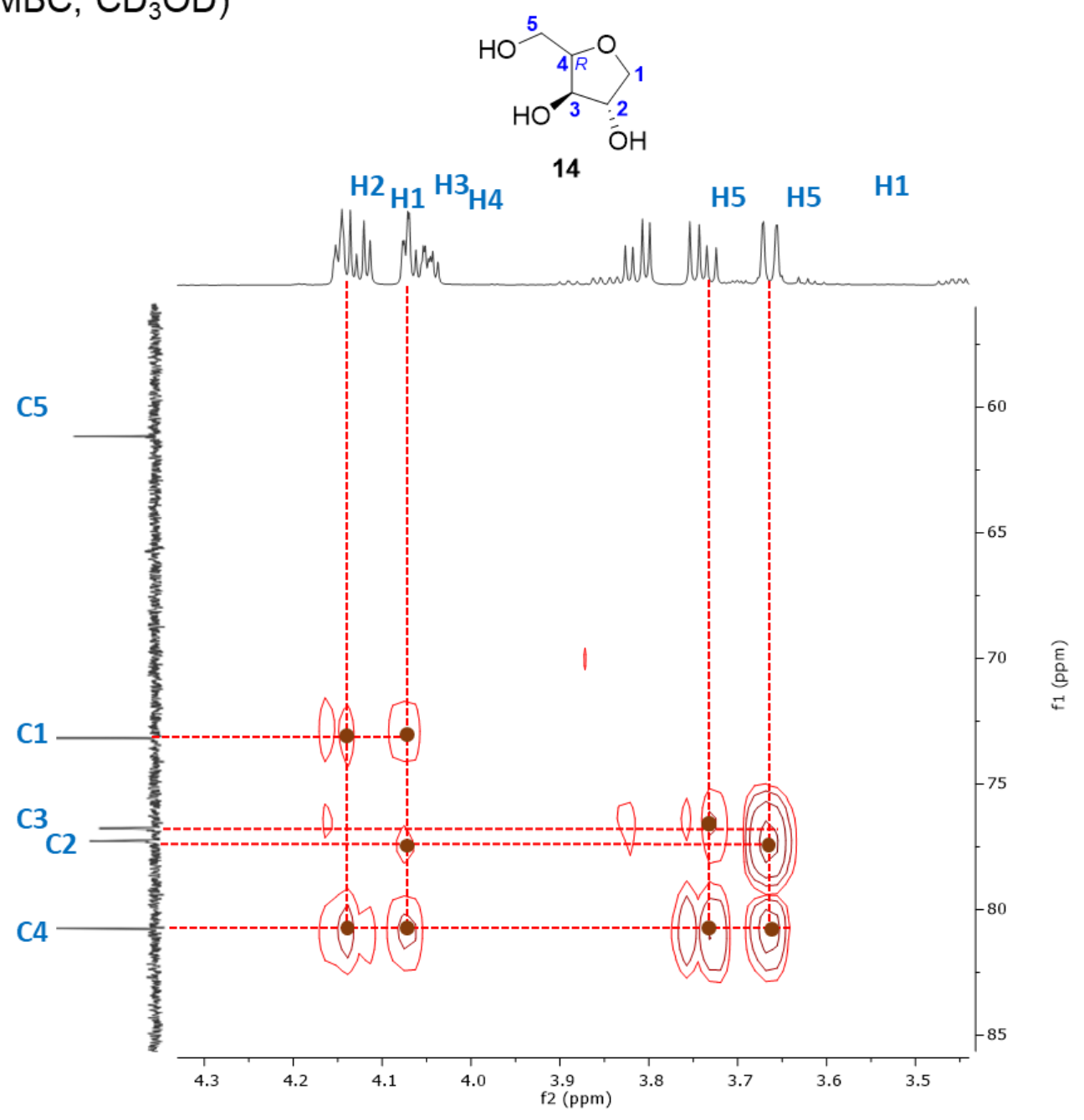


${ }^{1} \mathrm{H}$ NMR $\left(600 \mathrm{MHz}, \mathrm{CD}_{3} \mathrm{OD}\right)$

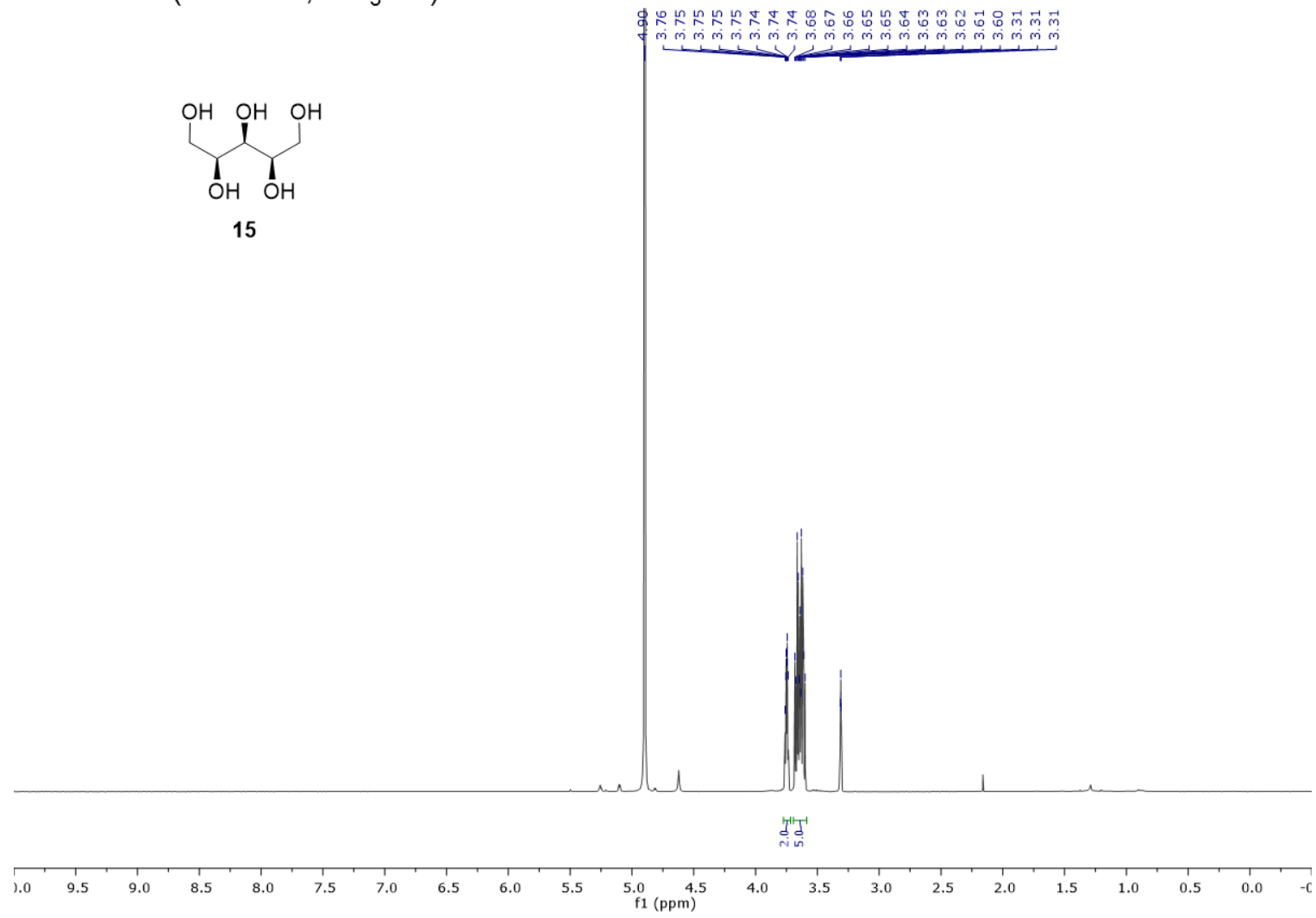

${ }^{13} \mathrm{C}$ NMR $\left(151 \mathrm{MHz}, \mathrm{CD}_{3} \mathrm{OD}\right)$

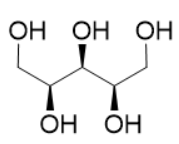

15

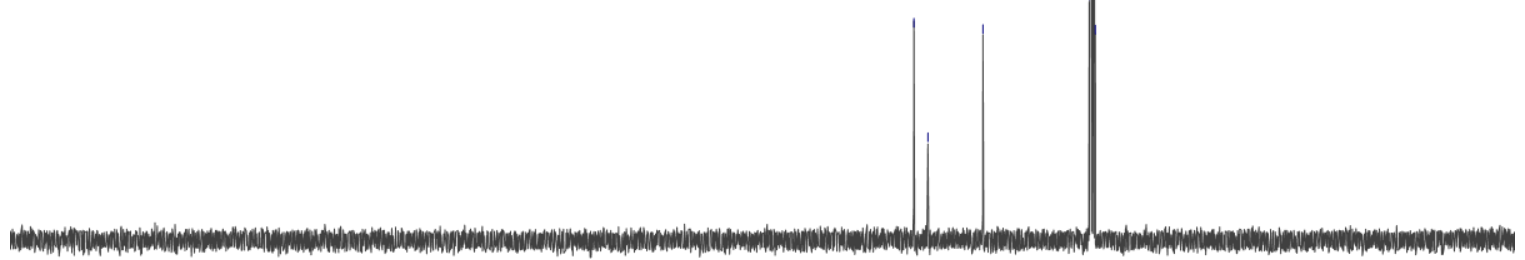


${ }^{1} \mathrm{H}$ NMR $\left(600 \mathrm{MHz}, \mathrm{CD}_{3} \mathrm{OD}\right)$

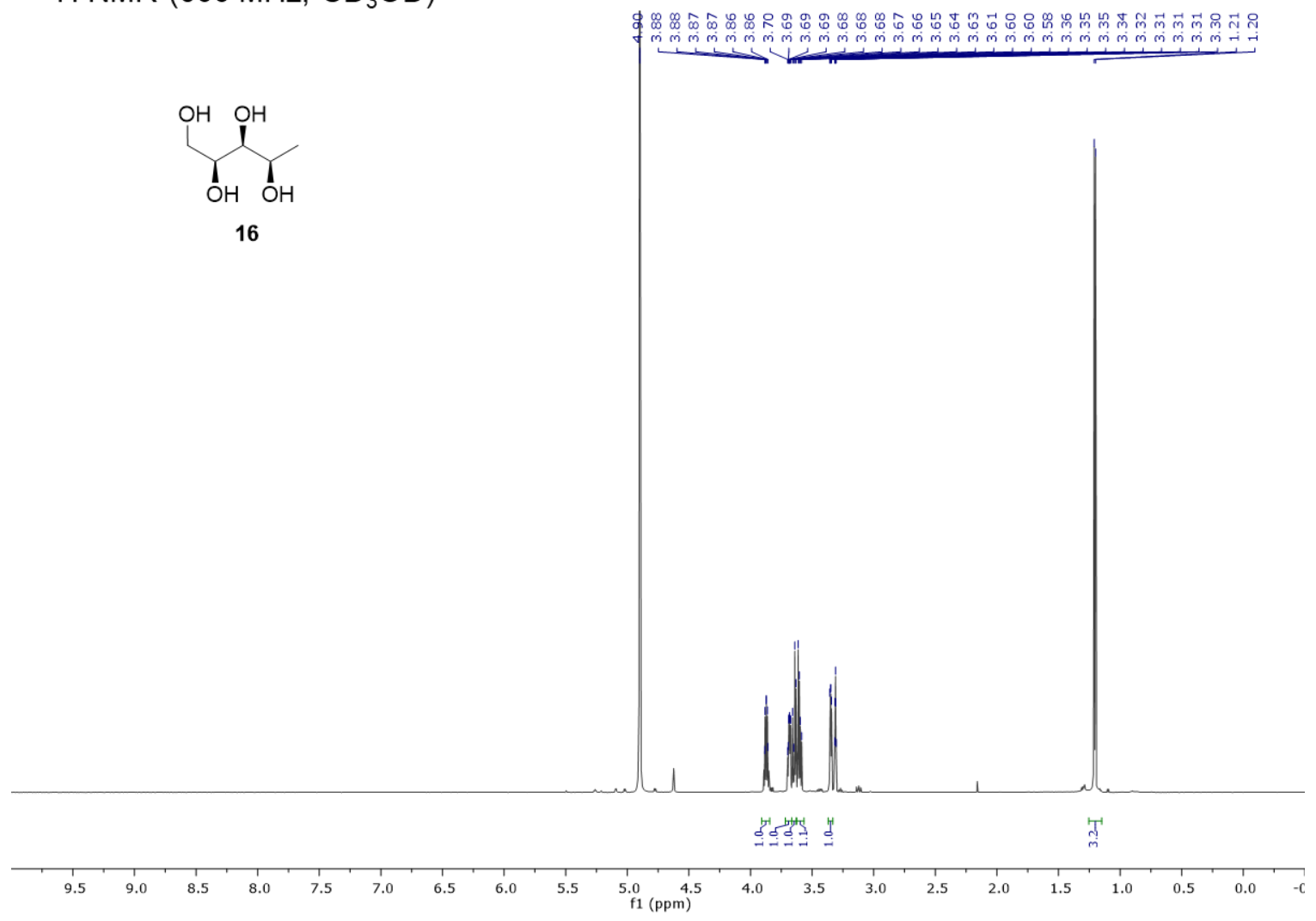

${ }^{13} \mathrm{C}$ NMR $\left(151 \mathrm{MHz}, \mathrm{CD}_{3} \mathrm{OD}\right)$

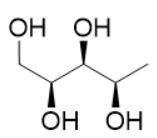

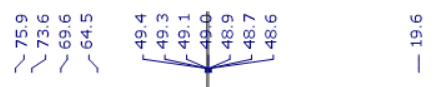

16

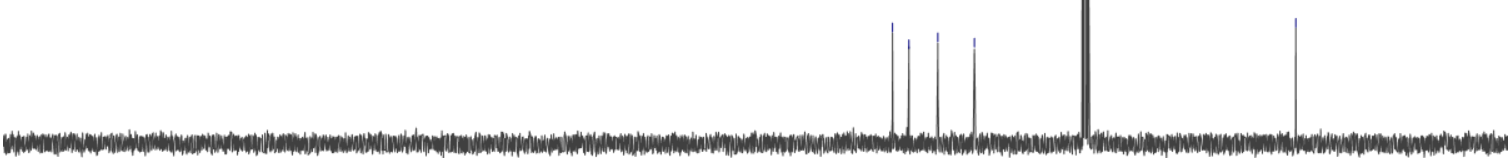

$\begin{array}{llllllllll}190 & 180 & 170 & 160 & 150 & 140 & 130 & 120 & 110 & 100 \\ \mathrm{f} 1(\mathrm{ppm}) & 90\end{array}$ 
2D NMR (COSY, $\left.\mathrm{CD}_{3} \mathrm{OD}\right)$

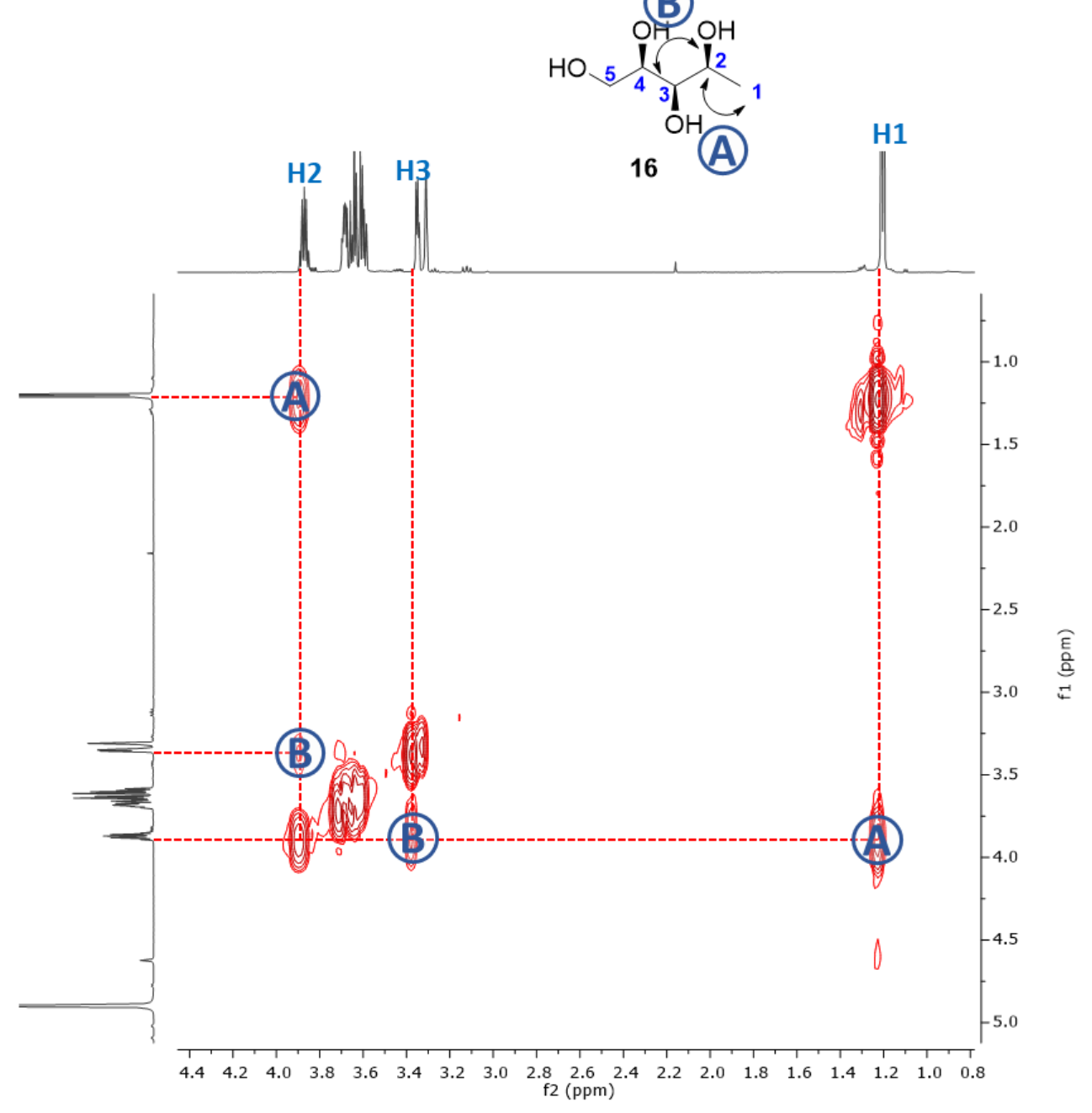


2D NMR ( $\left.\mathrm{HSQC}, \mathrm{CD}_{3} \mathrm{OD}\right)$

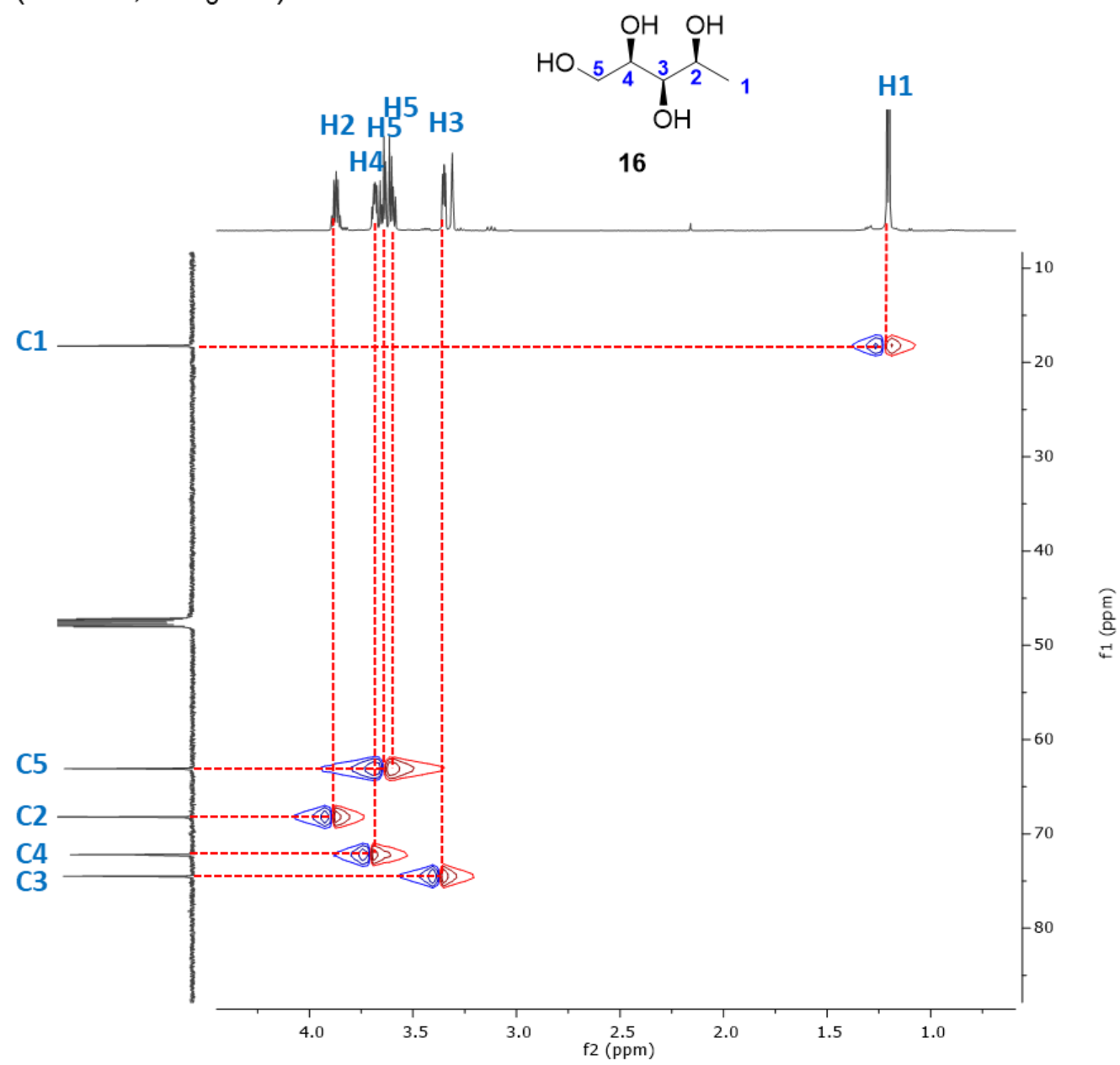




\section{D NMR ( $\left.\mathrm{HMBC}, \mathrm{CD}_{3} \mathrm{OD}\right)$}

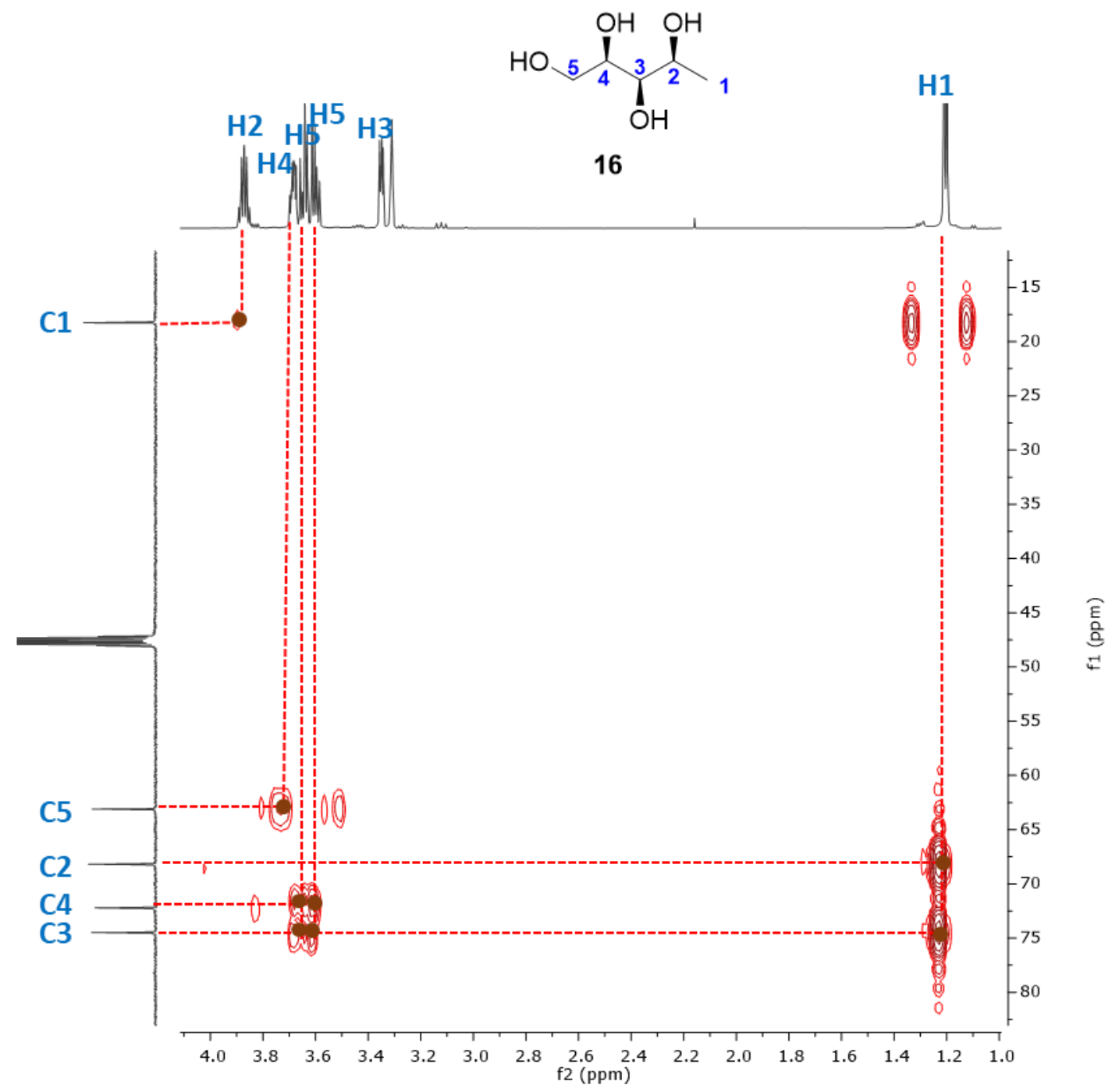


${ }^{1} \mathrm{H}$ NMR $\left(600 \mathrm{MHz}, \mathrm{CD}_{3} \mathrm{OD}\right)$

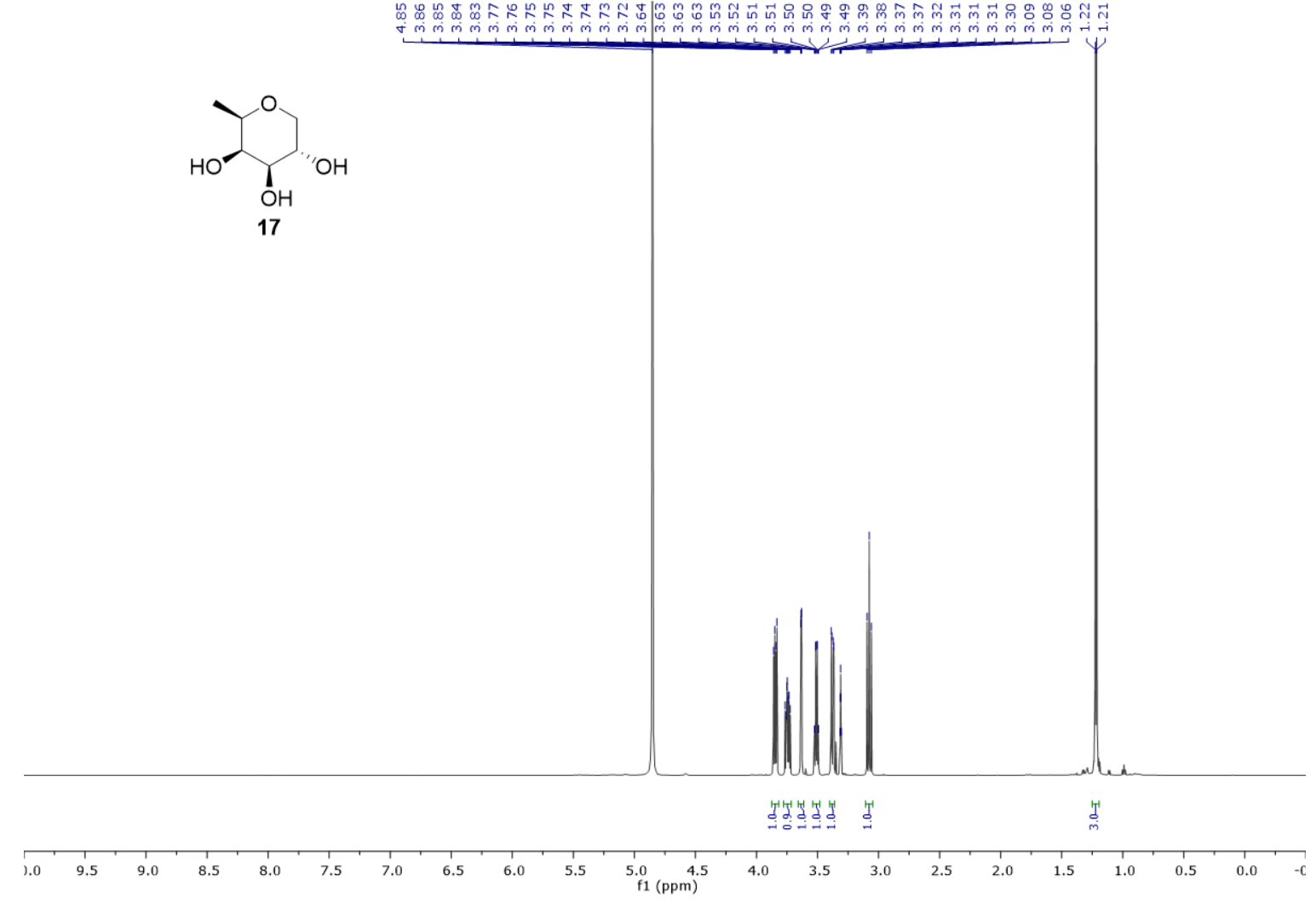

${ }^{13} \mathrm{C}$ NMR $\left(151 \mathrm{MHz}, \mathrm{CD}_{3} \mathrm{OD}\right)$
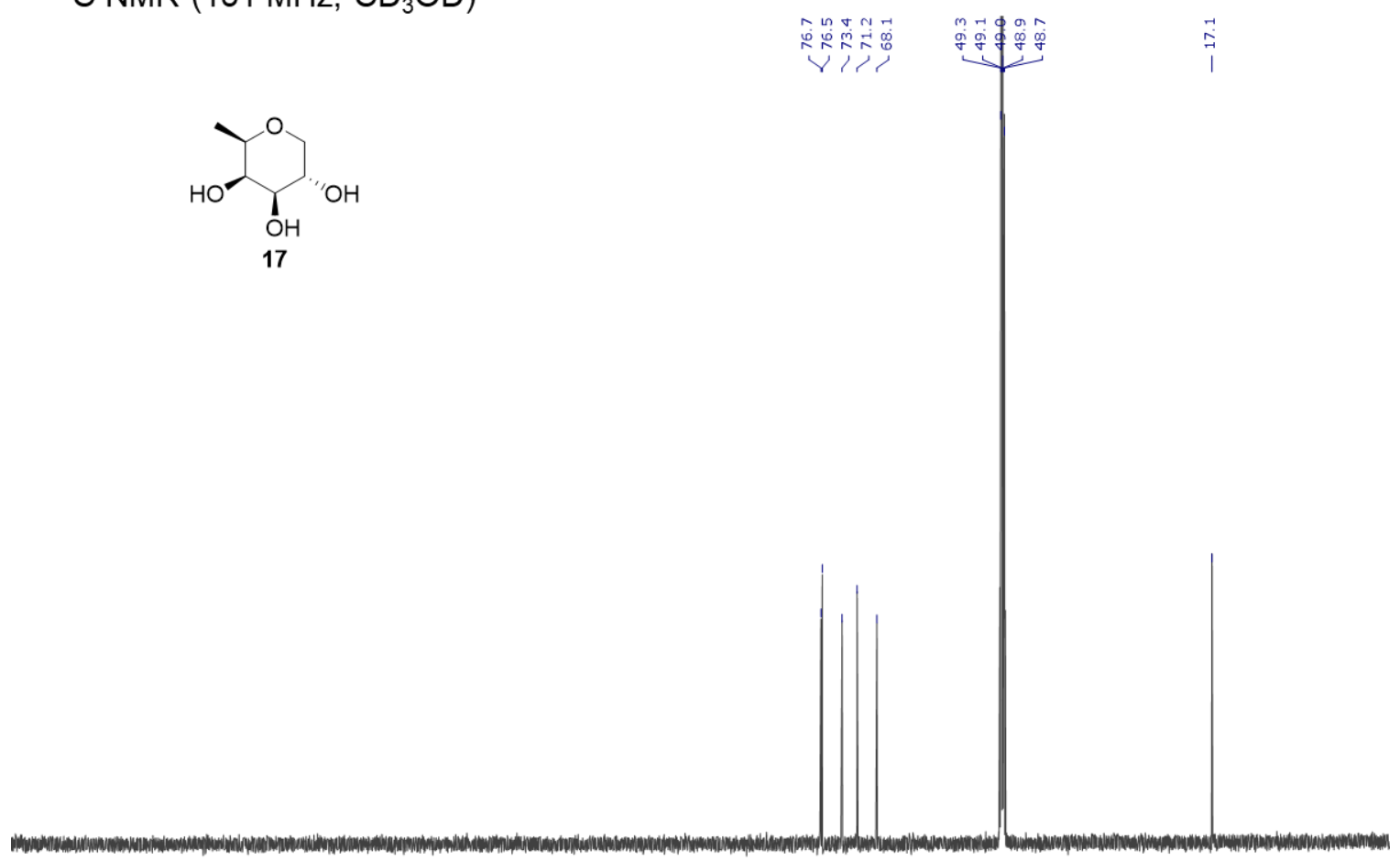

$\begin{array}{llllllllll}190 & 180 & 170 & 160 & 150 & 140 & 130 & 120 & 110 & \begin{array}{c}100 \\ \mathrm{f} 1(\mathrm{ppm})\end{array}\end{array}$ 
${ }^{1} \mathrm{H}$ NMR $\left(600 \mathrm{MHz}, \mathrm{CD}_{2} \mathrm{Cl}_{2}\right)$
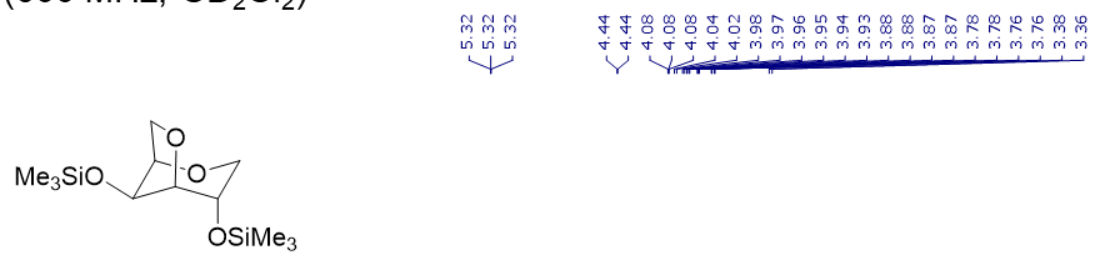

18

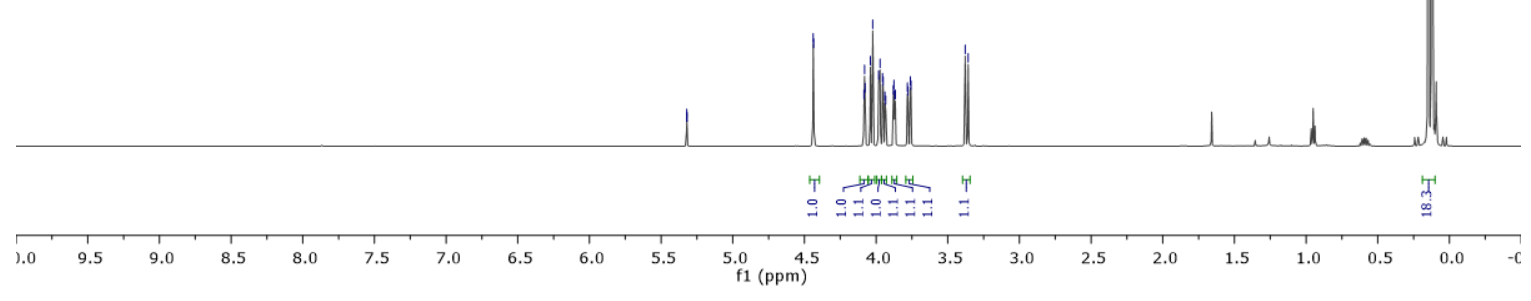

${ }^{13} \mathrm{C}$ NMR $\left(151 \mathrm{MHz}, \mathrm{CD}_{2} \mathrm{Cl}_{2}\right.$ )

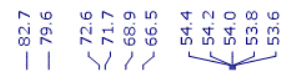

צִ

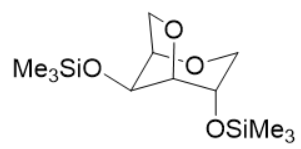

18

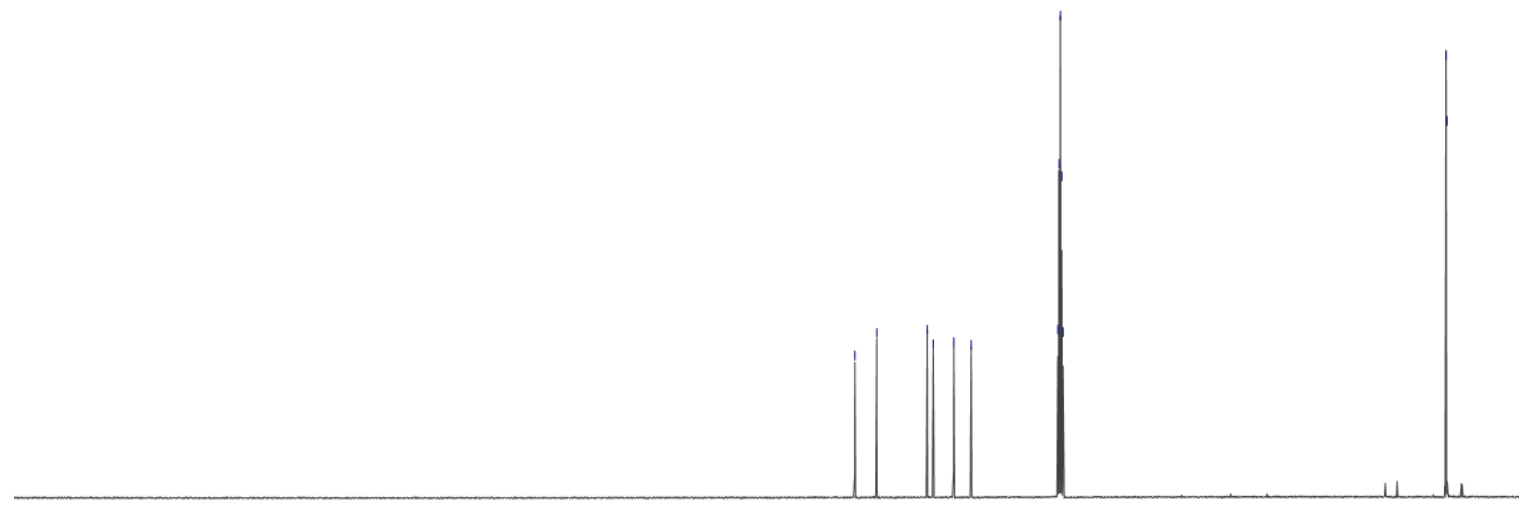




\section{Computational Details}

Calculations were carried out with the Gaussian 09 program package, revision E.01. Geometry optimizations and frequency analyses were performed using the M06-2X functional with the 6$31+\mathrm{G}(\mathrm{d}, \mathrm{p})$ basis set $(\mathrm{M} 06-2 \mathrm{X} / 6-31+\mathrm{G}(\mathrm{d}, \mathrm{p}))$. The conductor-like polarizable continuum (CPCM) solvation model was used with solvent parameters for dichloromethane.

Table of Summarized Energies

\begin{tabular}{|c|c|c|c|c|}
\hline Compound & $\begin{array}{c}\text { Absolute E } \\
\quad \text { (a. u.) }\end{array}$ & $\begin{array}{l}\text { \# imaginary } \\
\text { freq }\end{array}$ & $\begin{array}{l}\text { Zero Point E } \\
\quad \text { (a. u.) }\end{array}$ & G (a. u.) \\
\hline $\mathrm{Me}_{3} \mathrm{Si}-6-{ }^{4} C_{l}$ & -2245.592055 & 0 & -2245.634371 & -2245.708675 \\
\hline $\mathrm{Me}_{3} \mathrm{Si}-{ }^{-} C_{4}$ & -2245.582666 & 0 & -2245.625733 & -2245.703757 \\
\hline $\mathrm{Et}_{3} \mathrm{Si}-\mathbf{6}-{ }^{4} C_{1}$ & -2716.721296 & 0 & -2716.778434 & -2716.870935 \\
\hline $\mathrm{Et}_{3} \mathrm{Si}^{-6}-{ }^{I} C_{4}$ & -2716.709386 & 0 & -2716.766816 & -2716.862623 \\
\hline $\mathrm{Me}_{3} \mathrm{Si}-7-{ }^{4} C_{1}$ & -2245.586221 & 0 & -2245.628705 & -2245.702703 \\
\hline $\mathrm{Me}_{3} \mathrm{Si}-7-{ }^{I} C_{4}$ & -2245.586519 & 0 & -2245.629678 & -2245.70688 \\
\hline $\mathrm{Et}_{3} \mathrm{Si}_{-}{ }^{-}{ }^{4} C_{1}$ & -2716.714273 & 0 & -2716.771401 & -2716.863575 \\
\hline $\mathrm{Et}_{3} \mathrm{Si}_{-7-}{ }^{1} C_{4}$ & -2716.714371 & 0 & -2716.771918 & -2716.866782 \\
\hline $\mathrm{Me}_{3} \mathrm{Si}-8-{ }^{4} C_{l}$ & -2245.586761 & 0 & -2245.629455 & -2245.704226 \\
\hline $\mathrm{Me}_{3} \mathrm{Si}-8-{ }^{I} C_{4}$ & -2245.585383 & 0 & -2245.628131 & -2245.703487 \\
\hline $\mathrm{Et}_{3} \mathrm{Si-8}-{ }^{4} C_{1}$ & -2716.715929 & 0 & -2716.773199 & -2716.865607 \\
\hline $\mathrm{Et}_{3} \mathrm{Si}-8-{ }^{I} C_{4}$ & -2716.711796 & 0 & -2716.768943 & -2716.861458 \\
\hline
\end{tabular}

\section{Full Gaussian References}

Gaussian 09, Revision E.01, M. J. Frisch, G. W. Trucks, H. B. Schlegel, G. E. Scuseria, M. A. Robb, J. R. Cheeseman, G. Scalmani, V. Barone, G. A. Petersson, H. Nakatsuji, X. Li, M Caricato, A. Marenich, J. Bloino, B. G. Janeska, R. Gomperts, B. Mennucci, H. P. Hratchian, J. B. Ortiz, A. F. Izmaylov, J. L. Sonnenberg, D. Williams-Young, F. Ding, F. Lipparini, R. Egidi, J. Goings, B. Peng, A. Petrone, T. Henderson, D. Ranasinghe, B. G. Zakrzewski, J. Gao, N. Rega, G. Zheng, W. Liang, M. Hada, M. Ehara, K. Toyota, R. Fukuda, J. Hasegawa, M. Ishida, T. Nakajima, Y. Honda, O. Kitao, H. Nakai, T. Vreve, K. Throssel, J. A. Montgomery, Jr., J. E. Peralta, F. Ogliaro, M. Bearpark, J. J. Heyd, E. Brothers, K. N. Kudin, V. N. Staroverov, T. Keith, R. Kobayashi, J. Normand, K. Raghavachari, A. Rendell, J. C. Burant, S. S. Iyengar, J. Tomasi, M. Cossi, J. M. Millam, M. Klene, C. Adamo, R. Cammi, J. W. Ochterski, R. L. Martin, K. Morokuma, O. Farka, J. B. Foresman, and D. J. Fox, Gaussian, Inc., Wallingford CT, 2016. 
Optimized Coordinates for each structure

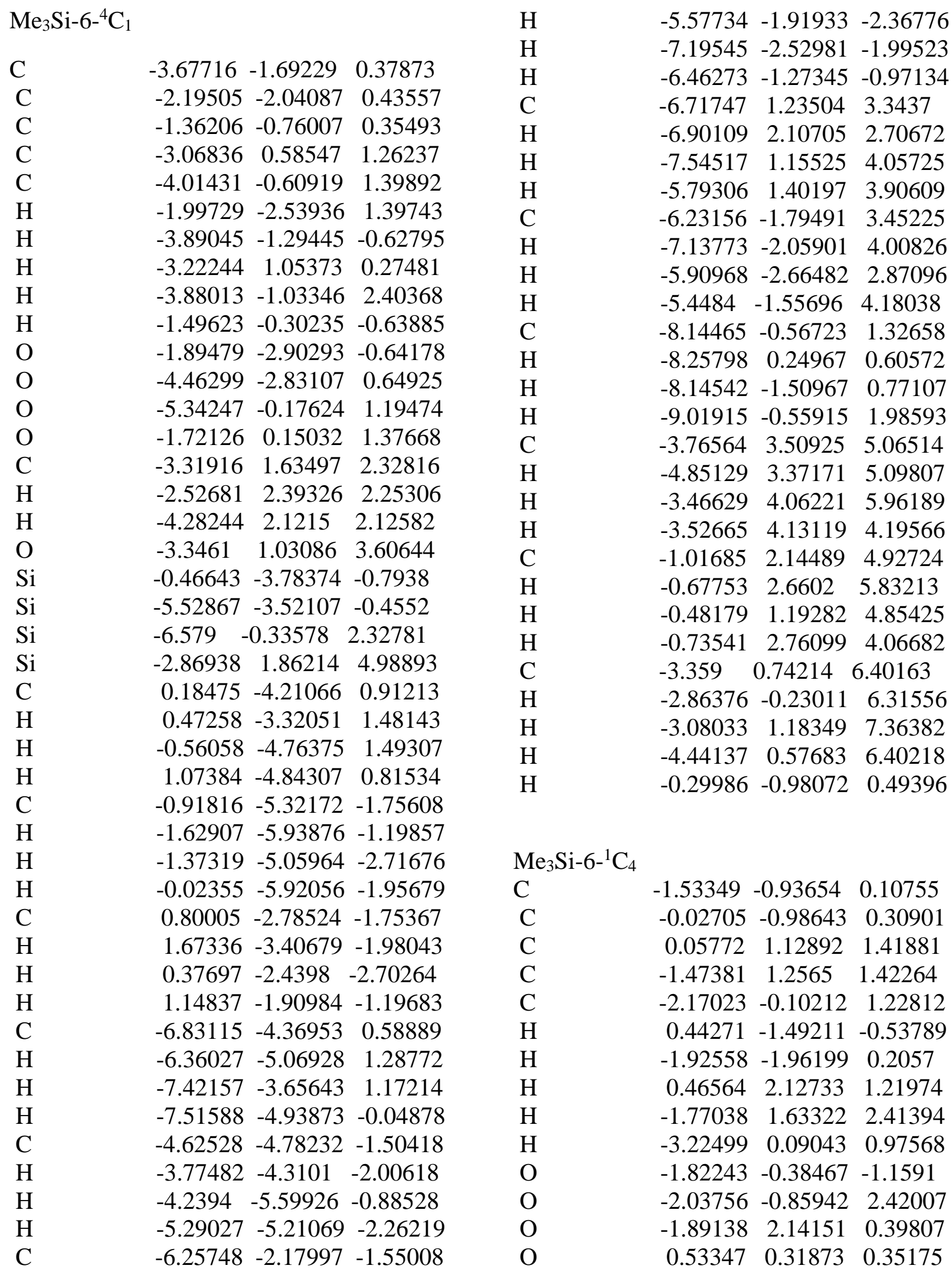




\begin{tabular}{|c|c|c|c|c|c|c|c|}
\hline $\mathrm{C}$ & 0.62546 & 0.6741 & 2.76794 & $\mathrm{H}$ & 2.99395 & 2.67331 & 2.45871 \\
\hline $\mathrm{H}$ & 1.71005 & 0.53808 & 2.64546 & $\mathrm{H}$ & 1.97502 & 4.11986 & 2.58523 \\
\hline $\mathrm{H}$ & 0.19223 & -0.2773 & 3.08733 & $\mathrm{C}$ & 2.60568 & 1.43396 & 5.51642 \\
\hline $\mathrm{O}$ & 0.31564 & 1.65847 & 3.74383 & $\mathrm{H}$ & 3.39537 & 2.00734 & 6.01343 \\
\hline $\mathrm{Si}$ & -2.95216 & -1.07509 & -2.19324 & $\mathrm{H}$ & 2.01298 & 0.93073 & 6.28691 \\
\hline $\mathrm{Si}$ & -3.30358 & -1.06128 & 3.51266 & $\mathrm{H}$ & 3.08753 & 0.66731 & 4.90059 \\
\hline $\mathrm{Si}$ & -2.29736 & 3.73745 & 0.73638 & $\mathrm{C}$ & 0.62593 & 3.81213 & 5.53673 \\
\hline $\mathrm{Si}$ & 1.52899 & 2.56588 & 4.47828 & $\mathrm{H}$ & 1.33725 & 4.42769 & 6.09691 \\
\hline $\mathrm{C}$ & -4.57484 & -1.32295 & -1.28222 & $\mathrm{H}$ & 0.01144 & 4.47764 & 4.92229 \\
\hline $\mathrm{H}$ & -4.45979 & -2.01694 & -0.44196 & $\mathrm{H}$ & -0.02726 & 3.30792 & 6.25584 \\
\hline $\mathrm{H}$ & -4.96283 & -0.37471 & -0.89513 & $\mathrm{H}$ & 0.20116 & -1.54351 & 1.2271 \\
\hline $\mathrm{H}$ & -5.32542 & -1.74623 & -1.95855 & & & & \\
\hline $\mathrm{C}$ & -2.30853 & -2.73403 & -2.79037 & $\mathrm{Et}_{3} \mathrm{Si}-6-{ }^{4} C_{1}$ & & & \\
\hline $\mathrm{H}$ & -3.02492 & -3.19966 & -3.47561 & $\mathrm{C}$ & -3.65497 & -1.80809 & 0.42714 \\
\hline $\mathrm{H}$ & -1.35788 & -2.61409 & -3.31965 & $\mathrm{C}$ & -2.19219 & -2.23443 & 0.49684 \\
\hline $\mathrm{H}$ & -2.14894 & -3.42444 & -1.95511 & $\mathrm{C}$ & -1.29858 & -0.99907 & 0.38049 \\
\hline $\mathrm{C}$ & -3.12783 & 0.14305 & -3.59788 & $\mathrm{C}$ & -2.932 & 0.43994 & 1.28186 \\
\hline $\mathrm{H}$ & -2.16504 & 0.30523 & -4.09224 & $\mathrm{C}$ & -3.93356 & -0.70858 & 1.4541 \\
\hline $\mathrm{H}$ & -3.83797 & -0.22579 & -4.34485 & $\mathrm{H}$ & -2.02329 & -2.71347 & 1.4741 \\
\hline $\mathrm{H}$ & -3.48975 & 1.10733 & -3.22754 & $\mathrm{H}$ & -3.83671 & -1.38953 & -0.57806 \\
\hline $\mathrm{C}$ & -3.94494 & 0.61699 & 4.04856 & $\mathrm{H}$ & -3.08036 & 0.89059 & 0.28502 \\
\hline $\mathrm{H}$ & -3.15687 & 1.21604 & 4.51759 & $\mathrm{H}$ & -3.79818 & -1.1296 & 2.46082 \\
\hline $\mathrm{H}$ & -4.34026 & 1.17529 & 3.19278 & $\mathrm{H}$ & -1.42249 & -0.55583 & -0.62133 \\
\hline $\mathrm{H}$ & -4.75929 & 0.49825 & 4.77137 & $\mathrm{O}$ & -1.93438 & -3.1431 & -0.54975 \\
\hline $\mathrm{C}$ & -2.5456 & -2.0039 & 4.93462 & $\mathrm{O}$ & -4.50016 & -2.90818 & 0.67572 \\
\hline $\mathrm{H}$ & -1.71025 & -1.44089 & 5.36357 & $\mathrm{O}$ & -5.24395 & -0.21454 & 1.27363 \\
\hline $\mathrm{H}$ & -3.28441 & -2.174 & 5.72408 & $\mathrm{O}$ & -1.60404 & -0.05333 & 1.38648 \\
\hline $\mathrm{H}$ & -2.17023 & -2.97667 & 4.60248 & $\mathrm{C}$ & -3.10939 & 1.53051 & 2.31911 \\
\hline $\mathrm{C}$ & -4.68433 & -2.02125 & 2.68256 & $\mathrm{H}$ & -2.28929 & 2.25316 & 2.19985 \\
\hline $\mathrm{H}$ & -5.13947 & -1.44327 & 1.87094 & $\mathrm{H}$ & -4.05775 & 2.04908 & 2.12087 \\
\hline $\mathrm{H}$ & -4.31316 & -2.96394 & 2.26728 & $\mathrm{O}$ & -3.11979 & 0.96896 & 3.61568 \\
\hline $\mathrm{H}$ & -5.47284 & -2.25328 & 3.40657 & $\mathrm{Si}$ & -0.49742 & -3.99135 & -0.8011 \\
\hline $\mathrm{C}$ & -3.81381 & 3.78385 & 1.84247 & $\mathrm{Si}$ & -5.61097 & -3.51486 & -0.4402 \\
\hline $\mathrm{H}$ & -4.61758 & 3.16069 & 1.4361 & $\mathrm{Si}$ & -6.49972 & -0.37444 & 2.39212 \\
\hline $\mathrm{H}$ & -3.58438 & 3.43561 & 2.85519 & $\mathrm{Si}$ & -2.85574 & 1.90404 & 4.99302 \\
\hline $\mathrm{H}$ & -4.18651 & 4.81097 & 1.92191 & $\mathrm{C}$ & 0.3231 & -4.36016 & 0.85806 \\
\hline $\mathrm{C}$ & -2.64263 & 4.50976 & -0.92858 & $\mathrm{H}$ & 0.20576 & -3.48776 & 1.51442 \\
\hline $\mathrm{H}$ & -2.92649 & 5.56088 & -0.81461 & $\mathrm{H}$ & -0.21837 & -5.18029 & 1.34769 \\
\hline $\mathrm{H}$ & -1.75598 & 4.46242 & -1.56825 & $\mathrm{C}$ & -1.04195 & -5.56654 & -1.67222 \\
\hline $\mathrm{H}$ & -3.46042 & 3.98893 & -1.43632 & $\mathrm{H}$ & -1.98235 & -5.87045 & -1.19549 \\
\hline $\mathrm{C}$ & -0.87025 & 4.57522 & 1.62373 & $\mathrm{H}$ & -1.29532 & -5.32767 & -2.71404 \\
\hline $\mathrm{H}$ & -0.59202 & 4.00501 & 2.51865 & $\mathrm{C}$ & 0.63658 & -2.94054 & -1.88781 \\
\hline $\mathrm{H}$ & 0.01188 & 4.6525 & 0.97923 & $\mathrm{H}$ & -0.01202 & -2.31667 & -2.51662 \\
\hline $\mathrm{H}$ & -1.15275 & 5.58471 & 1.94123 & $\mathrm{H}$ & 1.20274 & -2.24667 & -1.25177 \\
\hline $\mathrm{C}$ & 2.56991 & 3.40173 & 3.15844 & $\mathrm{C}$ & -6.89918 & -4.37176 & 0.63241 \\
\hline $\mathrm{H}$ & 3.40301 & 3.94195 & 3.62075 & $\mathrm{H}$ & -6.36376 & -4.8045 & 1.4886 \\
\hline
\end{tabular}




\begin{tabular}{|c|c|c|c|c|c|c|c|}
\hline $\mathrm{H}$ & -7.57414 & -3.61515 & 1.05394 & $\mathrm{C}$ & -7.70882 & -5.46523 & -0.08011 \\
\hline $\mathrm{C}$ & -4.74277 & -4.72979 & -1.58726 & $\mathrm{H}$ & -7.05281 & -6.25504 & -0.46127 \\
\hline $\mathrm{H}$ & -3.7557 & -4.29487 & -1.79068 & $\mathrm{H}$ & -8.43155 & -5.93342 & 0.59523 \\
\hline $\mathrm{H}$ & -4.5528 & -5.66222 & -1.03767 & $\mathrm{H}$ & -8.26735 & -5.06429 & -0.93254 \\
\hline $\mathrm{C}$ & -6.33659 & -2.08745 & -1.44029 & $\mathrm{C}$ & -9.3428 & 0.01119 & 2.03207 \\
\hline $\mathrm{H}$ & -5.66502 & -1.86328 & -2.28033 & $\mathrm{H}$ & -9.25886 & 1.04634 & 2.37916 \\
\hline $\mathrm{H}$ & -6.32972 & -1.19793 & $3-0.79794$ & $\mathrm{H}$ & -10.2045 & $\begin{array}{ll}7 & -0.0426\end{array}$ & 11.3596 \\
\hline $\mathrm{C}$ & -6.50229 & 1.13359 & 3.52485 & $\mathrm{H}$ & -9.56868 & -0.60936 & 2.90568 \\
\hline $\mathrm{H}$ & -6.84181 & 2.01378 & 2.96146 & $\mathrm{C}$ & -7.30263 & 0.98188 & 4.82664 \\
\hline $\mathrm{H}$ & -5.4489 & 1.30966 & 3.77368 & $\mathrm{H}$ & -7.22667 & 1.88079 & 5.44858 \\
\hline $\mathrm{C}$ & -6.25648 & -1.942 & 3.40987 & $\mathrm{H}$ & -8.36558 & 0.8004 & 4.63863 \\
\hline $\mathrm{H}$ & -5.76858 & -2.67977 & 2.76063 & $\mathrm{H}$ & -6.92741 & 0.14113 & 5.42069 \\
\hline $\mathrm{H}$ & -5.55076 & -1.73179 & 4.22619 & $\mathrm{C}$ & -7.55981 & -2.52458 & 3.98096 \\
\hline $\mathrm{C}$ & -8.05394 & -0.4392 & 1.33 & $\mathrm{H}$ & -7.37129 & -3.44328 & 4.54563 \\
\hline $\mathrm{H}$ & -7.86818 & 0.19677 & 0.45348 & $\mathrm{H}$ & -8.06243 & -1.82237 & 4.65462 \\
\hline $\mathrm{H}$ & -8.17031 & -1.45825 & 0.9385 & $\mathrm{H}$ & -8.26822 & -2.77195 & 3.18218 \\
\hline $\mathrm{C}$ & -3.69669 & 3.58081 & 4.7698 & $\mathrm{C}$ & -3.07015 & 1.18706 & 7.78045 \\
\hline $\mathrm{H}$ & -4.60395 & 3.43072 & 4.16907 & $\mathrm{H}$ & -3.55179 & 0.56424 & 8.54041 \\
\hline $\mathrm{H}$ & -3.04339 & 4.23511 & 4.17685 & $\mathrm{H}$ & -1.99484 & 0.98378 & 7.82097 \\
\hline $\mathrm{C}$ & -0.99579 & 2.11115 & 5.2125 & $\mathrm{H}$ & -3.21726 & 2.23236 & 8.07171 \\
\hline $\mathrm{H}$ & -0.58473 & 1.16606 & 5.59111 & $\mathrm{C}$ & -4.0742 & 4.26869 & 6.09214 \\
\hline $\mathrm{H}$ & -0.56548 & 2.24581 & 4.21059 & $\mathrm{H}$ & -4.55401 & 5.23653 & 5.91769 \\
\hline $\mathrm{C}$ & -3.63345 & 0.90294 & 6.38016 & $\mathrm{H}$ & -4.77299 & 3.65336 & 6.66923 \\
\hline $\mathrm{H}$ & -3.48656 & -0.15557 & 6.12753 & $\mathrm{H}$ & -3.19697 & 4.44683 & 6.72294 \\
\hline $\mathrm{H}$ & -4.719 & 1.07068 & 6.36421 & $\mathrm{C}$ & -0.57284 & 3.2799 & 6.11513 \\
\hline $\mathrm{H}$ & -0.24564 & -1.26677 & 0.51194 & $\mathrm{H}$ & 0.51618 & 3.35295 & 6.1949 \\
\hline $\mathrm{C}$ & 1.8183 & -4.70028 & 0.74395 & $\mathrm{H}$ & -0.93856 & 4.23384 & 5.72067 \\
\hline $\mathrm{H}$ & 2.25502 & -4.91428 & 1.72413 & $\mathrm{H}$ & -0.96913 & 3.17176 & 7.13023 \\
\hline $\mathrm{H}$ & 1.99044 & -5.57612 & 0.10986 & & & & \\
\hline $\mathrm{H}$ & 2.37924 & -3.86619 & 0.30897 & $\mathrm{Et}_{3} \mathrm{Si}^{-6}-{ }^{1} C_{4}$ & & & \\
\hline $\mathrm{C}$ & 1.60155 & -3.74224 & -2.77492 & $\mathrm{C}$ & -1.54109 & -0.96046 & 0.03313 \\
\hline $\mathrm{H}$ & 1.05404 & -4.40842 & -3.45029 & $\mathrm{C}$ & -0.02678 & -1.05754 & 0.15147 \\
\hline $\mathrm{H}$ & 2.2211 & -3.08379 & -3.39144 & $\mathrm{C}$ & 0.17431 & 1.04518 & 1.28342 \\
\hline $\mathrm{H}$ & 2.27683 & -4.3641 & -2.1777 & $\mathrm{C}$ & -1.35077 & 1.21875 & 1.35318 \\
\hline $\mathrm{C}$ & -0.04136 & -6.73106 & -1.61802 & $\mathrm{C}$ & -2.08987 & -0.1179 & 1.19205 \\
\hline $\mathrm{H}$ & -0.43351 & -7.61569 & -2.12915 & $\mathrm{H}$ & 0.38086 & -1.55525 & -0.73165 \\
\hline $\mathrm{H}$ & 0.91241 & -6.47572 & -2.09055 & $\mathrm{H}$ & -1.95642 & -1.97554 & 0.14765 \\
\hline $\mathrm{H}$ & 0.17117 & -7.01788 & -0.5827 & $\mathrm{H}$ & 0.59112 & 2.03426 & 1.06014 \\
\hline $\mathrm{C}$ & -5.46997 & -5.02551 & -2.90746 & $\mathrm{H}$ & -1.59261 & 1.60004 & 2.35632 \\
\hline $\mathrm{H}$ & -5.61999 & -4.10853 & -3.48776 & $\mathrm{H}$ & -3.15291 & 0.09885 & 1.00236 \\
\hline $\mathrm{H}$ & -4.89648 & -5.71737 & -3.53313 & $\mathrm{O}$ & -1.89148 & -0.38917 & -1.20946 \\
\hline $\mathrm{H}$ & -6.45607 & -5.47293 & -2.74393 & $\mathrm{O}$ & -1.90105 & -0.87411 & 2.37677 \\
\hline $\mathrm{C}$ & -7.75664 & -2.34745 & -1.96704 & $\mathrm{O}$ & -1.7735 & 2.12227 & 0.34709 \\
\hline $\mathrm{H}$ & -8.14119 & -1.48595 & -2.52251 & $\mathrm{O}$ & 0.58176 & 0.22667 & 0.19423 \\
\hline $\mathrm{H}$ & -7.78921 & -3.21244 & -2.63782 & $\mathrm{C}$ & 0.79693 & 0.56971 & 2.60203 \\
\hline $\mathrm{H}$ & -8.45597 & -2.5469 & -1.14746 & $\mathrm{H}$ & 1.88869 & 0.54959 & 2.4707 \\
\hline
\end{tabular}




\begin{tabular}{|c|c|c|c|c|c|c|c|}
\hline $\mathrm{H}$ & 0.45998 & -0.43806 & $5 \quad 2.85902$ & $\mathrm{H}$ & -3.92105 & -4.07008 & -2.69389 \\
\hline $\mathrm{O}$ & 0.4025 & 1.45294 & 3.6392 & $\mathrm{C}$ & -3.7951 & -0.43345 & -4.92348 \\
\hline $\mathrm{Si}$ & -3.00261 & -1.12277 & -2.23887 & $\mathrm{H}$ & -3.93495 & 0.35041 & -5.67399 \\
\hline $\mathrm{Si}$ & -3.1423 & -1.10558 & 3.49505 & $\mathrm{H}$ & -3.08139 & -1.15638 & -5.33242 \\
\hline $\mathrm{Si}$ & -2.17036 & 3.71628 & 0.71625 & $\mathrm{H}$ & -4.75361 & -0.94986 & -4.80665 \\
\hline $\mathrm{Si}$ & 1.48669 & 2.43872 & 4.46797 & $\mathrm{C}$ & -5.84085 & -1.63294 & -2.14643 \\
\hline $\mathrm{C}$ & -4.58097 & -1.50309 & -1.27432 & $\mathrm{H}$ & -6.7249 & -1.85471 & -1.5408 \\
\hline $\mathrm{H}$ & -4.43784 & $4-2.41755$ & $5-0.68263$ & $\mathrm{H}$ & -6.03923 & -0.70351 & -2.69035 \\
\hline $\mathrm{H}$ & -4.72797 & $7 \quad-0.69149$ & $9-0.54904$ & $\mathrm{H}$ & -5.74235 & -2.43215 & -2.888 \\
\hline $\mathrm{C}$ & -2.22651 & -2.70871 & $1-2.90311$ & $\mathrm{C}$ & -5.40581 & -2.88902 & 3.62137 \\
\hline $\mathrm{H}$ & -1.48706 & $5-2.43337$ & $\begin{array}{ll}7 & -3.66697\end{array}$ & $\mathrm{H}$ & -5.90598 & -2.24035 & 4.34807 \\
\hline $\mathrm{H}$ & -1.65336 & $5-3.16158$ & $8-2.08205$ & $\mathrm{H}$ & -6.18364 & -3.44079 & 3.08487 \\
\hline $\mathrm{C}$ & -3.29316 & 0.14661 & -3.59221 & $\mathrm{H}$ & -4.81454 & -3.61792 & 4.18555 \\
\hline $\mathrm{H}$ & -2.33871 & 0.66496 & $5-3.75019$ & $\mathrm{C}$ & -5.15453 & 0.58703 & 4.7086 \\
\hline $\mathrm{H}$ & -3.9928 & 0.9053 & -3.21772 & $\mathrm{H}$ & -5.45788 & 1.59952 & 4.9928 \\
\hline $\mathrm{C}$ & -3.76985 & 0.5838 & 4.04303 & $\mathrm{H}$ & -5.91754 & 0.19511 & 4.02789 \\
\hline $\mathrm{H}$ & -3.0211 & 1.04174 & 4.70418 & $\mathrm{H}$ & -5.17425 & -0.02857 & 5.61371 \\
\hline $\mathrm{H}$ & -3.80625 & $5 \quad 1.21823$ & 33.14731 & $\mathrm{C}$ & -3.02987 & -1.94161 & 6.24966 \\
\hline $\mathrm{C}$ & -2.32297 & $7-2.05787$ & $7 \quad 4.8904$ & $\mathrm{H}$ & -4.05809 & -2.31577 & 6.20642 \\
\hline $\mathrm{H}$ & -1.29777 & $7-1.67311$ & 14.97203 & $\mathrm{H}$ & -2.50593 & -2.51198 & 7.02248 \\
\hline $\mathrm{H}$ & -2.23182 & $2-3.11143$ & $\begin{array}{ll}3 & 4.59636\end{array}$ & $\mathrm{H}$ & -3.07342 & -0.89941 & 6.58278 \\
\hline $\mathrm{C}$ & -4.51824 & -2.08559 & 2.65658 & $\mathrm{C}$ & -4.56293 & 5.02472 & 1.66115 \\
\hline $\mathrm{H}$ & -5.13594 & $4-1.40371$ & 12.05622 & $\mathrm{H}$ & -4.89031 & 5.19395 & 0.63003 \\
\hline $\mathrm{H}$ & -4.03905 & $5-2.76902$ & $2 \quad 1.94228$ & $\mathrm{H}$ & -5.45855 & 4.98183 & 2.28841 \\
\hline $\mathrm{C}$ & -3.73496 & 53.73388 & 1.77359 & $\mathrm{H}$ & -3.98786 & 5.90362 & 1.97037 \\
\hline $\mathrm{H}$ & -4.34948 & 2.87759 & 1.46285 & $\mathrm{C}$ & -2.28399 & 6.04584 & -0.98647 \\
\hline $\mathrm{H}$ & -3.46491 & 3.55294 & 42.82336 & $\mathrm{H}$ & -2.45257 & 6.4481 & -1.99 \\
\hline $\mathrm{C}$ & -2.44412 & 4.5181 & -0.96084 & $\mathrm{H}$ & -2.99073 & 6.5405 & -0.31209 \\
\hline $\mathrm{H}$ & -1.72445 & 54.05909 & -1.65049 & $\mathrm{H}$ & -1.2753 & 6.34114 & -0.67914 \\
\hline $\mathrm{H}$ & -3.43946 & $5 \quad 4.2338$ & -1.32673 & $\mathrm{C}$ & -1.09613 & 5.78537 & 2.43352 \\
\hline $\mathrm{C}$ & -0.74338 & 34.49554 & 1.67637 & $\mathrm{H}$ & -1.86698 & 5.59874 & 3.18925 \\
\hline $\mathrm{H}$ & -0.39229 & 7 3.74147 & $7 \quad 2.39664$ & $\mathrm{H}$ & -0.22312 & 6.19749 & 2.95081 \\
\hline $\mathrm{H}$ & 0.09515 & 4.67236 & 0.98834 & $\mathrm{H}$ & -1.47467 & 6.56346 & 1.76285 \\
\hline $\mathrm{C}$ & 2.62607 & 3.29954 & 3.23154 & $\mathrm{C}$ & 0.99415 & 4.28486 & 6.63616 \\
\hline $\mathrm{H}$ & 3.47426 & 2.64202 & 2.99934 & $\mathrm{H}$ & 0.29151 & 4.9648 & 7.12736 \\
\hline $\mathrm{H}$ & 2.07494 & 3.4317 & 2.29131 & $\mathrm{H}$ & 1.27873 & 3.52103 & 7.36737 \\
\hline $\mathrm{C}$ & 2.4958 & 1.36809 & 5.64525 & $\mathrm{H}$ & 1.89387 & 4.86088 & 6.39592 \\
\hline $\mathrm{H}$ & 1.8555 & 1.07626 & 6.48814 & $\mathrm{C}$ & 3.13826 & 4.66673 & 3.71435 \\
\hline $\mathrm{H}$ & 2.73477 & 0.43773 & 5.11168 & $\mathrm{H}$ & 3.79364 & 5.13409 & 2.97324 \\
\hline $\mathrm{C}$ & 0.37522 & 3.64846 & 5.38248 & $\mathrm{H}$ & 2.30638 & 5.35491 & 3.90024 \\
\hline $\mathrm{H}$ & 0.05034 & 4.42992 & 4.68289 & $\mathrm{H}$ & 3.70634 & 4.58052 & 4.64679 \\
\hline $\mathrm{H}$ & -0.53176 & $5 \quad 3.09339$ & 5.65683 & $\mathrm{C}$ & 3.79319 & 2.01322 & 6.15674 \\
\hline $\mathrm{H}$ & 0.23088 & -1.64657 & $7 \quad 1.04076$ & $\mathrm{H}$ & 4.33956 & 1.34129 & 6.82566 \\
\hline $\mathrm{C}$ & -3.21475 & -3.74249 & -3.46401 & $\mathrm{H}$ & 4.46202 & 2.26547 & 5.32706 \\
\hline $\mathrm{H}$ & -3.80102 & $2-3.33447$ & $7 \quad-4.29403$ & $\mathrm{H}$ & 3.5955 & 2.93685 & 6.71052 \\
\hline $\mathrm{H}$ & -2.69741 & -4.6326 & -3.83497 & & & & \\
\hline
\end{tabular}




\begin{tabular}{|c|c|c|c|c|c|c|}
\hline $\mathrm{Me}_{3} \mathrm{Si}-7-{ }^{4} C_{1}$ & & & $\mathrm{H}$ & -0.42746 & $6-2.24584$ & -2.9185 \\
\hline $\mathrm{C}$ & -0.47909 & $0.16858 \quad 0.0207$ & $\mathrm{C}$ & -1.60767 & $7-0.41177$ & 5.03187 \\
\hline $\mathrm{C}$ & 1.01543 & $-0.05883 \quad 0.19344$ & $\mathrm{H}$ & -0.85643 & $3-1.20769$ & 5.02118 \\
\hline $\mathrm{C}$ & 1.72362 & 0.3971 & $\mathrm{H}$ & -1.1356 & 0.50011 & 5.41032 \\
\hline $\mathrm{C}$ & -0.19826 & $2.29945 \quad 1.29235$ & $\mathrm{H}$ & -2.40543 & $3-0.70226$ & 5.72302 \\
\hline $\mathrm{C}$ & -1.02088 & 1.009121 .18316 & $\mathrm{C}$ & -3.14481 & -1.62009 & 2.63107 \\
\hline $\mathrm{H}$ & 1.16034 & $-0.68843 \quad 1.08411$ & $\mathrm{H}$ & -3.54625 & $5-1.40177$ & 7.63568 \\
\hline $\mathrm{H}$ & -0.61206 & $0.74771-0.91018$ & $\mathrm{H}$ & -2.44878 & $8-2.45934$ & 2.53308 \\
\hline $\mathrm{H}$ & -0.32632 & 2.840290 .33619 & $\mathrm{H}$ & -3.97559 & $9-1.92701$ & 3.27583 \\
\hline $\mathrm{H}$ & -2.06057 & $1.28802 \quad 0.94543$ & $\mathrm{C}$ & -3.50323 & 31.32747 & 3.32476 \\
\hline $\mathrm{H}$ & 1.64713 & $1.86352-0.53574$ & $\mathrm{H}$ & -4.30082 & 21.11576 & 4.04621 \\
\hline $\mathrm{C}$ & -0.63982 & $3.27039 \quad 2.37747$ & $\mathrm{H}$ & -3.01087 & $7 \quad 2.25553$ & 3.62799 \\
\hline $\mathrm{H}$ & -0.07825 & $4.20298 \quad 2.23182$ & $\mathrm{H}$ & -3.97478 & $8 \quad 1.47954$ & 2.34802 \\
\hline $\mathrm{H}$ & -1.70633 & $3.49308 \quad 2.21787$ & $\mathrm{C}$ & 0.74804 & 2.87856 & 6.25859 \\
\hline $\mathrm{O}$ & 1.54302 & $-0.66274-0.97112$ & $\mathrm{H}$ & 0.78752 & 3.43863 & 7.19886 \\
\hline $\mathrm{O}$ & -1.14934 & $-1.06894-0.04726$ & $\mathrm{H}$ & 0.42878 & 1.85773 & 6.48881 \\
\hline $\mathrm{O}$ & -0.94822 & $0.25832 \quad 2.37051$ & $\mathrm{H}$ & 1.76007 & 72.83404 & 5.84446 \\
\hline $\mathrm{O}$ & -0.4191 & $2.76235 \quad 3.67398$ & $\mathrm{C}$ & -2.14914 & 3.80765 & 5.77395 \\
\hline $\mathrm{O}$ & 1.17654 & 2.010621 .47989 & $\mathrm{H}$ & -2.14475 & $5 \quad 4.41311$ & 6.687 \\
\hline $\mathrm{Si}$ & 2.32212 & $-2.15449-0.91365$ & $\mathrm{H}$ & -2.84974 & $4 \quad 4.26808$ & 5.06904 \\
\hline $\mathrm{Si}$ & -2.16517 & $-1.44858-1.33121$ & $\mathrm{H}$ & -2.52611 & 12.81142 & 26.02903 \\
\hline $\mathrm{Si}$ & -2.2848 & $-0.10142 \quad 3.31816$ & $\mathrm{C}$ & 0.19086 & 5.44176 & 4.64243 \\
\hline $\mathrm{Si}$ & -0.41694 & $3.7152 \quad 5.05759$ & $\mathrm{H}$ & 0.29196 & 6.02125 & 5.56644 \\
\hline $\mathrm{C}$ & 3.75727 & $-2.04248 \quad 0.29119$ & $\mathrm{H}$ & 1.17063 & 3.41459 & 4.15424 \\
\hline $\mathrm{H}$ & 4.4601 & $-1.25996-0.0127$ & $\mathrm{H}$ & -0.50549 & $9 \quad 5.97723$ & 3.98906 \\
\hline $\mathrm{H}$ & 3.41588 & -1.819351 .30772 & $\mathrm{H}$ & 2.78113 & 31.11703 & 0.61882 \\
\hline $\mathrm{H}$ & 4.30023 & $\begin{array}{ll}-2.99305 & 0.32471\end{array}$ & & & & \\
\hline $\mathrm{C}$ & 2.92389 & $-2.44639-2.65879$ & $\mathrm{Me}_{3} \mathrm{Si}-7-{ }^{1} C_{4}$ & & & \\
\hline $\mathrm{H}$ & 3.43238 & $-3.41312-2.73257$ & $\mathrm{C}$ & -0.2584 & -0.21862 & -0.22452 \\
\hline $\mathrm{H}$ & 2.08838 & $-2.44736-3.36581$ & $\mathrm{C}$ & 1.26942 & -0.10295 & -0.26299 \\
\hline $\mathrm{H}$ & 3.62875 & $-1.66634-2.96254$ & $\mathrm{C}$ & 1.68667 & 1.3572 & -0.06344 \\
\hline $\mathrm{C}$ & 1.12926 & $-3.49164-0.36371$ & $\mathrm{C}$ & 1.01752 & 1.90791 & 1.18676 \\
\hline $\mathrm{H}$ & 0.62244 & $-3.20936 \quad 0.56474$ & $\mathrm{C}$ & -0.89166 & $5 \quad 0.4789$ & 0.99371 \\
\hline $\mathrm{H}$ & 0.36066 & $-3.66322-1.12392$ & $\mathrm{H}$ & 2.77711 & 1.38357 & 0.08656 \\
\hline $\mathrm{H}$ & 1.66231 & $-4.43399-0.19757$ & $\mathrm{H}$ & 1.60632 & -0.44276 & $5-1.25393$ \\
\hline $\mathrm{C}$ & -3.52553 & $-0.15592-1.42857$ & $\mathrm{H}$ & -0.63203 & 30.29503 & $3-1.12452$ \\
\hline $\mathrm{H}$ & -4.08972 & $-0.10622-0.49078$ & $\mathrm{H}$ & 1.24371 & 2.97135 & 1.29363 \\
\hline $\mathrm{H}$ & -3.11627 & $0.84014-1.63156$ & $\mathrm{H}$ & -1.9651 & 0.59326 & 0.80843 \\
\hline $\mathrm{H}$ & -4.22738 & $-0.39657-2.23416$ & $\mathrm{C}$ & -0.77556 & $5-0.28373$ & $3 \quad 2.32085$ \\
\hline $\mathrm{C}$ & -2.85906 & $-3.14093-0.94567$ & $\mathrm{H}$ & -0.92719 & $9 \quad 0.45732$ & 23.12129 \\
\hline $\mathrm{H}$ & -2.05421 & $-3.86534-0.78687$ & $\mathrm{H}$ & 0.21288 & -0.7372 & 2.44844 \\
\hline $\mathrm{H}$ & -3.48097 & $-3.12 \quad-0.04602$ & $\mathrm{O}$ & 1.29044 & 2.11802 & -1.1901 \\
\hline $\mathrm{H}$ & -3.47526 & $-3.49436-1.77899$ & $\mathrm{O}$ & 1.89191 & -0.85713 & 30.7536 \\
\hline $\mathrm{C}$ & -1.19255 & $-1.46319-2.93507$ & $\mathrm{O}$ & -0.5761 & -1.58982 & -0.2399 \\
\hline $\mathrm{H}$ & -1.85897 & $-1.6528-3.78346$ & $\mathrm{O}$ & -1.77126 & $6-1.29345$ & $5 \quad 2.3591$ \\
\hline $\mathrm{H}$ & -0.68736 & $-0.50678-3.104$ & $\mathrm{O}$ & -0.39718 & $8 \quad 1.81064$ & $4 \quad 1.1019$ \\
\hline
\end{tabular}




\begin{tabular}{|c|c|c|c|c|c|c|}
\hline $\mathrm{Si}$ & 2.41601 & $2.59454-2.3501$ & $\mathrm{H}$ & -0.55897 & -1.1795 & 5.27897 \\
\hline $\mathrm{Si}$ & 2.13162 & -2.527720 .7633 & $\mathrm{H}$ & -1.63036 & -2.35657 & 6.05171 \\
\hline $\mathrm{Si}$ & -1.94381 & $-2.25573-0.95345$ & $\mathrm{C}$ & -3.43496 & -3.31689 & 3.40218 \\
\hline $\mathrm{Si}$ & -2.48796 & -1.756053 .80484 & $\mathrm{H}$ & -4.05184 & -3.62518 & 4.25254 \\
\hline $\mathrm{C}$ & 3.34818 & $1.08839-2.9725$ & $\mathrm{H}$ & -4.09415 & -3.16147 & 2.5416 \\
\hline $\mathrm{H}$ & 3.85557 & $0.56348-2.15496$ & $\mathrm{H}$ & -2.7482 & -4.13635 & 3.16621 \\
\hline $\mathrm{H}$ & 2.673 & $0.38152-3.46588$ & $\mathrm{H}$ & 1.39602 & 1.37164 & 2.06776 \\
\hline $\mathrm{H}$ & 4.11227 & $1.39002-3.6969$ & & & & \\
\hline $\mathrm{C}$ & 3.6139 & $3.81118-1.57377$ & $\mathrm{Et}_{3} \mathrm{Si}-7-{ }^{4} C_{1}$ & & & \\
\hline $\mathrm{H}$ & 4.34777 & $4.14935-2.31295$ & $\mathrm{C}$ & -0.39239 & 0.25814 & 0.09117 \\
\hline $\mathrm{H}$ & 3.08175 & $4.68952-1.195$ & $\mathrm{C}$ & 1.09923 & -0.01818 & 0.2358 \\
\hline $\mathrm{H}$ & 4.16393 & $3.35981-0.74139$ & $\mathrm{C}$ & 1.84829 & 1.3021 & 0.42418 \\
\hline $\mathrm{C}$ & 1.40845 & $3.39199 \quad-3.70476$ & $\mathrm{C}$ & -0.02706 & 2.36807 & 1.36144 \\
\hline $\mathrm{H}$ & 0.84234 & $4.24405-3.31621$ & $\mathrm{C}$ & -0.88428 & 1.09863 & 1.27627 \\
\hline $\mathrm{H}$ & 2.05916 & $3.75289-4.50763$ & $\mathrm{H}$ & 1.24534 & -0.65035 & 1.12465 \\
\hline $\mathrm{H}$ & 0.70096 & $2.67629-4.1346$ & $\mathrm{H}$ & -0.5252 & 0.85481 & -0.82882 \\
\hline $\mathrm{C}$ & 0.92149 & $-3.35981 \quad 1.92775$ & $\mathrm{H}$ & -0.16227 & 2.90481 & 0.404 \\
\hline $\mathrm{H}$ & 0.96758 & $-4.44897 \quad 1.81175$ & $\mathrm{H}$ & -1.92244 & 1.40522 & 1.07128 \\
\hline $\mathrm{H}$ & 1.18246 & -3.123592 .96598 & $\mathrm{H}$ & 1.76637 & 1.88785 & -0.50625 \\
\hline $\mathrm{H}$ & -0.1016 & $-3.02143 \quad 1.73795$ & $\mathrm{C}$ & -0.42202 & 3.35835 & 2.44489 \\
\hline $\mathrm{C}$ & 3.86409 & $-2.73618 \quad 1.45789$ & $\mathrm{H}$ & 0.16872 & 4.27195 & 2.28772 \\
\hline $\mathrm{H}$ & 4.10339 & $-3.79699 \quad 1.59031$ & $\mathrm{H}$ & -1.48196 & 3.61769 & 2.29163 \\
\hline $\mathrm{H}$ & 4.61265 & -2.298150 .78963 & $\mathrm{O}$ & 1.58289 & -0.63883 & -0.93798 \\
\hline $\mathrm{H}$ & 3.9504 & $-2.24737 \quad 2.43408$ & $\mathrm{O}$ & -1.10261 & -0.95505 & 0.01767 \\
\hline $\mathrm{C}$ & 2.08701 & $-3.22071-0.97919$ & $\mathrm{O}$ & -0.79509 & 0.34071 & 2.45858 \\
\hline $\mathrm{H}$ & 2.38331 & $-4.27541-0.95632$ & $\mathrm{O}$ & -0.2047 & 2.85131 & 3.74162 \\
\hline $\mathrm{H}$ & 1.09053 & $-3.14821-1.4212$ & $\mathrm{O}$ & 1.34381 & 2.04496 & 1.5192 \\
\hline $\mathrm{H}$ & 2.7949 & $-2.69097-1.62693$ & $\mathrm{Si}$ & 2.24445 & -2.19003 & -0.93869 \\
\hline $\mathrm{C}$ & -2.0056 & $-4.0015 \quad-0.29231$ & $\mathrm{Si}$ & -2.11527 & -1.3507 & -1.26725 \\
\hline $\mathrm{H}$ & -2.17904 & $\begin{array}{ll}-3.98572 & 0.78819\end{array}$ & $\mathrm{Si}$ & -2.11112 & -0.12584 & 3.39524 \\
\hline $\mathrm{H}$ & -2.80962 & $-4.57382-0.76605$ & $\mathrm{Si}$ & -0.40627 & 3.80949 & 5.1102 \\
\hline $\mathrm{H}$ & -1.05786 & $-4.5183 \quad-0.47854$ & $\mathrm{C}$ & 3.76856 & -2.1844 & 0.17604 \\
\hline $\mathrm{C}$ & -3.49395 & $-1.29242-0.52414$ & $\mathrm{H}$ & 4.23051 & -1.19215 & 0.08724 \\
\hline $\mathrm{H}$ & -4.36698 & $-1.7905-0.96063$ & $\mathrm{H}$ & 3.45575 & -2.28305 & 1.22426 \\
\hline $\mathrm{H}$ & -3.62248 & $-1.23779 \quad 0.56099$ & $\mathrm{C}$ & 2.70033 & -2.48267 & -2.73933 \\
\hline $\mathrm{H}$ & -3.46005 & $-0.27308-0.92492$ & $\mathrm{H}$ & 1.94165 & -1.97304 & -3.34725 \\
\hline $\mathrm{C}$ & -1.7018 & $-2.23498-2.81646$ & $\mathrm{H}$ & 3.65131 & -1.97659 & -2.95046 \\
\hline $\mathrm{H}$ & -1.54453 & $-1.21346-3.18012$ & $\mathrm{C}$ & 0.975 & -3.43662 & -0.31324 \\
\hline $\mathrm{H}$ & -0.83572 & $-2.83701-3.10995$ & $\mathrm{H}$ & 0.39911 & -2.94762 & 0.48291 \\
\hline $\mathrm{H}$ & -2.58414 & $-2.63784-3.32526$ & $\mathrm{H}$ & 0.25187 & -3.63096 & -1.11733 \\
\hline $\mathrm{C}$ & -3.63971 & $-0.39065 \quad 4.38079$ & $\mathrm{C}$ & -3.46891 & -0.03876 & -1.37457 \\
\hline $\mathrm{H}$ & -4.13282 & $-0.67099 \quad 5.3176$ & $\mathrm{H}$ & -3.71785 & 0.23835 & -0.34022 \\
\hline $\mathrm{H}$ & -3.09644 & $0.54395 \quad 4.55585$ & $\mathrm{H}$ & -3.07005 & 0.86933 & -1.84687 \\
\hline $\mathrm{H}$ & -4.41439 & $-0.19897 \quad 3.6311$ & $\mathrm{C}$ & -2.84276 & -3.0235 & -0.80383 \\
\hline $\mathrm{C}$ & -1.1682 & $-2.07201 \quad 5.10041$ & $\mathrm{H}$ & -2.08716 & -3.56133 & -0.21635 \\
\hline $\mathrm{H}$ & -0.50358 & $-2.8838 \quad 4.78847$ & $\mathrm{H}$ & -3.69495 & -2.86352 & -0.12933 \\
\hline
\end{tabular}




\begin{tabular}{|c|c|c|c|c|c|c|}
\hline $\mathrm{C}$ & $-1.10133-1.4206$ & -2.85653 & $\mathrm{C}$ & -4.10408 & -2.23457 & 3.15186 \\
\hline $\mathrm{H}$ & $-0.56144-2.37721$ & -2.8826 & $\mathrm{H}$ & -4.81095 & -1.54082 & 2.6829 \\
\hline $\mathrm{H}$ & $-0.32242-0.65083$ & -2.77682 & $\mathrm{H}$ & -4.33721 & -3.23814 & 2.78061 \\
\hline $\mathrm{C}$ & $-1.49502-0.15186$ & 5.1724 & $\mathrm{H}$ & -4.30458 & -2.22887 & 4.22864 \\
\hline $\mathrm{H}$ & $-0.43025-0.41891$ & 5.14642 & $\mathrm{C}$ & -2.25282 & -1.10882 & 6.10434 \\
\hline $\mathrm{H}$ & $\begin{array}{ll}-1.53728 & 0.87397\end{array}$ & 5.56231 & $\mathrm{H}$ & -2.18923 & -2.14192 & 5.74657 \\
\hline $\mathrm{C}$ & $-2.65516-1.84095$ & 2.83314 & $\mathrm{H}$ & -1.84664 & -1.0862 & 7.1205 \\
\hline $\mathrm{H}$ & $-2.48976-1.85899$ & 1.74971 & $\mathrm{H}$ & -3.31559 & -0.85244 & 6.17068 \\
\hline $\mathrm{H}$ & $-1.95762-2.57934$ & 3.25306 & $\mathrm{C}$ & -4.5305 & 1.12254 & 4.33482 \\
\hline $\mathrm{C}$ & $\begin{array}{ll}-3.49991 & 1.13789\end{array}$ & 3.19417 & $\mathrm{H}$ & -4.05124 & 1.29133 & 5.30469 \\
\hline $\mathrm{H}$ & $-3.02709 \quad 2.12895$ & 3.14934 & $\mathrm{H}$ & -5.28431 & 1.90518 & 4.20255 \\
\hline $\mathrm{H}$ & $-4.00882 \quad 0.99472$ & 2.23151 & $\mathrm{H}$ & -5.05659 & 0.16453 & 4.39383 \\
\hline $\mathrm{C}$ & $0.51177 \quad 2.89821$ & 6.4756 & $\mathrm{C}$ & -2.67097 & 5.12905 & 6.36018 \\
\hline $\mathrm{H}$ & $0.47911 \quad 1.82902$ & 6.23404 & $\mathrm{H}$ & -2.38312 & 6.08918 & 5.9195 \\
\hline $\mathrm{H}$ & 1.570193 .18605 & 6.4288 & $\mathrm{H}$ & -3.75416 & 5.14993 & 6.51476 \\
\hline $\mathrm{C}$ & $\begin{array}{ll}-2.25576 & 3.95929\end{array}$ & 5.45507 & $\mathrm{H}$ & -2.19991 & 5.06523 & 7.34646 \\
\hline $\mathrm{H}$ & $-2.76131 \quad 4.05541$ & 4.48361 & $\mathrm{C}$ & -0.0406 & 3.13108 & 7.88974 \\
\hline $\mathrm{H}$ & $-2.60882 \quad 3.01017$ & 5.87975 & $\mathrm{H}$ & -1.08808 & 2.81783 & 7.96131 \\
\hline $\mathrm{C}$ & $0.35296 \quad 5.50886$ & 4.79788 & $\mathrm{H}$ & 0.52349 & 2.56691 & 8.63862 \\
\hline $\mathrm{H}$ & $1.24802 \quad 5.36347$ & 4.17785 & $\mathrm{H}$ & 0.00466 & 4.18809 & 8.17324 \\
\hline $\mathrm{H}$ & $-0.33783 \quad 6.11806$ & 4.19973 & $\mathrm{C}$ & 0.74274 & 6.26016 & 6.08189 \\
\hline $\mathrm{H}$ & $2.9055 \quad 1.11198$ & 0.62359 & $\mathrm{H}$ & 1.47666 & 5.69222 & 6.66314 \\
\hline $\mathrm{C}$ & $2.78217-3.96225$ & -3.14458 & $\mathrm{H}$ & 1.18575 & 7.23503 & 5.85667 \\
\hline $\mathrm{H}$ & $3.03778-4.07498$ & -4.20255 & $\mathrm{H}$ & -0.12217 & 6.43535 & 6.72964 \\
\hline $\mathrm{H}$ & $3.54-4.50024-$ & 2.56511 & & & & \\
\hline $\mathrm{H}$ & $1.82473-4.46916$ & -2.98142 & & & & \\
\hline $\mathrm{C}$ & $\begin{array}{lll}4.80949 & -3.26267\end{array}$ & -0.1677 & $\mathrm{Et}_{3} \mathrm{Si}^{-7-}{ }^{1} C_{4}$ & & & \\
\hline $\mathrm{H}$ & $\begin{array}{lll}5.67641 & -3.21029\end{array}$ & 0.49808 & $\mathrm{C}$ & -0.2953 & -0.22141 & -0.17015 \\
\hline $\mathrm{H}$ & $4.39303-4.27163$ & -0.08452 & $\mathrm{C}$ & 1.23289 & -0.15065 & -0.236 \\
\hline $\mathrm{H}$ & $\begin{array}{lll}5.17483 & -3.14317\end{array}$ & -1.19315 & $\mathrm{C}$ & 1.69986 & 1.2988 & -0.07148 \\
\hline $\mathrm{C}$ & $1.56246 \quad-4.7611$ & 0.19749 & $\mathrm{C}$ & 1.06782 & 1.8921 & 1.17829 \\
\hline $\mathrm{H}$ & $2.24388-4.5923$ & 1.03793 & $\mathrm{C}$ & -0.89284 & 0.52573 & 1.03756 \\
\hline $\mathrm{H}$ & $\begin{array}{ll}0.7751 & -5.43778\end{array}$ & 0.54451 & $\mathrm{H}$ & 2.79333 & 1.29518 & 0.06001 \\
\hline $\mathrm{H}$ & $2.12578-5.28614$ & -0.58122 & $\mathrm{H}$ & 1.53493 & -0.51256 & -1.22995 \\
\hline $\mathrm{C}$ & $-1.89816-1.2312$ & -4.15593 & $\mathrm{H}$ & -0.66657 & 0.2861 & -1.07464 \\
\hline $\mathrm{H}$ & $-2.66567-2.00276$ & -4.27904 & $\mathrm{H}$ & 1.33005 & 2.94908 & 1.26541 \\
\hline $\mathrm{H}$ & $-2.40401-0.26002$ & -4.1707 & $\mathrm{H}$ & -1.9619 & 0.67798 & 0.85399 \\
\hline $\mathrm{H}$ & $\begin{array}{lll}-1.246 & -1.2753\end{array}$ & -5.03382 & $\mathrm{C}$ & -0.80111 & -0.21278 & 2.3801 \\
\hline $\mathrm{C}$ & $-3.27444-3.87475$ & -2.00806 & $\mathrm{H}$ & -0.91068 & 0.54687 & 3.17002 \\
\hline $\mathrm{H}$ & $-2.42759-4.07753$ & -2.6727 & $\mathrm{H}$ & 0.16596 & -0.71214 & 2.50766 \\
\hline $\mathrm{H}$ & $-3.68345-4.83904$ & -1.69184 & $\mathrm{O}$ & 1.30659 & 2.0451 & -1.20839 \\
\hline $\mathrm{H}$ & $-4.04392-3.37175$ & -2.60367 & $\mathrm{O}$ & 1.84888 & -0.90919 & 0.77853 \\
\hline $\mathrm{C}$ & $-4.74606-0.49638$ & -2.09763 & $\mathrm{O}$ & -0.65082 & -1.58493 & -0.1564 \\
\hline $\mathrm{H}$ & $-5.19244-1.36112$ & -1.59532 & $\mathrm{O}$ & -1.84728 & -1.16638 & 2.43511 \\
\hline $\mathrm{H}$ & $\begin{array}{ll}-5.50084 & 0.29562\end{array}$ & -2.11982 & $\mathrm{O}$ & -0.34987 & 7.84051 & 1.11281 \\
\hline $\mathrm{H}$ & $-4.54503-0.78731$ & -3.13347 & $\mathrm{Si}$ & 2.42856 & 2.50987 & -2.37908 \\
\hline
\end{tabular}




\begin{tabular}{|c|c|c|c|c|c|c|c|}
\hline $\mathrm{Si}$ & 2.10446 & -2.57851 & 0.8138 & $\mathrm{H}$ & 5.10819 & 0.32781 & -4.09941 \\
\hline $\mathrm{Si}$ & -1.98747 & -2.21299 & -0.96471 & $\mathrm{H}$ & 4.48436 & 1.89129 & -4.62855 \\
\hline $\mathrm{Si}$ & -2.54016 & -1.67729 & 3.87656 & $\mathrm{C}$ & 2.02365 & 3.36351 & -5.10638 \\
\hline $\mathrm{C}$ & 3.32347 & 0.97207 & -3.00929 & $\mathrm{H}$ & 2.22394 & 2.35137 & -5.47316 \\
\hline $\mathrm{H}$ & 3.53425 & 0.32728 & -2.14419 & $\mathrm{H}$ & 1.36705 & 3.85202 & -5.83262 \\
\hline $\mathrm{H}$ & 2.63566 & 0.40252 & -3.64923 & $\mathrm{H}$ & 2.97422 & 3.90692 & -5.10647 \\
\hline $\mathrm{C}$ & 3.65613 & 3.70894 & -1.59407 & $\mathrm{C}$ & 4.25976 & -4.01019 & 2.1652 \\
\hline $\mathrm{H}$ & 3.10989 & 4.27672 & -0.82917 & $\mathrm{H}$ & 5.29775 & -3.98967 & 2.51238 \\
\hline $\mathrm{H}$ & 4.42903 & 3.14099 & -1.0592 & $\mathrm{H}$ & 3.62667 & -4.19908 & 3.03858 \\
\hline $\mathrm{C}$ & 1.3856 & 3.32923 & -3.70921 & $\mathrm{H}$ & 4.15575 & -4.86917 & 1.49416 \\
\hline $\mathrm{H}$ & 1.13357 & 4.34583 & -3.38076 & $\mathrm{C}$ & 0.81547 & -4.91189 & 1.94679 \\
\hline $\mathrm{H}$ & 0.43699 & 2.77875 & -3.75009 & $\mathrm{H}$ & 1.76974 & -5.39276 & 2.18352 \\
\hline $\mathrm{C}$ & 0.90554 & -3.3792 & 2.02541 & $\mathrm{H}$ & 0.06749 & -5.30169 & 2.64595 \\
\hline $\mathrm{H}$ & 1.21202 & -3.08071 & 3.03833 & $\mathrm{H}$ & 0.52666 & -5.24476 & 0.94424 \\
\hline $\mathrm{H}$ & -0.08223 & -2.93228 & 1.8506 & $\mathrm{C}$ & 2.83974 & -4.59312 & -1.09939 \\
\hline $\mathrm{C}$ & 3.8693 & -2.69443 & 1.47742 & $\mathrm{H}$ & 2.73901 & -4.99806 & -2.11115 \\
\hline $\mathrm{H}$ & 4.56142 & -2.47552 & 0.65252 & $\mathrm{H}$ & 3.90245 & -4.3911 & -0.92973 \\
\hline $\mathrm{H}$ & 3.98104 & -1.86491 & 2.18851 & $\mathrm{H}$ & 2.54188 & -5.38134 & -0.39985 \\
\hline $\mathrm{C}$ & 1.9963 & -3.3204 & -0.91834 & $\mathrm{C}$ & -2.77881 & -4.95999 & -1.35579 \\
\hline $\mathrm{H}$ & 0.94431 & -3.5194 & -1.1557 & $\mathrm{H}$ & -2.26936 & -4.98075 & -2.32512 \\
\hline $\mathrm{H}$ & 2.32957 & -2.56299 & -1.64123 & $\mathrm{H}$ & -2.78849 & -5.98323 & -0.96796 \\
\hline $\mathrm{C}$ & -2.07821 & -3.99732 & -0.38502 & $\mathrm{H}$ & -3.81825 & -4.66896 & -1.53926 \\
\hline $\mathrm{H}$ & -2.55965 & -4.02439 & 0.60188 & $\mathrm{C}$ & -4.85348 & -2.03454 & -0.76269 \\
\hline $\mathrm{H}$ & -1.04451 & -4.32619 & -0.22122 & $\mathrm{H}$ & -4.88006 & -2.95426 & -0.16782 \\
\hline $\mathrm{C}$ & -3.54912 & -1.25829 & -0.51473 & $\mathrm{H}$ & -5.72971 & -1.44057 & -0.48481 \\
\hline $\mathrm{H}$ & -3.46508 & -1.01742 & 0.55244 & $\mathrm{H}$ & -4.97105 & -2.32126 & -1.81268 \\
\hline $\mathrm{H}$ & -3.56818 & -0.30152 & -1.05509 & $\mathrm{C}$ & -2.83127 & -2.16986 & -3.74364 \\
\hline $\mathrm{C}$ & -1.61223 & -2.10107 & -2.81212 & $\mathrm{H}$ & -3.52759 & -1.34943 & -3.54048 \\
\hline $\mathrm{H}$ & -1.07499 & -1.15727 & -2.98197 & $\mathrm{H}$ & -2.53884 & -2.1003 & -4.79599 \\
\hline $\mathrm{H}$ & -0.89747 & -2.89776 & -3.0606 & $\mathrm{H}$ & -3.38403 & -3.10699 & -3.61833 \\
\hline $\mathrm{C}$ & -3.67469 & -0.31179 & 4.51312 & $\mathrm{C}$ & -4.64862 & -3.60174 & 4.33396 \\
\hline $\mathrm{H}$ & -3.18061 & 0.6484 & 4.31078 & $\mathrm{H}$ & -5.38463 & -2.79523 & 4.41834 \\
\hline $\mathrm{H}$ & -4.58864 & -0.3131 & 3.90395 & $\mathrm{H}$ & -5.17701 & -4.49298 & 3.98203 \\
\hline $\mathrm{C}$ & -1.17962 & -2.05338 & 5.12709 & $\mathrm{H}$ & -4.28008 & -3.8155 & 5.34257 \\
\hline $\mathrm{H}$ & -0.31812 & -2.43281 & 4.5628 & $\mathrm{C}$ & -1.57134 & -3.07401 & 6.20786 \\
\hline $\mathrm{H}$ & -0.84996 & -1.11546 & 5.59414 & $\mathrm{H}$ & -1.83915 & -4.03656 & 5.75916 \\
\hline $\mathrm{C}$ & -3.50911 & -3.20958 & 3.38161 & $\mathrm{H}$ & -0.74837 & -3.25459 & 6.90621 \\
\hline $\mathrm{H}$ & -3.91762 & -3.0048 & 2.38193 & $\mathrm{H}$ & -2.43146 & -2.73548 & 6.79493 \\
\hline $\mathrm{H}$ & -2.80609 & -4.04482 & 3.25693 & $\mathrm{C}$ & -4.02683 & -0.40246 & 6.00582 \\
\hline $\mathrm{H}$ & 1.44048 & 1.35725 & 2.06283 & $\mathrm{H}$ & -3.1263 & -0.34668 & 6.62653 \\
\hline $\mathrm{C}$ & 4.31262 & 4.68301 & -2.58614 & $\mathrm{H}$ & -4.68917 & 0.41237 & 6.31406 \\
\hline $\mathrm{H}$ & 3.56007 & 5.2985 & -3.09002 & $\mathrm{H}$ & -4.53172 & -1.34405 & 6.24644 \\
\hline $\mathrm{H}$ & 5.0078 & 5.36087 & -2.08157 & & & & \\
\hline $\mathrm{H}$ & 4.87583 & 4.154 & -3.36189 & $\mathrm{Me}_{3} \mathrm{Si}-8-{ }^{4} C_{1}$ & & & \\
\hline $\mathrm{C}$ & 4.6392 & 1.25349 & -3.75242 & $\mathrm{C}$ & -0.99832 & -0.38121 & -0.20157 \\
\hline $\mathrm{H}$ & 5.35733 & 1.76068 & -3.09967 & $\mathrm{C}$ & 0.50616 & -0.49719 & -0.44595 \\
\hline
\end{tabular}




\begin{tabular}{|c|c|c|c|c|c|c|}
\hline $\mathrm{C}$ & 1.1109 & $0.90158-0.36712$ & $\mathrm{H}$ & -5.07055 & $5 \quad 1.88304$ & 2.52936 \\
\hline $\mathrm{C}$ & -0.53567 & $\begin{array}{lll}7.68705 & 1.13917\end{array}$ & $\mathrm{H}$ & -5.65814 & $4 \quad 0.65573$ & 1.39141 \\
\hline $\mathrm{C}$ & -1.26597 & $\begin{array}{lll}7 & 0.33471 & 1.11976\end{array}$ & $\mathrm{C}$ & -2.75414 & 0.21629 & 4.16492 \\
\hline $\mathrm{H}$ & 0.673 & $-0.90344-1.45571$ & $\mathrm{H}$ & -3.40586 & $5-0.14785$ & 4.96693 \\
\hline $\mathrm{H}$ & -1.41605 & $5 \quad 0.25448-1.00244$ & $\mathrm{H}$ & -1.79434 & $4-0.30365$ & 4.25639 \\
\hline $\mathrm{H}$ & -0.97614 & $\begin{array}{lll}4 & 2.33252 & 0.35921\end{array}$ & $\mathrm{H}$ & -2.58568 & 1.28694 & 4.30952 \\
\hline $\mathrm{H}$ & -0.86422 & $\begin{array}{lll}2 & -0.29205 & 1.93194\end{array}$ & $\mathrm{C}$ & -3.7945 & -1.94001 & 2.27636 \\
\hline $\mathrm{H}$ & 0.70473 & $\begin{array}{ll}1.5265 & -1.17816\end{array}$ & $\mathrm{H}$ & -4.41283 & $3-2.1619$ & 1.40098 \\
\hline $\mathrm{C}$ & -0.60313 & $2.42043 \quad 2.47449$ & $\mathrm{H}$ & -2.82463 & $3-2.42738$ & 2.13182 \\
\hline $\mathrm{H}$ & 0.19066 & $3.18185 \quad 2.45823$ & $\mathrm{H}$ & -4.27923 & $3-2.37744$ & 3.15617 \\
\hline $\mathrm{H}$ & -0.3763 & $1.71658 \quad 3.29056$ & $\mathrm{C}$ & -1.37868 & 5.84514 & 3.03438 \\
\hline $\mathrm{O}$ & 1.05077 & $\begin{array}{ll}-1.35785 & 0.53031\end{array}$ & $\mathrm{H}$ & -1.49114 & $4 \quad 6.67511$ & 3.7401 \\
\hline $\mathrm{O}$ & -1.61233 & $3-1.6488-0.18825$ & $\mathrm{H}$ & -0.31225 & $5 \quad 5.7424$ & 2.80762 \\
\hline $\mathrm{O}$ & -2.65175 & $5 \quad 0.54862 \quad 1.25372$ & $\mathrm{H}$ & -1.90129 & 6.10956 & 2.10992 \\
\hline $\mathrm{O}$ & -1.8728 & $3.0067 \quad 2.66896$ & $\mathrm{C}$ & -1.16045 & 3.85656 & 5.35765 \\
\hline $\mathrm{O}$ & 0.85109 & 1.500920 .88872 & $\mathrm{H}$ & -1.31119 & 4.65561 & 6.09141 \\
\hline $\mathrm{Si}$ & 2.61516 & -1.972210 .52388 & $\mathrm{H}$ & -1.51602 & 2.92053 & 5.8008 \\
\hline $\mathrm{Si}$ & -2.63786 & $-2.13323-1.42947$ & $\mathrm{H}$ & -0.08298 & 3.7642 & 5.184 \\
\hline $\mathrm{Si}$ & -3.57362 & $-0.09038 \quad 2.50417$ & $\mathrm{C}$ & -3.91803 & 4.38057 & 4.03883 \\
\hline $\mathrm{Si}$ & -2.07326 & $4.2651 \quad 3.76846$ & $\mathrm{H}$ & -4.44019 & 4.5303 & 3.08841 \\
\hline $\mathrm{C}$ & 2.49444 & $-3.62432 \quad 1.39093$ & $\mathrm{H}$ & -4.29992 & 23.46354 & 4.4998 \\
\hline $\mathrm{H}$ & 1.84854 & $-4.30974 \quad 0.83343$ & $\mathrm{H}$ & -4.16174 & $4 \quad 5.22049$ & 4.69724 \\
\hline $\mathrm{H}$ & 3.48278 & $-4.08507 \quad 1.48857$ & $\mathrm{H}$ & 2.19812 & 0.86347 & -0.47536 \\
\hline $\mathrm{H}$ & 2.0766 & -3.501412 .3952 & & & & \\
\hline $\mathrm{C}$ & 3.7618 & $-0.83375 \quad 1.47801$ & $\mathrm{Me}_{3} \mathrm{Si}_{-}-{ }^{-1}{ }^{1} C_{4}$ & & & \\
\hline $\mathrm{H}$ & 4.74562 & $-1.30117 \quad 1.5953$ & $\mathrm{C}$ & 0.0336 & -0.10013 & 0.02989 \\
\hline $\mathrm{H}$ & 3.90467 & $0.12967 \quad 0.97921$ & $\mathrm{C}$ & 1.54766 & -0.16108 & 0.18557 \\
\hline $\mathrm{H}$ & 3.35739 & $-0.64159 \quad 2.47731$ & $\mathrm{C}$ & 1.58034 & 1.86587 & 1.4769 \\
\hline $\mathrm{C}$ & 3.19867 & $-2.16878-1.24917$ & $\mathrm{C}$ & 0.04605 & 1.90923 & 1.50285 \\
\hline $\mathrm{H}$ & 4.18507 & $-2.64482-1.25943$ & $\mathrm{C}$ & -0.5763 & 0.52543 & 1.28027 \\
\hline $\mathrm{H}$ & 2.51368 & $-2.8015-1.82358$ & $\mathrm{H}$ & 2.00376 & -0.56502 & -0.7213 \\
\hline $\mathrm{H}$ & 3.29089 & $-1.20689-1.76488$ & $\mathrm{H}$ & -0.1922 & 0.56479 & -0.81741 \\
\hline $\mathrm{C}$ & -4.13414 & $-1.00406-1.51334$ & $\mathrm{H}$ & 1.92113 & 2.89853 & 1.32784 \\
\hline $\mathrm{H}$ & -4.69023 & $\begin{array}{lll}3 & -1.0089 & -0.57126\end{array}$ & $\mathrm{H}$ & -0.26406 & $5 \quad 2.26943$ & 2.49501 \\
\hline $\mathrm{H}$ & -3.84013 & $\begin{array}{lll}3 & 0.02963 & -1.7232\end{array}$ & $\mathrm{H}$ & -1.66025 & $5 \quad 0.65439$ & 1.1264 \\
\hline $\mathrm{H}$ & -4.80782 & $2-1.33148-2.31284$ & $\mathrm{C}$ & 2.20925 & 1.36172 & 2.77945 \\
\hline $\mathrm{C}$ & -3.10355 & $-3.89612-1.02137$ & $\mathrm{H}$ & 3.30109 & 1.3784 & 2.64911 \\
\hline $\mathrm{H}$ & -2.21342 & $2-4.53319-1.01311$ & $\mathrm{H}$ & 1.90367 & 0.33622 & 2.99865 \\
\hline $\mathrm{H}$ & -3.58153 & $\begin{array}{lll}3 & -3.9637 & -0.0397\end{array}$ & $\mathrm{O}$ & -0.47606 & $5-1.40189$ & -0.17187 \\
\hline $\mathrm{H}$ & -3.79877 & $7 \quad-4.29208-1.7687$ & $\mathrm{O}$ & -0.30107 & $7 \quad-0.29455$ & 2.39702 \\
\hline $\mathrm{C}$ & -1.70408 & $-2.04676-3.05762$ & $\mathrm{O}$ & -0.41687 & $7 \quad 2.76058$ & 0.46459 \\
\hline $\mathrm{H}$ & -0.81549 & $-2.68637-3.03201$ & $\mathrm{O}$ & 1.78858 & 2.19672 & 3.84778 \\
\hline $\mathrm{H}$ & -2.34142 & $2-2.38246-3.88274$ & $\mathrm{O}$ & 2.08022 & 1.14929 & 0.35256 \\
\hline $\mathrm{H}$ & -1.38247 & $7 \quad-1.02368-3.28291$ & $\mathrm{Si}$ & -1.5401 & -1.70492 & -1.44301 \\
\hline $\mathrm{C}$ & -5.20863 & $\begin{array}{lll}3 & 0.80773 & 2.3782\end{array}$ & $\mathrm{Si}$ & -1.50833 & -0.83578 & 3.43512 \\
\hline $\mathrm{H}$ & -5.9127 & $0.44186 \quad 3.13263$ & $\mathrm{Si}$ & -1.09441 & 4.25704 & 0.8407 \\
\hline
\end{tabular}




\begin{tabular}{|c|c|c|c|c|c|c|c|}
\hline $\mathrm{Si}$ & 2.86463 & 3.23621 & 4.62028 & $\mathrm{H}$ & 3.67461 & 1.57057 & 6.27678 \\
\hline $\mathrm{C}$ & -2.13626 & -3.45571 & -1.18735 & $\mathrm{H}$ & 4.73749 & 1.60682 & 4.85748 \\
\hline $\mathrm{H}$ & -2.78339 & -3.76414 & -2.01473 & $\mathrm{H}$ & 4.84345 & 2.88829 & 6.07219 \\
\hline $\mathrm{H}$ & -1.29119 & -4.14942 & -1.13905 & $\mathrm{H}$ & 1.80422 & -0.8129 & 1.03072 \\
\hline $\mathrm{H}$ & -2.70611 & -3.54178 & -0.25721 & & & & \\
\hline $\mathrm{C}$ & -0.61304 & -1.51931 & -3.06291 & $\mathrm{Et}_{3} \mathrm{Si}-8-{ }^{4} C_{1}$ & & & \\
\hline $\mathrm{H}$ & -0.20323 & -0.50944 & -3.17322 & $\mathrm{C}$ & -0.98951 & -0.44319 & -0.30262 \\
\hline $\mathrm{H}$ & 0.21632 & -2.23151 & -3.1189 & $\mathrm{C}$ & 0.50737 & -0.60809 & -0.57189 \\
\hline $\mathrm{H}$ & -1.27973 & -1.70352 & -3.9119 & $\mathrm{C}$ & 1.16254 & 0.76783 & -0.49197 \\
\hline $\mathrm{C}$ & -2.95676 & -0.47259 & -1.38609 & $\mathrm{C}$ & -0.43369 & 1.59972 & 1.03994 \\
\hline $\mathrm{H}$ & -3.53893 & -0.56789 & -0.46381 & $\mathrm{C}$ & -1.20558 & 0.26862 & 1.0355 \\
\hline $\mathrm{H}$ & -2.59573 & 0.55977 & -1.45542 & $\mathrm{H}$ & 0.64599 & -1.00875 & -1.58779 \\
\hline $\mathrm{H}$ & -3.63383 & -0.64391 & -2.22994 & $\mathrm{H}$ & -1.40339 & 0.21286 & -1.08928 \\
\hline $\mathrm{C}$ & -0.58668 & -1.64307 & 4.84481 & $\mathrm{H}$ & -0.86105 & 2.25556 & 0.26164 \\
\hline $\mathrm{H}$ & 0.02906 & -2.47132 & 4.48063 & $\mathrm{H}$ & -0.7991 & -0.37233 & 1.8347 \\
\hline $\mathrm{H}$ & 0.06875 & -0.91889 & 5.33927 & $\mathrm{H}$ & 0.76446 & 1.41214 & -1.29157 \\
\hline $\mathrm{H}$ & -1.28376 & -2.03854 & 5.59033 & $\mathrm{C}$ & -0.46206 & 2.34756 & 2.36928 \\
\hline $\mathrm{C}$ & -2.61014 & -2.05484 & 2.53439 & $\mathrm{H}$ & 0.4034 & 3.02607 & 2.37335 \\
\hline $\mathrm{H}$ & -3.05479 & -1.59732 & 1.64388 & $\mathrm{H}$ & -0.33231 & 1.63084 & 3.19563 \\
\hline $\mathrm{H}$ & -2.03265 & -2.92671 & 2.21163 & $\mathrm{O}$ & 1.02648 & -1.50404 & 0.38592 \\
\hline $\mathrm{H}$ & -3.42462 & -2.39685 & 3.18171 & $\mathrm{O}$ & -1.63516 & - -1.69364 & -0.29587 \\
\hline $\mathrm{C}$ & -2.51279 & 0.63836 & 4.01799 & $\mathrm{O}$ & -2.58177 & 0.51617 & 1.20896 \\
\hline $\mathrm{H}$ & -3.2729 & 0.32221 & 4.7403 & $\mathrm{O}$ & -1.67002 & 3.06137 & 2.51365 \\
\hline $\mathrm{H}$ & -1.86966 & 1.38036 & 4.50407 & $\mathrm{O}$ & 0.94263 & 1.36467 & 0.77162 \\
\hline $\mathrm{H}$ & -3.03047 & 1.12621 & 3.18473 & $\mathrm{Si}$ & 2.62282 & -2.0234 & 0.50297 \\
\hline $\mathrm{C}$ & 0.15381 & 5.24686 & 1.83226 & $\mathrm{Si}$ & -2.79506 & -2.12808 & -1.43541 \\
\hline $\mathrm{H}$ & 0.47745 & 4.68193 & 2.71515 & $\mathrm{Si}$ & -3.52921 & -0.16743 & 2.42263 \\
\hline $\mathrm{H}$ & 1.03971 & 5.4764 & 1.23072 & $\mathrm{Si}$ & -1.88911 & 4.19929 & 3.73763 \\
\hline $\mathrm{H}$ & -0.2796 & 6.19085 & 2.17859 & $\mathrm{C}$ & 2.49672 & -3.71339 & 1.31932 \\
\hline $\mathrm{C}$ & -2.64546 & 3.98663 & 1.86011 & $\mathrm{H}$ & 2.18358 & -4.44748 & 0.56519 \\
\hline $\mathrm{H}$ & -3.33192 & 3.30166 & 1.35095 & $\mathrm{H}$ & 1.67256 & -3.65083 & 2.04207 \\
\hline $\mathrm{H}$ & -2.4113 & 3.56842 & 2.84488 & $\mathrm{C}$ & 3.59148 & -0.82752 & 1.59659 \\
\hline $\mathrm{H}$ & -3.16669 & 4.93673 & 2.01886 & $\mathrm{H}$ & 3.20642 & 0.18391 & 1.41714 \\
\hline $\mathrm{C}$ & -1.47876 & 5.04652 & -0.80658 & $\mathrm{H}$ & 3.34764 & -1.05506 & 2.64339 \\
\hline $\mathrm{H}$ & -1.92172 & 6.03743 & -0.6652 & $\mathrm{C}$ & 3.35871 & -2.1356 & -1.23407 \\
\hline $\mathrm{H}$ & -0.56877 & 5.16103 & -1.4033 & $\mathrm{H}$ & 2.55148 & -2.43494 & -1.91615 \\
\hline $\mathrm{H}$ & -2.18723 & 4.43441 & -1.37334 & $\mathrm{H}$ & 3.68648 & -1.14146 & -1.56668 \\
\hline $\mathrm{C}$ & 1.80994 & 4.23498 & 5.7953 & $\mathrm{C}$ & -4.31153 & -1.02107 & -1.2472 \\
\hline $\mathrm{H}$ & 1.29389 & 3.5798 & 6.50394 & $\mathrm{H}$ & -4.90127 & -1.36651 & -0.38741 \\
\hline $\mathrm{H}$ & 2.42875 & 4.93418 & 6.36682 & $\mathrm{H}$ & -3.94155 & -0.02569 & -0.96708 \\
\hline $\mathrm{H}$ & 1.05651 & 4.81422 & 5.25226 & $\mathrm{C}$ & -3.15978 & -3.93298 & -1.05459 \\
\hline $\mathrm{C}$ & 3.7086 & 4.32772 & 3.34765 & $\mathrm{H}$ & -2.38146 & -4.55229 & -1.51966 \\
\hline $\mathrm{H}$ & 4.44379 & 4.97531 & 3.83739 & $\mathrm{H}$ & -3.04718 & -4.06556 & 0.02904 \\
\hline $\mathrm{H}$ & 4.23947 & 3.73376 & 2.59558 & $\mathrm{C}$ & -2.05389 & -1.92495 & -3.16267 \\
\hline $\mathrm{H}$ & 2.98533 & 4.96664 & 2.83113 & $\mathrm{H}$ & -0.98732 & -2.17733 & -3.09263 \\
\hline $\mathrm{C}$ & 4.14961 & 2.22771 & 5.54156 & $\mathrm{H}$ & -2.10072 & -0.86833 & -3.45949 \\
\hline
\end{tabular}




\begin{tabular}{|c|c|c|c|c|c|c|c|}
\hline $\mathrm{C}$ & -5.23605 & 0.56208 & 2.11029 & $\mathrm{H}$ & -3.26011 & -2.52976 & 4.41645 \\
\hline $\mathrm{H}$ & -5.27422 & 1.55681 & 2.57517 & $\mathrm{C}$ & -6.40088 & -0.30451 & 2.61068 \\
\hline $\mathrm{H}$ & -5.32954 & 0.72645 & 1.02966 & $\mathrm{H}$ & -7.36899 & 0.16229 & 2.40437 \\
\hline $\mathrm{C}$ & -2.87653 & 0.38111 & 4.10653 & $\mathrm{H}$ & -6.39832 & -1.28732 & 2.12554 \\
\hline $\mathrm{H}$ & -1.99806 & -0.21658 & 4.38741 & $\mathrm{H}$ & -6.34188 & -0.47465 & 3.69109 \\
\hline $\mathrm{H}$ & -2.5205 & 1.40551 & 3.951 & $\mathrm{C}$ & -3.91027 & 0.37341 & 5.24375 \\
\hline $\mathrm{C}$ & -3.49919 & -2.05082 & 2.2999 & $\mathrm{H}$ & -3.48487 & 0.77172 & 6.17137 \\
\hline $\mathrm{H}$ & -4.12973 & -2.36498 & 1.45768 & $\mathrm{H}$ & -4.77883 & 0.99193 & 4.99112 \\
\hline $\mathrm{H}$ & -2.47803 & -2.34126 & 2.02167 & $\mathrm{H}$ & -4.2798 & -0.6346 & 5.45734 \\
\hline $\mathrm{C}$ & -1.13679 & 5.82961 & 3.16407 & $\mathrm{C}$ & -1.65328 & 4.16974 & 6.60467 \\
\hline $\mathrm{H}$ & -0.21248 & 5.58659 & 2.62227 & $\mathrm{H}$ & -1.13241 & 3.79929 & 7.49292 \\
\hline $\mathrm{H}$ & -1.8117 & 6.27651 & 2.42191 & $\mathrm{H}$ & -1.5998 & 5.26316 & 6.62558 \\
\hline $\mathrm{C}$ & -1.04764 & 3.59039 & 5.31561 & $\mathrm{H}$ & -2.70824 & 3.89103 & 6.70109 \\
\hline $\mathrm{H}$ & -1.12104 & 2.49507 & 5.34623 & $\mathrm{C}$ & -4.27413 & 5.61889 & 4.53491 \\
\hline $\mathrm{H}$ & 0.02507 & 3.82126 & 5.26458 & $\mathrm{H}$ & -5.36548 & 5.62114 & 4.61448 \\
\hline $\mathrm{C}$ & -3.7563 & 4.30599 & 3.92885 & $\mathrm{H}$ & -3.87138 & 5.78845 & 5.53884 \\
\hline $\mathrm{H}$ & -4.18338 & 4.16539 & 2.9271 & $\mathrm{H}$ & -3.98723 & 6.47699 & 3.91797 \\
\hline $\mathrm{H}$ & -4.09641 & 3.44808 & 4.52574 & $\mathrm{C}$ & -0.82626 & 6.83895 & 4.27967 \\
\hline $\mathrm{H}$ & 2.24656 & 0.69696 & -0.62106 & $\mathrm{H}$ & -0.11183 & 6.42472 & 4.99902 \\
\hline $\mathrm{C}$ & 5.11352 & -0.85392 & 1.38951 & $\mathrm{H}$ & -0.39135 & 7.75939 & 3.878 \\
\hline $\mathrm{H}$ & 5.62095 & -0.14246 & 2.04851 & $\mathrm{H}$ & -1.727 & 7.11871 & 4.83616 \\
\hline $\mathrm{H}$ & $5.534-$ & -1.84478 & 1.59206 & & & & \\
\hline $\mathrm{H}$ & 5.3755 & -0.59219 & 0.35851 & $\mathrm{Et}_{3} \mathrm{Si}-8-{ }^{1} C_{4}$ & & & \\
\hline $\mathrm{C}$ & 3.77293 & -4.18619 & 2.03084 & $\mathrm{C}$ & 0.04811 & -0.13635 & -0.07144 \\
\hline $\mathrm{H}$ & 4.07053 & -3.48209 & 2.81513 & $\mathrm{C}$ & 1.56597 & -0.21525 & 0.03852 \\
\hline $\mathrm{H}$ & 3.63034 & -5.16302 & 2.50301 & $\mathrm{C}$ & 1.6676 & 1.80974 & 1.33081 \\
\hline $\mathrm{H}$ & 4.61509 & -4.27746 & 1.33701 & $\mathrm{C}$ & 0.13566 & 1.8764 & 1.40449 \\
\hline $\mathrm{C}$ & 4.51864 & -3.13709 & -1.35822 & $\mathrm{C}$ & -0.51364 & 0.50384 & 1.19536 \\
\hline $\mathrm{H}$ & 4.19468 & -4.14779 & -1.08904 & $\mathrm{H}$ & 1.98923 & -0.62258 & -0.8828 \\
\hline $\mathrm{H}$ & 4.90521 & -3.17644 & -2.38119 & $\mathrm{H}$ & -0.20372 & 0.52248 & -0.91697 \\
\hline $\mathrm{H}$ & 5.3543 & -2.87491 & -0.70135 & $\mathrm{H}$ & 2.01247 & 2.83626 & 1.15916 \\
\hline $\mathrm{C}$ & -2.71344 & -2.80382 & -4.23811 & $\mathrm{H}$ & -0.13641 & 2.22843 & 2.41017 \\
\hline $\mathrm{H}$ & -2.24478 & -2.6608 & -5.21666 & $\mathrm{H}$ & -1.5996 & 0.65012 & 1.0763 \\
\hline $\mathrm{H}$ & -2.62791 & -3.8659 & -3.98456 & $\mathrm{C}$ & 2.32735 & 1.30804 & 2.62029 \\
\hline $\mathrm{H}$ & -3.77915 & -2.57947 & -4.34928 & $\mathrm{H}$ & 3.41677 & 1.3947 & 2.4981 \\
\hline $\mathrm{C}$ & -5.21105 & -0.91986 & -2.48802 & $\mathrm{H}$ & 2.08072 & 0.25957 & 2.80576 \\
\hline $\mathrm{H}$ & -6.05837 & -0.24962 & -2.31115 & $\mathrm{O}$ & -0.47036 & $5-1.4389$ & -0.24364 \\
\hline $\mathrm{H}$ & -4.65781 & -0.52907 & -3.34874 & $\mathrm{O}$ & -0.21627 & -0.31852 & 2.30484 \\
\hline $\mathrm{H}$ & -5.61949 & -1.8949 & -2.77494 & $\mathrm{O}$ & -0.34251 & 2.75438 & 0.39531 \\
\hline $\mathrm{C}$ & -4.5521 & -4.40658 & -1.49817 & $\mathrm{O}$ & 1.85161 & 2.08345 & 3.70822 \\
\hline $\mathrm{H}$ & -4.71508 & -5.46089 & -1.25461 & $\mathrm{O}$ & 2.12678 & 1.08341 & 0.19598 \\
\hline $\mathrm{H}$ & -5.34171 & -3.8289 & -1.00492 & $\mathrm{Si}$ & -1.54732 & -1.79108 & -1.493 \\
\hline $\mathrm{H}$ & -4.69206 & -4.29423 & -2.57875 & $\mathrm{Si}$ & -1.41217 & -0.82423 & 3.37778 \\
\hline $\mathrm{C}$ & -3.92044 & -2.78579 & 3.58117 & $\mathrm{Si}$ & -0.9358 & 4.27467 & 0.82635 \\
\hline $\mathrm{H}$ & -4.94261 & -2.53261 & 3.88222 & $\mathrm{Si}$ & 2.81052 & 3.16929 & 4.56562 \\
\hline $\mathrm{H}$ & -3.87725 & -3.87181 & 3.44958 & $\mathrm{C}$ & -2.1412 & -3.53764 & -1.1329 \\
\hline
\end{tabular}




\begin{tabular}{|c|c|c|c|c|c|c|c|}
\hline $\mathrm{H}$ & -1.30693 & -4.07062 & -0.65872 & $\mathrm{H}$ & -2.94384 & -5.32676 & -2.10308 \\
\hline $\mathrm{H}$ & -2.94053 & -3.49233 & -0.38125 & $\mathrm{H}$ & -3.46739 & -3.81903 & -2.85543 \\
\hline $\mathrm{C}$ & -0.5992 & -1.67818 & -3.11892 & $\mathrm{C}$ & -3.49767 & -2.81989 & 3.22145 \\
\hline $\mathrm{H}$ & 0.09676 & -0.83217 & -3.03136 & $\mathrm{H}$ & -4.04809 & -2.36903 & 4.05412 \\
\hline $\mathrm{H}$ & 0.02946 & -2.57364 & -3.21304 & $\mathrm{H}$ & -4.22369 & -3.35227 & 2.59879 \\
\hline $\mathrm{C}$ & -2.96836 & -0.54912 & -1.45282 & $\mathrm{H}$ & -2.8174 & -3.56648 & 3.6447 \\
\hline $\mathrm{H}$ & -3.1742 & -0.32017 & -0.39881 & $\mathrm{C}$ & -3.52009 & 0.4776 & 4.86529 \\
\hline $\mathrm{H}$ & -2.64579 & 0.39457 & -1.91345 & $\mathrm{H}$ & -3.90362 & 1.39838 & 5.31557 \\
\hline $\mathrm{C}$ & -0.48883 & -1.91417 & 4.59683 & $\mathrm{H}$ & -4.26171 & 0.13511 & 4.13581 \\
\hline $\mathrm{H}$ & -0.31337 & -2.89304 & 4.13221 & $\mathrm{H}$ & -3.46639 & -0.27733 & 5.65651 \\
\hline $\mathrm{H}$ & 0.50137 & -1.46 & 4.73488 & $\mathrm{C}$ & -1.17537 & -2.08477 & 5.96047 \\
\hline $\mathrm{C}$ & -2.72745 & -1.76666 & 2.4098 & $\mathrm{H}$ & -0.59044 & -2.72769 & 6.62508 \\
\hline $\mathrm{H}$ & -3.43139 & -1.05154 & 1.96166 & $\mathrm{H}$ & -1.29926 & -1.11938 & 6.46221 \\
\hline $\mathrm{H}$ & -2.20347 & -2.24434 & 1.57146 & $\mathrm{H}$ & -2.16942 & -2.53337 & 5.8616 \\
\hline $\mathrm{C}$ & -2.15474 & 0.70219 & 4.19732 & $\mathrm{C}$ & -3.46524 & 5.20482 & 1.85502 \\
\hline $\mathrm{H}$ & -1.43046 & 1.10227 & 4.92001 & $\mathrm{H}$ & -3.83087 & 5.36493 & 0.83538 \\
\hline $\mathrm{H}$ & -2.25841 & 1.47132 & 3.41897 & $\mathrm{H}$ & -4.33498 & 5.01575 & 2.49142 \\
\hline $\mathrm{C}$ & 0.40085 & 5.18372 & 1.80108 & $\mathrm{H}$ & -3.01072 & 6.14269 & 2.19099 \\
\hline $\mathrm{H}$ & 0.85386 & 4.44927 & 2.48395 & $\mathrm{C}$ & -1.37694 & 6.63433 & -0.77171 \\
\hline $\mathrm{H}$ & 1.20053 & 5.49178 & 1.11338 & $\mathrm{H}$ & -1.61096 & 7.05295 & -1.75509 \\
\hline $\mathrm{C}$ & -2.46514 & 4.03808 & 1.90757 & $\mathrm{H}$ & -2.13245 & 7.00314 & -0.07018 \\
\hline $\mathrm{H}$ & -2.96092 & 3.11409 & 1.57838 & $\mathrm{H}$ & -0.41088 & 7.04542 & -0.46075 \\
\hline $\mathrm{H}$ & -2.14965 & 3.86403 & 2.94574 & $\mathrm{C}$ & -0.09671 & 6.38527 & 2.6197 \\
\hline $\mathrm{C}$ & -1.33609 & 5.09893 & -0.81237 & $\mathrm{H}$ & -0.83513 & 6.07324 & 3.36629 \\
\hline $\mathrm{H}$ & -0.57234 & 4.76869 & -1.52773 & $\mathrm{H}$ & 0.72489 & 6.87244 & 3.15538 \\
\hline $\mathrm{H}$ & -2.2909 & 4.70208 & -1.18102 & $\mathrm{H}$ & -0.56915 & 7.14367 & 1.98715 \\
\hline $\mathrm{C}$ & 1.56034 & 4.22831 & 5.48894 & $\mathrm{C}$ & 2.08779 & 4.90474 & 6.76318 \\
\hline $\mathrm{H}$ & 0.71847 & 3.56906 & 5.73943 & $\mathrm{H}$ & 1.30504 & 5.48565 & 7.26028 \\
\hline $\mathrm{H}$ & 1.15768 & 4.98267 & 4.79989 & $\mathrm{H}$ & 2.45388 & 4.16356 & 7.48132 \\
\hline $\mathrm{C}$ & 3.85898 & 4.17833 & 3.36019 & $\mathrm{H}$ & 2.91505 & 5.58832 & 6.54576 \\
\hline $\mathrm{H}$ & 4.77598 & 3.62271 & 3.1233 & $\mathrm{C}$ & 4.21427 & 5.58138 & 3.87991 \\
\hline $\mathrm{H}$ & 3.30755 & 4.27415 & 2.41595 & $\mathrm{H}$ & 4.81805 & 6.13605 & 3.15535 \\
\hline $\mathrm{C}$ & 3.92426 & 2.19909 & 5.73555 & $\mathrm{H}$ & 3.31028 & 6.16875 & 4.07504 \\
\hline $\mathrm{H}$ & 3.31419 & 1.82274 & 6.56727 & $\mathrm{H}$ & 4.78347 & 5.53535 & 4.81442 \\
\hline $\mathrm{H}$ & 4.27072 & 1.31079 & 5.18952 & $\mathrm{C}$ & 5.13602 & 2.97822 & 6.26979 \\
\hline $\mathrm{H}$ & 1.83463 & -0.8767 & 0.8722 & $\mathrm{H}$ & 5.7456 & 2.36389 & 6.93945 \\
\hline $\mathrm{C}$ & -1.46375 & -1.4974 & -4.37577 & $\mathrm{H}$ & 5.78242 & 3.3116 & 5.45109 \\
\hline $\mathrm{H}$ & -0.84993 & -1.43915 & -5.27979 & $\mathrm{H}$ & 4.83087 & 3.8687 & 6.8291 \\
\hline $\mathrm{H}$ & -2.16497 & -2.32787 & -4.50852 & & & & \\
\hline $\mathrm{H}$ & -2.05284 & -0.57608 & -4.31876 & & & & \\
\hline $\mathrm{C}$ & -4.25924 & -1.05097 & -2.12102 & & & & \\
\hline $\mathrm{H}$ & -5.05273 & -0.29911 & -2.07309 & & & & \\
\hline $\mathrm{H}$ & -4.10316 & -1.29619 & -3.17648 & & & & \\
\hline $\mathrm{H}$ & -4.63148 & -1.95417 & -1.62592 & & & & \\
\hline $\mathrm{C}$ & -2.62152 & -4.3154 & -2.36824 & & & & \\
\hline $\mathrm{H}$ & -1.8234 & -4.40842 & -3.11209 & & & & \\
\hline
\end{tabular}

